\title{
Reducing the Cost of Ensemble-Based Data Assimilation in Multiple-Query Scenarios through Covariance Augmentation
}

by

\author{
Andrew F. Ilersich
}

A thesis submitted in conformity with the requirements for the degree of Master of Applied Science

Institute for Aerospace Studies

University of Toronto

(C) Copyright 2021 by Andrew F. Ilersich 


\begin{abstract}
Reducing the Cost of Ensemble-Based Data Assimilation in Multiple-Query Scenarios through Covariance Augmentation

Andrew F. Ilersich

Master of Applied Science

Institute for Aerospace Studies

University of Toronto

2021
\end{abstract}

We present and assess a method to reduce the computational cost of performing data assimilation (DA) for reacting flow in multiple-query scenarios, where we consider several scenarios with similar underlying dynamics. We focus on ensemble-based DA, in particular the ensemble Kalman filter (EnKF). The accuracy of the EnKF, which depends on the quality of the sample covariance, improves with the ensemble size, but so does its computational cost. To reduce the ensemble size while maintaining accurate covariance, we propose a data-driven approach to augment the covariance based on the statistical behavior learned from model evaluations. We assess our augmentation method using one-dimensional model problems and synthetic reacting flow cases. We show in all cases that ensemble size, and thus computational cost, may be reduced by a factor of three to four while maintaining accuracy. 


\section{Acknowledgements}

I am grateful to Professor Masayuki Yano and Professor Adam Steinberg for their supervision and mentorship over the past two years. I appreciate your endless enthusiasm, patience, and encouragement to play around with new methods and explore what might be possible. I especially appreciate the efforts you made to maintain all this despite the pandemic. My thesis would not be where it is without your guidance.

I would like to thank Professor Peter Grant for support in side projects and extracurriculars, especially in letting me use the aero design lab. You didn't have to do that, and I appreciate so much that you did. I would also like to thank Professor Gabriele D'Eleuterio for the nice chats and advice every time I passed by his office. I'm looking forward to seeing you again when the university reopens. Ci vediamo presto!

I would like to thank the other founding members of the Bob Ross Fan Club, Madeline Zhang and Caulan Rupke, for the trips to Booster Juice, the paint nights, and showing off all the cool things they're up to.

I would like to thank my UTIAS friends who made my time there so enjoyable. Thanks so much to Sohini Chakraborty, Eugene Du, Michael Sleeman, Anthony Webster, Isaac Chung, Geoff Donoghue, Aaron Klein, Kevin Course (see you in my PhD!), Ben Gibson, Adrian Humphry, and George Lu. Special thanks to Keishi Kumashiro for technical feedback, critique, and advice at all stages of this work.

I would like to thank the used Nespresso machine in the corner of the lab. You were always there to keep me going, at least until the pandemic parted us forever.

I would like to thank my parents and grandparents, who spoiled me with the best homecooked and home-grown food. Thanks for letting me live at home well into my twenties.

Finally, I would like to thank Lucy for her companionship over the last year. You are brilliant, hardworking, thoughtful, and endlessly supportive. I'm glad to have you with me.

This work was supported by the US Air Force Office of Scientific Research under Grant FA9550-17-1-0011 (Project Monitor Dr. Chiping Li) and the Ontario Graduate Scholarship. Computations were performed on the Niagara supercomputer at the SciNet HPC Consortium. SciNet is funded by the Canada Foundation for Innovation; the Government of Ontario; Ontario Research Fund - Research Excellence; and the University of Toronto. 


\section{Contents}

1 Introduction $\quad 1$

1.1 Motivation . . . . . . . . . . . . . . . . . 1

1.2 Background . . . . . . . . . . . . . . . . . . 2

1.2.1 Bayes Filter . . . . . . . . . . . . . . . . . . 3

1.2.2 Variational Data Assimilation . . . . . . . . . . . . . . . 3

1.2.3 Ensemble Data Assimilation . . . . . . . . . . . . . . . . . 4

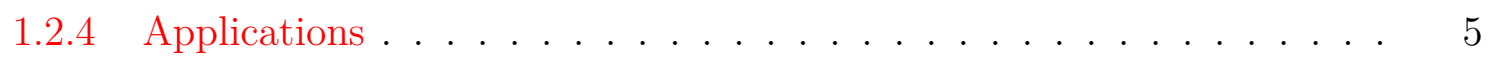

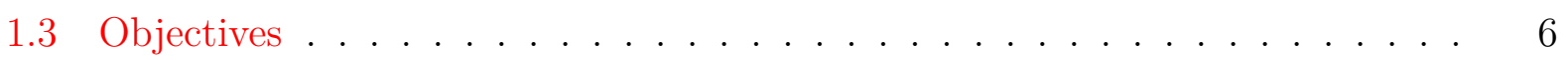

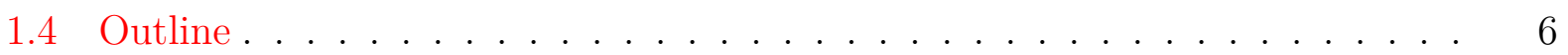

2 Ensemble DA Formulation $\quad 7$

2.1 Problem Definition . . . . . . . . . . . . . . . . . . . . 8

2.2 Ensemble Kalman Filter . . . . . . . . . . . . . . . . . . . . . 9

2.3 Ensemble Transform Kalman Filter . . . . . . . . . . . . . . . . . . 11

2.4 Under-resolved Covariance . . . . . . . . . . . . . . . . . 11

2.5 Resolving Techniques . . . . . . . . . . . . . . . . . . 12

2.5.1 Adaptive Ensemble Size . . . . . . . . . . . . . . . . 13

2.5.2 Inflation . . . . . . . . . . . . . . . . . 14

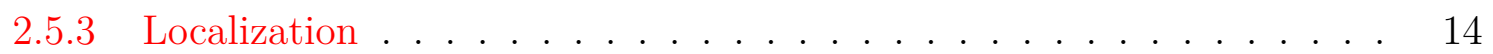

3 Covariance Augmentation $\quad 17$

3.1 Generating Runs . . . . . . . . . . . . . . . . . 18

3.2 Augmented Runs . . . . . . . . . . . . . . . . . . . . . . . 20

3.3 Background Classification . . . . . . . . . . . . . . . . . . . . 21

3.3.1 Distance-Based Classification . . . . . . . . . . . . . 21

3.3.2 Bayesian Classification . . . . . . . . . . . . . . . . 23

3.4 Training Strategy . . . . . . . . . . . . . . . . 26

3.5 Summary . . . . . . . . . . . . . . . . . . 26 
4 Model Test Problems $\quad 28$

4.1 Problem Definitions . . . . . . . . . . . . . . . . . . . . . . 30

4.1 .1 Lorenz 96 Equation . . . . . . . . . . . . . . . . . . . . . . 30

4.1 .2 Lorenz 04 Equation . . . . . . . . . . . . . . . . . . . . . . . 31

4.1 .3 KS Equation . . . . . . . . . . . . . . . . . . . . 34

4.2 Baseline Performance . . . . . . . . . . . . . . . . . . . . . . 35

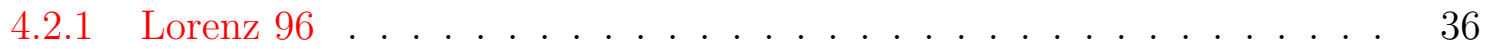

4.2 .2 Lorenz $04 \ldots \ldots \ldots$. . . . . . . . . . . . . . . . . . . . . . . . . 38

4.2 .3 KS Equation . . . . . . . . . . . . . . . . . . . . . 38

4.3 Inflation . . . . . . . . . . . . . . . . . . . 39

4.4 Localization . . . . . . . . . . . . . . . . . . . . . . . . . . . 41

4.5 Augmentation . . . . . . . . . . . . . . . . . . 44

4.5.1 Reproduction Scenario . . . . . . . . . . . . . . . . . . . 44

4.5 .2 Different Trajectory . . . . . . . . . . . . . . . . . . 50

4.5 .3 Parameter Variation $\ldots \ldots \ldots \ldots$

5 Reacting Flow Simulation $\quad 54$

5.1 Problem Definitions . . . . . . . . . . . . . . . . . . . . . . . 54

5.1 .1 Statistically Stationary Flame . . . . . . . . . . . . . . 55

5.1 .2 Extinction Event . . . . . . . . . . . . . . . . 56

5.1 .3 Observation Model . . . . . . . . . . . . . . . . . . . 56

5.2 Assessment Procedure . . . . . . . . . . . . . . . . . . . . . . . . 58

5.3 Baseline Performance . . . . . . . . . . . . . . . . . . . . . . . . 59

5.3 .1 Large Ensemble Size . . . . . . . . . . . . . . . . . . . . 60

5.3 .2 Small Ensemble Size . . . . . . . . . . . . . . . . . . . . . 62

5.4 Augmentation . . . . . . . . . . . . . . . . . . . . . . 65

5.4 .1 Reproduction Scenario . . . . . . . . . . . . . . . . . 65

5.4 .2 Different Trajectory . . . . . . . . . . . . . . . . . . 68

$\begin{array}{llr}6 & \text { Conclusion } & \mathbf{7 0}\end{array}$

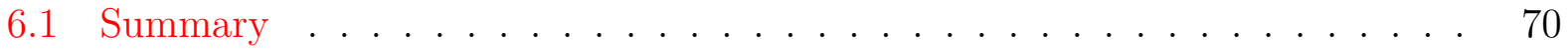

6.2 Limitations and Future Work . . . . . . . . . . . . . . . 71

$\begin{array}{lr}\text { A Ensemble DA Implementation } & \mathbf{7 5}\end{array}$

A.1 Ensemble DA . . . . . . . . . . . . . . . . . . . . 75

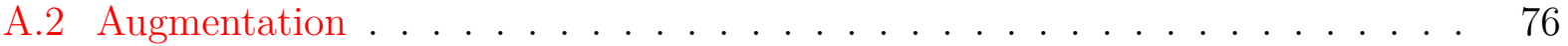


$\begin{array}{ll}\text { B CFD Interfacing } & 77\end{array}$

$\begin{array}{ll}\text { Bibliography } & 78\end{array}$ 


\section{List of Figures}

2.1 Examples of sample covariance matrices from EnKF applied to the KS equation. This colour map is a visualization of the values in the covariance. . . . 12

3.1 A background-generating run . . . . . . . . . . . . . . 18

3.2 An augmented run . . . . . . . . . . . . . . . . . . . . . 20

3.3 Expected value of scale factor $\rho$ that minimizes Wasserstein distance between a $36 \times 36$ identity matrix and its Monte-Carlo approximation, as a function of ensemble size. . . . . . . . . . . . . . . . . . . .

3.4 Probability density function of Wasserstein distance between background and Monte Carlo approximation. . . . . . . . . . . . . . . . . . . . 24

4.1 Reference solution for L96 problem . . . . . . . . . . . . . . . . 31

4.2 Reference solution for L04 problem . . . . . . . . . . . . . . . . . . 33

4.3 Reference solution for KS problem . . . . . . . . . . . . . . . . . 35

4.4 Distributions of error and uncertainty for the ETKF applied to model problems 37

4.5 Distributions of error and uncertainty for the ETKF with inflation applied to model problems . . . . . . . . . . . . . . . . . . . . 4 40

4.6 Distributions of error and uncertainty for the ETKF with localization applied to model problems . . . . . . . . . . . . . . . . . . 42

4.7 Distributions of error and uncertainty for the ETKF with perturbed length scale localization applied to model problems . . . . . . . . . . . . .

4.8 Distributions of error and uncertainty for the ETKF with augmentation applied to model problems in the reproduction scenario . . . . . . . . . . . . . 45

4.9 Distributions of error and uncertainty for the ETKF with augmentation applied to model problems in the reproduction scenario for different $n_{B} \ldots$. . 47

4.10 Distributions of error and uncertainty for the ETKF with augmentation applied to model problems in the reproduction scenario for different $n_{B}$ with Bayesian classification . . . . . . . . . . . . . . . 
4.11 Distributions of error and uncertainty for the ETKF with augmentation applied to model problems in the different trajectory scenario . . . . . . . . .

4.12 Distributions of error and uncertainty for the ETKF with augmentation applied to model problems in the parameter variation scenario . . . . . . . . .

5.1 Statistically stationary diffusion flame. The plot is of non-dimensionalized temperature. The direction of flow is from left to right. The gravity vector points to the left. . . . . . . . . . . . . . . . . .

5.2 Diffusion flame extinction event. The plot is of non-dimensionalized temperature. The direction of flow is from left to right. The gravity vector points to the left. . . . . . . . . . . . . . . . . . . . . 57

5.3 Spatial resolution of observation data in velocity and temperature . . . . . . 58

5.4 Trajectory of nondimensionalized velocity and temperature over one observation period. Measurements taken at centre of domain in a statistically stationary flame case. . . . . . . . . . . . . . . . . . . .

5.5 Example of "checkerboard" effect in statistically stationary flame at time $t=$ 0.5. The plot is of non-dimensionalized vertical velocity. The direction of flow is from left to right. The gravity vector points to the left. . . . . . . . . . . . 60

5.6 Statistically stationary flame - baseline results with large-ensemble ETKF . . 61

5.7 Extinction event - baseline results with large-ensemble ETKF . . . . . . . . 61

5.8 Statistically stationary flame - baseline results with small-ensemble ETKF . 63

5.9 Extinction event - baseline results with small-ensemble ETKF . . . . . . . . 64

5.10 Statistically stationary flame - reproduction scenario augmentation results with small-ensemble ETKF . . . . . . . . . . . . . . 66

5.11 Extinction event - reproduction scenario augmentation results with smallensemble ETKF . . . . . . . . . . . . . . . . . . . .

5.12 Statistically stationary flame - different trajectory augmentation results with small-ensemble ETKF . . . . . . . . . . . . . . . . . . . . . 


\section{Chapter 1}

\section{Introduction}

\subsection{Motivation}

The ability to accurately model turbulent combustion has the potential to greatly reduce the cost and time to develop aircraft jet engines. The development of new gas turbine engines currently may exceed hundreds of millions of dollars [34], as the design process relies on the construction and assessment of prototype designs. This makes design iteration a costly endeavour, providing a strong economic incentive for greater reliance on computational fluid dynamics (CFD). If accurate and tractable computational models are available, fewer design alternatives have to be constructed and tested. Under steady conditions, such as an aircraft in cruise, some engine modelling is feasible with current techniques [11]. In general, however, turbulent reacting flow simulations are not sufficiently accurate or tractable for practical use due to the complexity of coupled chemical and turbulent processes involved [28].

For large-scale turbulent combustion problems, direct numerical simulation is prohibitively expensive due to the extremely wide range of relevant length scales to be captured [27]. Making this problem tractable requires an approach that approximates small-scale phenomena, for which there are two common options. Large-eddy simulation (LES) fully resolves largescale flow behaviour and models the effects of smaller, unresolved scales on resolved flow. Reynolds-averaged Navier-Stokes (RANS) provides a fully time-averaged solution, which is limited in its ability to capture the geometry-dependent physics of large-scale fluid motion and transient phenomena. LES has therefore emerged as the preferred paradigm in reacting flow simulation [28], however Yu et al. [38] and Poinsot [27] collectively identify three persistent sources of difficulty:

- The dynamics are extremely sensitive to uncertainty in initial conditions, boundary conditions, or model parameters, 
- Assumptions that make a model of reacting flow tractable may result in inaccurate parameters or fewer degrees of freedom than required, and

- LES is limited in its ability to model the effect of subfilter scales on resolved scales.

In experimental combustion, high repetition-rate laser diagnostic techniques are able to capture time-correlated data for key state variables. For high Reynolds number flows, however, it is not always possible to measure data on the smallest characteristic length and time scales [33]. A reacting flow is particularly challenging, as not all quantities of interest can be measured and some measurable quantities cannot be interpreted without knowledge of unmeasurable ones [28]. While such time-resolved data have been useful to gain physical understanding, the dynamical information contained in the measurements has, thus far, not been effectively leveraged to directly improve LES models or the ability of LES to mimic behaviour seen in experiments.

Assessment of a gas turbine combustor often involves studying performance trends as a function of operating conditions [34,1]. This is known as a multiple-query scenario, where several distinct evaluations of similar systems are performed. It suggests that a machinelearning approach may be useful to characterizing such a scenario, reducing the computational cost of each evaluation. Such an approach allows for an algorithmic solution which does not rely strongly on user intuition regarding the system under consideration. This thesis contributes to the effective co-utilization of experimental data and LES in multiplequery scenarios by improving the speed and cost of ensemble-based data assimilation without compromising accuracy.

\subsection{Background}

Data assimilation (DA) seeks to incorporate experimental data into a mathematical model of the system of interest (in this case, a reacting flow) to produce a best estimate of the flow state. It may be further used to provide a physics-informed interpolation of sparse experimental data or to infer model parameters. In this thesis however, we are only concerned with estimating the flow state.

In DA, we model the state estimate at a particular time $t$ as a probability distribution. This state probability distribution is propagated forward in time in a two-step process. The first step is the forecast step, which utilizes a mathematical model of the system, known as the process model, to produce a model-based prediction of the state distribution at a future time $t+\Delta t$. The second step is the analysis step, which applies a correction to this state estimate based on experimental observation data. To recognize when a state estimate is discrepant 
with observation data, we require an observation model, which estimates the observation data that would be produced by a given state. Now that we have a state estimate at time $t+\Delta t$ based on observation data up to time $t+\Delta t$, we move forward another increment $\Delta t$ and repeat this two-step process. Note that the process model may be used to propagate a state arbitrarily far forward in time, so the time increment $\Delta t$ corresponds to the period of observation data.

\subsubsection{Bayes Filter}

The ideal solution for this two-step recursive DA process is a Bayes filter. For each time step $k$, the filter estimates the distribution of state $u_{k}$. We start with a distribution $p\left(u_{k-1} \mid v_{1: k-1}^{\text {obs }}\right)$ of the state at time step $k-1$ given observation data $v_{1: k-1}^{\text {obs }}$ from time steps 1 through $k-1$. This comes from an initial condition estimate if $k=1$. In the forecast step, the filter propagates this distribution by

$$
p\left(u_{k} \mid v_{1: k-1}^{\mathrm{obs}}\right)=\int p\left(u_{k} \mid u_{k-1}\right) p\left(u_{k-1} \mid v_{1: k-1}^{\mathrm{obs}}\right) d u_{k-1}
$$

where $p\left(u_{k} \mid u_{k-1}\right)$ is a prediction of the future state $u_{k}$ given the current state $u_{k-1}$, the process model. In the analysis step, we generate a distribution $p\left(u_{k} \mid v_{1: k}^{\text {obs }}\right)$ of the state based on all measurements up to time step $k$. We invoke Bayes rule to obtain

$$
p\left(u_{k} \mid v_{1: k}^{\mathrm{obs}}\right)=\frac{p\left(v_{k}^{\mathrm{obs}} \mid u_{k}\right) p\left(u_{k} \mid v_{1: k-1}^{\mathrm{obs}}\right)}{p\left(v_{k}^{\mathrm{obs}} \mid v_{1: k-1}^{\mathrm{obs}}\right)}=\eta p\left(v_{k}^{\mathrm{obs}} \mid u_{k}\right) p\left(u_{k} \mid v_{1: k-1}^{\mathrm{obs}}\right)
$$

where $p\left(v_{k}^{\text {obs }} \mid u_{k}\right)$ is the likelihood that the observation data is consistent with a given state, the observation model. The coefficient $\eta=1 / p\left(v_{k}^{\text {obs }} \mid v_{1: k-1}^{\text {obs }}\right)$ is a normalization constant.

To use the Bayes filter, we must be able to represent and manipulate arbitrary continuous probability distributions in high-dimensional spaces. This is not possible in general, as we can only implement probability distributions with finite representation, and the integral in (1.1) is intractable. There are two common computationally tractable approximations of the Bayes filter: variational DA and ensemble DA.

\subsubsection{Variational Data Assimilation}

The first approach is variational DA, which includes 3dVar and 4dVar [7, 8, 29]. Variational DA algorithms perform the forecast step in a straightforward manner by propagating a single state estimate, which approximates the mean, using the process model. The analysis 
step involves the optimization of a predefined criterion, i.e., the minimization of a leastsquares cost function, to produce the analysis estimate. This cost function depends on both the forecast error, i.e., the difference between the forecast and analysis estimates, and the observation error, i.e., the difference between the observation data and the observation model applied to the analysis estimate. Each term is scaled appropriately by a respective covariance matrix, representing the uncertainty associated with the source of information. The state distribution therefore is fully represented by the state estimate (i.e., the mean) and covariance.

The uncertainty associated with observation data is knowable a priori to a high degree of certainty, however the uncertainty associated with the forecast state is not [29]. The variational algorithms do not by themselves produce an estimate of this covariance and require another method to supplement them. These forecast covariance estimation methods make the problem tractable with assumptions about characteristics of the covariance. An example is the NMC method [26], which assumes that the statistical structure of the background covariance varies little over 48 hours, thus the forecasts over that period may be used to estimate the background covariance. A more modern example is the analysis-ensemble method [7], which runs an ensemble of independent analysis experiments. In each one, observation data is perturbed with random noise, drawn from a distribution assumed to be representative of observation error. The distribution of forecasts across the ensemble is then used to estimate the background distribution. It is difficult to apply these assumptions to the statistical behaviour of turbulent reacting flow for the reasons mentioned in Section 1.1; the ranges of length and time scales involved may span several orders of magnitude [33]. Ensemble methods, on the other hand, take a purely data-driven approach to estimating the covariance.

\subsubsection{Ensemble Data Assimilation}

The second approach is ensemble DA, which is the focus of this work. This is a newer approach to DA $[14,6,15]$ which leverages increases in computational power to model the state distribution in a Monte-Carlo fashion, i.e., with an ensemble of states that are propagated forward in time together. The forecast covariance is then estimated using the ensemble sample covariance. The prototypical example of this approach is the ensemble Kalman filter (EnKF), which uses the ensemble representation to generalize the classical Kalman filter, allowing it to be applied to large-scale, nonlinear problems. The EnKF is

distinct from a straightforward application of a particle filter [24] because we retain the assumption from the classical Kalman filter that the state distribution is Gaussian, reducing 
the ensemble size necessary to model it. Nevertheless, for complicated dynamical systems, as in atmospheric models, the ensemble size required to produce a statistically-resolved distribution can grow extremely large. This proportionally increases computational cost, as each ensemble member represents another evaluation of the process model. This makes ensemble DA more computationally expensive than variational DA.

There exist two broad categories of methods that aid in resolving the distribution, especially for a small ensemble size: inflation [6,3] and localization [6, 12]. Inflation increases the variance directly by spreading samples about the mean, while localization suppresses spurious long-distance correlations. Inflation requires a factor by which to spread the distribution, which may be fixed or estimated adaptively. Localization requires a characteristic length scale for the system, which it uses to either preserve or suppress apparent correlation terms. There exist common practices to estimate the inflation factor [6], however estimating the length scale requires some prior knowledge of the system. This is feasible for the atmospheric models used in meteorology, where extensive literature is available [21, 36]. Reacting flows however are not as well-characterized. There may also be many characteristic length scales orders of magnitude apart [33], causing localization to either suppress accurate larger-scale correlation terms or permit spurious smaller-scale terms. Detailed formulations of ensemble DA and its resolving techniques are presented in Chapter 2.

\subsubsection{Applications}

DA is commonly used in meteorology, in particular numerical weather prediction (NWP), where sophisticated atmospheric models benefit from correction by sparse weather station data. The variational methods of 3dVar and 4dVar were commonly used historically [29], however the EnKF has seen increasing use over the past decade [15]. With the increase in available computational power, it is becoming more common to see the EnKF used in operational centres for NWP including the Canadian Meteorological Centre and the Italian National Meteorological Center. The National Centers for Environmental Prediction use a "hybrid" arrangement of both ensemble and variational methods.

Though the application of DA to NWP is long-established, its application to reacting flow is still a recent development. In 2019, Labahn et al. [19] successfully applied the EnKF to a turbulent jet exhausting into a stationary fluid. Another study by Labahn et al. [18] successfully applied the EnKF to a non-premixed turbulent flame, serving as a proof of concept for turbulent combustion as a whole. Yu et al. [38] successfully applied the EnKF to a reduced-order model of a premixed flame to not only correct the state estimate, but to also correct model parameters, making the reduced-order model more accurate even without 
observation data. Yu et al. [39] used similar techniques in the development of an original reduced-order model for a ducted premixed flame. As discussed in Section 1.1, reacting flow problems in practice are often multiple-query problems, however to our knowledge, no published research has specifically considered strategies for improving DA performance in multiple-query scenarios.

\section{$1.3 \quad$ Objectives}

The objective of this thesis is to reduce the cost of ensemble-based DA with an emphasis on multiple-query scenarios while controlling the state estimation error. We achieve this with a data-driven method for estimating the covariance which we term augmentation, where information from statistically-resolved runs is retained and drawn upon in subsequent under-resolved runs. This statistical information characterizes the system and reduces the computational cost while maintaining a desired level of accuracy.

In addition, we assess our augmentation method using three model problems and two cases of reacting flow. We compare its performance to the unaugmented EnKF and the other aforementioned resolving techniques of inflation and localization. We consider cases where the statistical information does not fully match the behaviour of the system over the time interval under consideration, testing the generalizability of the augmentation technique. We focus in particular on the ensemble size reduction achievable for a desired level of estimation error.

\subsection{Outline}

There are six chapters in this thesis. We present a detailed formulation of ensemble DA and its associated resolving techniques in Chapter 2, which extends the discussion presented in Section 1.2.3. In Chapter 3, we present our method of augmentation. We apply the ensemble DA techniques and augmentation to model problems in Chapter 4 and reacting flow problems in Chapter 5. These are synthetic problems, meaning that the "ground truth" reference solution and the observation data are both produced artificially. We show that augmentation allows a reduction in ensemble size, and hence cost, without reducing accuracy. We conclude with discussion of limitations and future work in Chapter 6. 


\section{Chapter 2}

\section{Ensemble DA Formulation}

The Bayes filter described in Section 1.2.1 is the canonical solution to the problem of recursive DA, however it requires the ability to model arbitrary distributions and integrate them, which is not possible in general with a finite representation. We make this problem tractable with the ensemble DA approach introduced in Section 1.2.3. We assume that all probability distributions are Gaussian (i.e., can be fully represented with a mean and covariance) and that the state and observation are finite vectors $\mathbf{u}$ and $\mathbf{v}^{\text {obs }}$ respectively.

As mentioned in Section 1.4, we consider synthetic test cases in this thesis. We begin a synthetic test case by first using the process model to propagate an initial state $\mathbf{u}_{0}$ forward in time, producing the "ground truth" solution $\mathbf{u}_{k}$, where the subscript $k \in\left[1, n_{t}\right]$ denotes the observation time index. We then produce the corresponding synthetic observation data $\mathbf{v}_{k}^{\text {obs }}, k \in\left[1, n_{t}\right]$, by applying the observation model to the ground truth. With this synthetic dataset, we finally perform DA on an ensemble of solutions using $\mathbf{v}_{k}^{\text {obs }}$ as the measurements of the system state. In the forecast and analysis steps, we use the same process model and observation model used to produce the ground truth and observation data, so there is no inherent model deficiency.

We start this section by presenting a detailed problem definition, including the assumed forms of the process model and observation model. We then present two applicable methods of ensemble DA: the ensemble Kalman filter (EnKF), and a modified form of it known as the ensemble transform Kalman Filter (ETKF). The ETKF is applied to test problems in Chapters 4 and 5. We then discuss the filter performance issues caused by small ensemble sizes and the existing supplemental techniques to address them. 


\subsection{Problem Definition}

The first step of our synthetic test problem is to generate a "ground truth" reference solution $\left\{\mathbf{u}_{1}, \ldots, \mathbf{u}_{n_{t}}\right\}$, which we use to both produce synthetic observation data and to determine the error in the state estimate. We start with an initial state vector $\mathbf{u}_{0}$, which is specified for each particular test case in Chapters 4 and 5. We then propagate the initial state forward using the process model, which takes the form

$$
\mathbf{u}_{k}=G\left(\mathbf{u}_{k-1}\right)
$$

for a nonlinear operator $G(\cdot)$. The operator $G(\cdot)$ represents many successive integration time-steps of a differential equation or many successive time-steps of the fluid conservation equations (i.e. between observation times), depending on the test case of interest. We apply the process model for each time step $k \in\left[1, n_{t}\right]$, producing the ground truth solution. Note that the process model corresponds to the term $p\left(u_{k} \mid u_{k-1}\right)$ in the forecast step (Eq. (1.1)), which estimates how the state $\mathbf{u}_{k-1}$ propagates forward in time to $\mathbf{u}_{k}$. The process model may include a noise term to represent model inadequacy, but because this is a synthetic test problem, there is no model inadequacy present. To apply this deterministic process model to state probability distributions, we consider an ensemble representation as detailed in Section 2.2.

We then generate the synthetic observation data $\left\{\mathbf{v}_{1}^{\text {obs }}, \ldots, \mathbf{v}_{n_{t}}^{\text {obs }}\right\}$ which corresponds to the ground truth solution. We do this by applying the observation model to the ground truth, which takes the form

$$
\mathbf{v}_{k}^{\mathrm{obs}}=H\left(\mathbf{u}_{k}\right)+\mathbf{r}_{k},
$$

for a nonlinear operator $H(\cdot)$ and a Gaussian noise term $\mathbf{r}_{k} \sim \mathcal{N}(\mathbf{0}, \mathbf{R})$. The operator $H(\cdot)$ models, for instance, points in space where sensor data is available or the acquisition of PIV data associated with the ground-truth LES state, again depending on the test case of interest. The noise term models noise in the observation data due to imperfect measurements. Note that the observation model corresponds to the term $p\left(v_{k} \mid u_{k}\right)$ in the analysis step (Eq. (1.2)), which estimates how the observation $\mathbf{v}_{k}$ is produced by the state $\mathbf{u}_{k}$. We apply the observation model for each time step $k \in\left[1, n_{t}\right]$, producing the observation data.

Ensemble DA techniques represent the state estimate and associated uncertainty as an empirical probability distribution with an ensemble of states $\left\{\mathbf{u}_{j}\right\}_{j=1}^{n_{\text {en }}}$. The Gaussian probability distribution is represented fully with the mean and covariance, which ensemble DA estimates with the sample mean and sample covariance of the ensemble. In general, the ensemble is initialized based on prior knowledge of the state distribution. For the synthetic 
test cases we consider in Chapters 4 and 5, the initial ensemble members are drawn randomly from the history of the ground truth

$$
\left\{\mathbf{u}_{k_{1}}, \mathbf{u}_{k_{2}}, \ldots, \mathbf{u}_{k_{n_{\mathrm{en}}}}\right\}, \quad k_{1}, \ldots, k_{n_{\mathrm{en}}} \sim \mathcal{U}\left\{1, \ldots, n_{t}\right\}
$$

where $\mathcal{U}\{\cdot\}$ is the discrete uniform distribution. This ensures that the ensemble members are both physically plausible and poorly converged. The task of the filter therefore is to converge the ensemble around the ground truth and subsequently "track" the ground truth, preventing error growth.

\subsection{Ensemble Kalman Filter}

The EnKF propagates the ensemble state estimate forward in time in a two-step process, following the Bayes filter detailed in Chapter 1. The first step is the forecast step, where we apply the process model from (2.1) to each ensemble member

$$
\mathbf{u}_{j k \mid k-1}=G\left(\mathbf{u}_{j_{k-1 \mid k-1}}\right), \quad j=1, \ldots, n_{\mathrm{en}} .
$$

to produce the forecast estimate of the state. The notation $\mathbf{u}_{j_{k-1 \mid k-1}}$ indicates that this ensemble member $j$ is from the state distribution at time step $k-1$ given observation data up to time step $k-1$. When we perform the forecast step, the forecast estimate ensemble member $\mathbf{u}_{j k \mid k-1}$ is from the state distribution at time step $k$ given observation data up to time step $k-1$. We estimate the forecast covariance $\mathbf{C}_{k \mid k-1}$ using the sample covariance of the ensemble,

$$
\mathbf{C}_{k \mid k-1}=\frac{1}{n_{\mathrm{en}}-1} \sum_{j=1}^{n_{\mathrm{en}}}\left(\mathbf{u}_{j k \mid k-1}-\overline{\mathbf{u}}_{k \mid k-1}\right)\left(\mathbf{u}_{j k \mid k-1}-\overline{\mathbf{u}}_{k \mid k-1}\right)^{T}
$$

where the overline denotes the ensemble mean. To rewrite this equation without the summation, we define an ensemble matrix where each column is an ensemble member

$$
\mathbf{U}_{k \mid k-1}=\left[\mathbf{u}_{1 k \mid k-1}, \ldots, \mathbf{u}_{n_{\mathrm{en} k \mid k-1}}\right]
$$

and we define the corresponding ensemble mean and deviation matrices

$$
\begin{gathered}
\overline{\mathbf{U}}_{k \mid k-1}=\left[\overline{\mathbf{u}}_{k \mid k-1}, \ldots, \overline{\mathbf{u}}_{k \mid k-1}\right] \\
\tilde{\mathbf{U}}_{k \mid k-1}=\left[\mathbf{u}_{1 k \mid k-1}-\overline{\mathbf{u}}_{k \mid k-1}, \ldots, \mathbf{u}_{n_{\mathrm{en} k \mid k-1}}-\overline{\mathbf{u}}_{k \mid k-1}\right] .
\end{gathered}
$$


The covariance can then be expressed as

$$
\begin{aligned}
\mathbf{C}_{k \mid k-1} & =\frac{1}{n_{\mathrm{en}}-1}\left(\mathbf{U}_{k \mid k-1}-\overline{\mathbf{U}}_{k \mid k-1}\right)\left(\mathbf{U}_{k \mid k-1}-\overline{\mathbf{U}}_{k \mid k-1}\right)^{T} \\
& =\frac{1}{n_{\mathrm{en}}-1} \tilde{\mathbf{U}}_{k \mid k-1} \tilde{\mathbf{U}}_{k \mid k-1}^{T},
\end{aligned}
$$

in terms of the ensemble matrices. We also define the observation ensemble

$$
\mathbf{V}_{k \mid k-1}=\left[\begin{array}{lll}
\mathbf{v}_{1 k \mid k-1} & \ldots & \mathbf{v}_{n_{\mathrm{en} k \mid k-1}}
\end{array}\right]=\left[\begin{array}{lll}
H\left(\mathbf{u}_{1 k \mid k-1}\right) & \ldots & H\left(\mathbf{u}_{n_{\mathrm{en} k \mid k-1}}\right)
\end{array}\right]
$$

calculated by applying the observation model from (2.2) (neglecting the noise term) to each member the forecast ensemble. The corresponding mean and deviation matrices $\overline{\mathbf{V}}_{k \mid k-1}$ and $\tilde{\mathbf{V}}_{k \mid k-1}$ are analogous to (2.7). We then find the observation sample covariance

$$
\begin{aligned}
\mathbf{D}_{k \mid k-1} & =\frac{1}{n_{\mathrm{en}}-1}\left(\mathbf{V}_{k \mid k-1}-\overline{\mathbf{V}}_{k \mid k-1}\right)\left(\mathbf{V}_{k \mid k-1}-\overline{\mathbf{V}}_{k \mid k-1}\right)^{T} \\
& =\frac{1}{n_{\mathrm{en}}-1} \tilde{\mathbf{V}}_{k \mid k-1} \tilde{\mathbf{V}}_{k \mid k-1}^{T}
\end{aligned}
$$

in a manner analogous to (2.8).

Now that we have a forecast estimate, we have propagated the state from time $k-1$ to $k$. For the analysis step, we incorporate the synthetic observation data $\mathbf{v}_{k}^{\text {obs }}$ to refine our forecast estimate. We calculate the analysis estimate by

$$
\mathbf{u}_{j k \mid k}=\mathbf{u}_{j k \mid k-1}+\mathbf{K}_{k}\left(\mathbf{v}_{k}^{\mathrm{obs}}-\mathbf{v}_{j_{k \mid k-1}}\right), \quad j=1, \ldots, n_{\mathrm{en}},
$$

which applies a linear update to each ensemble member. The gain matrix $\mathbf{K}_{k}$ is the Kalman gain, which is given by

$$
\mathbf{K}_{k}=\frac{1}{n_{\mathrm{en}}-1} \tilde{\mathbf{U}}_{k \mid k-1} \tilde{\mathbf{V}}_{k \mid k-1}^{T}\left(\mathbf{D}_{k \mid k-1}+\mathbf{R}\right)^{-1}
$$

and is chosen to minimize the mean-square error in the state estimate. The matrix $\mathbf{R}$ is the covariance of the noise term in the observation model (2.2). For a linear observation model $H(\cdot)=\mathbf{H}$, the Kalman gain is given by

$$
\mathbf{K}_{k}=\mathbf{C}_{k \mid k-1} \mathbf{H}^{T}\left(\mathbf{H C}_{k \mid k-1} \mathbf{H}^{T}+\mathbf{R}\right)^{-1}
$$

which makes explicit the dependence on forecast covariance $\mathbf{C}_{k \mid k-1}$. Each test problem considered in this work has a corresponding observation model which is linear, therefore (2.13) 
is the relevant calculation. We refer to Appendix A.1 for a memory-efficient formulation for implementation.

\subsection{Ensemble Transform Kalman Filter}

When we use the Kalman gain in the analysis step and the distribution is well-resolved (i.e., the ensemble size is large), then the covariance analysis step is equivalent to

$$
\mathbf{C}_{k \mid k}=\left(\mathbf{I}-\mathbf{K}_{k} \mathbf{H}\right) \mathbf{C}_{k \mid k-1},
$$

which is known as the Kalman identity. For more practical ensemble sizes however, this is not the case. To preserve the Kalman identity for small ensemble sizes, the ensemble transform Kalman filter (ETKF) performs an alternate analysis step. It separately propagates the ensemble mean and member deviations through the analysis step. The forecast step and Kalman gain remain as they are in the EnKF.

The analysis step (2.11) is applied only to the ensemble mean,

$$
\overline{\mathbf{u}}_{k \mid k}=\overline{\mathbf{u}}_{k \mid k-1}+\mathbf{K}_{k}\left(\mathbf{v}_{k}^{\text {obs }}-\overline{\mathbf{v}}_{k \mid k-1}\right),
$$

and the Kalman identity is then enforced by a separate transformation of the normalized ensemble deviation matrix $\hat{\mathbf{U}}_{k \mid k-1}=\frac{1}{\sqrt{n_{\mathrm{en}}-1}} \tilde{\mathbf{U}}_{k \mid k-1}$. For this we define a transformation matrix

$$
\begin{gathered}
\mathbf{T}_{k}=\left(\mathbf{I}_{n_{\mathrm{en}}}+\hat{\mathbf{U}}_{k \mid k-1}^{T} \mathbf{H}_{k}^{T} \mathbf{R}^{-1} \mathbf{H}_{k} \hat{\mathbf{U}}_{k \mid k-1}\right)^{-1}, \\
\tilde{\mathbf{U}}_{k \mid k}=\tilde{\mathbf{U}}_{k \mid k-1} \mathbf{T}_{k}^{1 / 2},
\end{gathered}
$$

where $\mathbf{I}_{n_{\mathrm{en}}}$ is the $n_{\mathrm{en}} \times n_{\mathrm{en}}$ identity matrix. Finally, we add the deviations back to the analysis mean to reobtain the analysis ensemble matrix.

\subsection{Under-resolved Covariance}

Because the forecast covariance $\mathbf{C}_{k \mid k-1}$ is used to calculate the Kalman gain $\mathbf{K}_{k}$, accurately resolving the covariance is critical to the filter's performance. This is determined by the ensemble size, which is in turn determined by the quantity of independent modes in the system. An underlying "true" covariance with many significant eigenmodes will require a larger ensemble size to properly represent, as this requires a covariance matrix of higher rank. 


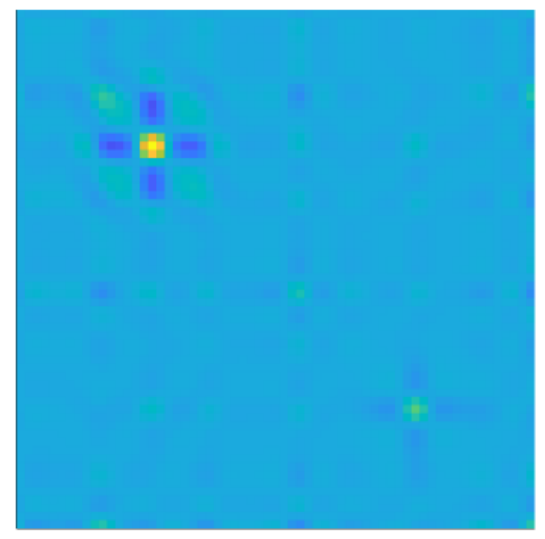

(a) Ensemble size 6, rank-deficient

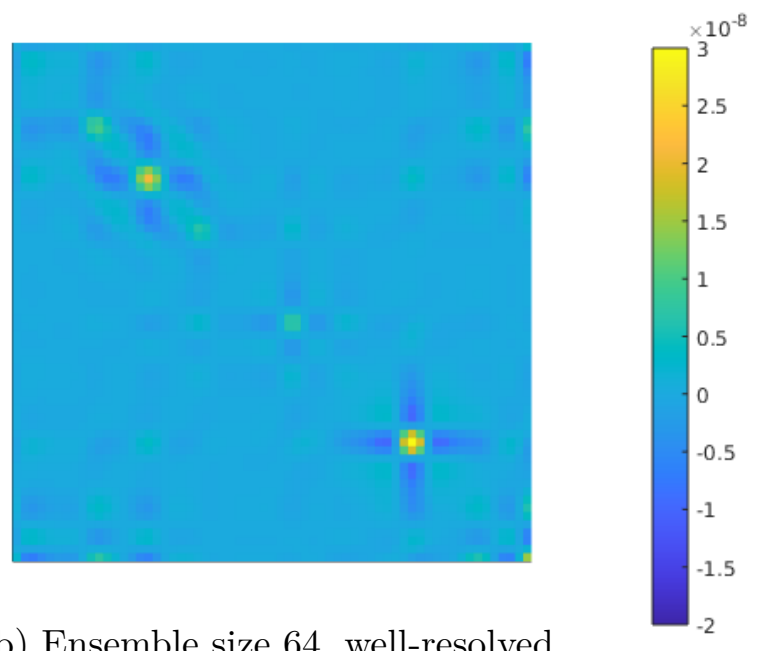

(b) Ensemble size 64, well-resolved

Figure 2.1: Examples of sample covariance matrices from EnKF applied to the KS equation. This colour map is a visualization of the values in the covariance.

An example is presented in Figure 2.1 of a well-resolved and an under-resolved sample covariance. These are obtained from applying the ETKF to the KS Equation, one of the model problems considered in Chapter 4. The rank-limited approximation in Figure 2.1a contains spurious cross-correlation terms and underestimates the magnitude of most variance terms along the diagonal. In practice this leads to an over-converged covariance, i.e., the spread of the ensemble is much smaller than the error in the state estimate. The state then becomes resistant to correction in the analysis step of the filter.

One should note from the analysis step (2.11) and the definition of the Kalman gain (2.12) that the linear update ultimately lies in the column space of the ensemble deviation matrix $\tilde{\mathbf{U}}_{k \mid k-1}$. The ensemble members form a basis for the linear correction applied by the analysis step. An undersized ensemble leads to a heavily restricted vector space, increasing the likelihood that the desired update is outside the column space of $\tilde{\mathbf{U}}_{k \mid k-1}$ and thus cannot be applied. As we will demonstrate, this has a greater effect on the performance of an undersized ensemble than underestimated covariance.

\subsection{Resolving Techniques}

To avoid the consequences of an under-resolved sample covariance, there exist three broad categories of resolving techniques. The first is adaptive ensemble sizing, which estimates when the eigenmodes present in the covariance sufficiently capture the system dynamics. If it determines the ensemble is not appropriately sized, it adds or removes members as necessary. The second is inflation, which seeks to prevent false convergence of the ensemble 
members by spreading the ensemble members from the mean, increasing the covariance. The third is localization, which seeks to suppress spurious correlation terms in the sample covariance by imposing a limiting correlation length appropriate to the system. Inflation and localization may be considered artificial resolving techniques, as they attempt to resolve the sample covariance without additional ensemble members.

\subsubsection{Adaptive Ensemble Size}

To ensure that the ensemble is sufficiently but not excessively sized, an adaptive method may be used. The method considered in this work is one by Uzunoglu et al. [35], which uses the decay of Shannon entropy with eigenmodes in the ensemble to determine whether the ensemble is undersized, oversized, or appropriately sized.

The method starts with a proper orthogonal decomposition (POD) of the forecast covariance $\mathbf{C}_{k \mid k-1}$, i.e., finding the eigenvalues $\lambda_{j}$ for $j=1, \ldots, n_{\mathrm{en}}-1$. Note that the rank of the covariance matrix is $n_{\mathrm{en}}-1$. The total variance can be found by taking the sum of the eigenvalues,

$$
s^{2}=\sum_{j=1}^{n_{\mathrm{en}}-1} \lambda_{j},
$$

which we then use to find the variance percentage for each eigenvector,

$$
s_{j}^{2}=\frac{\lambda_{j}}{s^{2}} .
$$

We then find the Shannon entropy at a particular ensemble size $n_{\text {en }}$ in terms of the variance percentage,

$$
H_{s}\left(n_{\mathrm{en}}\right)=-\sum_{j=1}^{n_{\mathrm{en}}-1} s_{j}^{2} \ln \left(s_{j}^{2}\right),
$$

which serves as a measure of information content in the ensemble. We would like to retain as much information as possible in our ensemble, which is equivalent to maximizing (2.19). We therefore seek the ensemble size $n_{\mathrm{en}}$ where $H_{s}\left(n_{\mathrm{en}}+1\right)-H_{s}\left(n_{\mathrm{en}}\right) \approx 0$. Beyond this ensemble size, additional ensemble members contribute little to resolving the covariance.

We apply this adaptive method by starting the filter with a generous ensemble size and setting a minimum threshold for $H_{s}\left(n_{\mathrm{en}}+1\right)-H_{s}\left(n_{\mathrm{en}}\right)$. If $H_{s}\left(n_{\mathrm{en}}+1\right)-H_{s}\left(n_{\mathrm{en}}\right)$ goes below this threshold, the ensemble size is reduced. The method can also be used to increase ensemble size, though this requires an expanded basis from which to sample new members. Uzunoglu et al. suggests sampling from a "long-exposure" covariance which includes ensemble members from previous time steps, though this function is not necessary for our purposes. It may be noted however that the method of covariance augmentation we propose in Chapter 3 can be 
similarly used to produce these extra members.

\subsubsection{Inflation}

The first artificial resolving technique we consider is covariance inflation. Inflation is done to prevent an undersized ensemble from converging toward the mean which, as discussed in Section 2.4, would make the state estimate resistant to correction in the analysis step. It does this by artificially spreading the ensemble members from the mean by an inflation factor $[3,6,20]$. This is performed for each ensemble member according to

$$
\mathbf{u}_{j k \mid k-1} \leftarrow \rho\left(\mathbf{u}_{j k \mid k-1}-\overline{\mathbf{u}}_{k \mid k-1}\right)+\overline{\mathbf{u}}_{k \mid k-1}, \quad j=1, \ldots, n_{\mathrm{en}}
$$

for an inflation factor $\rho>1$. This is equivalent to

$$
\mathbf{U}_{k \mid k-1} \leftarrow \rho \tilde{\mathbf{U}}_{k \mid k-1}+\overline{\mathbf{U}}_{k \mid k-1}
$$

or

$$
\mathbf{C}_{k \mid k-1} \leftarrow \rho^{2} \mathbf{C}_{k \mid k-1}
$$

\section{Choosing the Inflation Factor}

The inflation factor may either be a fixed value or calculated by an adaptive method. According to Evensen [6], a common fixed value is $\rho=1.05$.

The method considered here is an adaptive method from Anderson [3]. We first define a $n_{u} \times n_{\mathrm{en}}$ matrix $\mathbf{N}$ whose rows contain normally distributed random numbers with sample mean 0 and sample standard deviation 1 . We then pass $\mathbf{N}$ through the analysis step of our filter in place of $\mathbf{U}_{k \mid k-1}$ to obtain $\mathbf{N}_{k}$. Finally we find the mean standard deviation $s_{N}$ of the rows of $\mathbf{N}_{k}$ and define the inflation factor $\rho=1 / s_{N}$.

Though inflation does increase the ensemble spread, it is a rank-preserving operation. If the performance is limited primarily by the rank-deficient covariance, then inflation is not a useful technique. It also may be the case, as we will see in Chapter 4, that undersized ensembles do not necessarily underestimate the covariance, making inflation irrelevant.

\subsubsection{Localization}

Localization is a technique that aims to suppress spurious long-distance correlation terms in the forecast covariance. There are two primary localization techniques we will consider here: 
covariance localization which masks the state covariance $\mathbf{C}_{k \mid k-1}$, and observation localization which masks the observation covariance $\mathbf{R}$ [12].

\section{Covariance Localization}

Localization of the state covariance is done by first creating a $n_{u} \times n_{u}$ mask matrix $\mathbf{M}$ with elements $0<m_{a b}<1$, and then producing a localized sample covariance by

$$
\mathbf{C}_{k \mid k-1} \leftarrow \mathbf{M} \circ \mathbf{C}_{k \mid k-1},
$$

where $\circ$ is the Schur (elementwise) product. A common choice of mask is the Gaussian function

$$
m_{a b}=\exp \left(\frac{-d(a, b)^{2}}{2 L^{2}}\right),
$$

where $d(a, b)$ is the distance between nodes $a \in\left[1, n_{u}\right]$ and $b \in\left[1, n_{u}\right]$ and $L$ is our chosen correlation length scale [12]. Because covariance localization is a rank-increasing operation, it requires forming the full covariance matrix, which is not feasible for large problems [12]. Covariance localization is acknowledged here for completeness, but is not applied to our test problems.

\section{Observation Localization}

Localization of the observation covariance is done by similarly first creating a $n_{v} \times n_{v}$ mask matrix $\mathbf{M}$, however this one has diagonal elements $m_{b b} \geq 1$. The mask function above may be modified to obtain

$$
m_{i, b b}=\exp \left(\frac{+d(i, b)^{2}}{2 L^{2}}\right),
$$

where the distance is between grid point $i \in\left[1, n_{u}\right]$ and observation node $b \in\left[1, n_{v}\right]$. Because the mask matrix is specific to the grid point under consideration, we denote the observation mask matrix as $\mathbf{M}_{i}$. We then produce a localized observation covariance

$$
\mathbf{R}_{i} \leftarrow \mathbf{M}_{i} \circ \mathbf{R},
$$

which consequently is also specific to the grid point $i$. When this localized observation covariance is used in (2.12), it produces a grid-point-specific Kalman gain $\mathbf{K}_{i, k}$. We form the final localized Kalman gain $\mathbf{K}_{k}$ row-by-row: the $i$ th row of $\mathbf{K}_{k}$ is the $i$ th row of $\mathbf{K}_{i, k}$. By increasing the observation covariance at grid points which are far from observation nodes, this localization technique reduces the effect of the analysis update far from observation nodes. 
Because $\mathbf{R}$ can be fully represented as a sparse diagonal matrix, observation localization is the localization technique we apply to our test problems.

\section{Choosing the Correlation Length Scale}

In practice (as will be demonstrated in Chapter 4), the performance of localization is extremely dependent on the choice of length scale. We therefore require an accurate understanding of the dynamical system to avoid compromising performance with a poorly-chosen length scale. As DA is most commonly applied in meteorology, length scale estimation for localization has been extensively studied in that context [36, 21]. For novel problems, however, prior understanding of the system is more limited, making it more difficult to estimate a reasonable length scale and apply localization.

Note also that because we must assume and enforce a length scale, it is possible for well-resolved correlations to be suppressed. This can occur in any dynamical system that exhibits some periodic behaviour, as is often true for reacting flows. There may still be a net improvement to accuracy when sample covariance is severely underresolved, however if ensemble size is sufficiently large, localization can only reduce the accuracy of our sample covariance. For localization to improve the accuracy of our ensemble filter, the dynamical system must be underresolved and should be dominated by short-distance correlation terms.

The assumption of one dominant length scale is also unlikely to be true in the context of reacting flows, where different structures within the flow may have associated length scales that span several orders of magnitude [33]. Any choice of length scale for localization will either result in suppressing genuine larger-scale correlation terms or permitting spurious smaller-scale terms.

For systems with multiscale dynamics, localization may still provide a net benefit. In such a case, the correlation length scale may be chosen empirically to best compromise between falsely-suppressed long-distance correlations and correctly-suppressed spurious correlations [31]. Numerical study suggests the length scale is not strongly affected by changes to model resolution, but depends mostly on the dynamics and observation density [37], although a comprehensive localization theory for multiscale systems remains an area of active research. 


\section{Chapter 3}

\section{Covariance Augmentation}

The novel method proposed in this work is covariance augmentation, which takes a datadriven approach to artificially resolving the forecast covariance in a two-phase process.

1. A generating run performs the DA with a sufficiently large ensemble size to resolve the sample covariance. Information from this generating run is retained in a set of distributions, each of which is represented by an ensemble.

2. Subsequent augmented runs perform the DA with an undersized ensemble, perhaps extremely so, which we then augment by supplementing the deficient natural ensemble with artificial ensemble members. These artificial members are drawn from an appropriate distribution selected from the set produced in the generating run.

These artificial members are so named because they are not produced by evaluating the process model with every forecast step, unlike the "natural" ensemble members. They are only used to artificially resolve the forecast covariance and improve the quality of the analysis update.

This method is designed for multiple-query scenarios, where similar simulations are performed across a wide parameter space. Under normal circumstances, the computational cost scales linearly with $n_{f}$, the number of scenarios considered. If we assume that the simulation model is much more costly than the DA, then the runtime complexity is $O\left(n_{\mathrm{en}} n_{f}\right)$ for $n_{f}$ EnKF runs of a fixed $n_{\text {en }}$.

When we apply our filter to many similar scenarios, each generating run further "trains" our statistical model of the system. The more resolved this statistical model, the more that augmented runs may rely on the artificial members generated and reduce the natural ensemble size $n_{\text {en }}$. For a given level of accuracy, the complexity of a multiple-query problem is now $O\left(n_{\mathrm{en}}{ }^{\prime} n_{f}\right)$, where $n_{\mathrm{en}}{ }^{\prime}<n_{\mathrm{en}}$ is the size of the "natural" ensemble. 


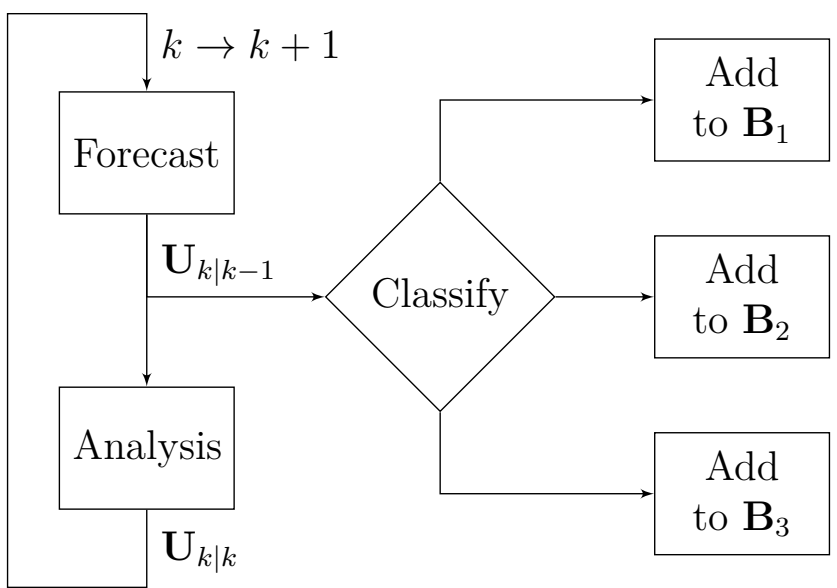

Figure 3.1: A background-generating run

Augmentation is a general framework for training an ensemble filter with model-specific data, allowing it to reduce its ensemble size, and thus computational cost, while retaining accurate performance. Ours is only one potential realization of this framework. A library of distributions that is dense and comprehensive of model behaviour may almost perfectly mimic a well-resolved ensemble, leading to very little difference between well-informed augmentation and a large ensemble. As we see in Chapters 4 and 5, this is achievable in best-case scenarios, where the generating run and augmented run are chosen to match as closely as possible. In more practical scenarios, augmentation's performance is determined by

1. The classification scheme: how a natural forecast ensemble is matched to the appropriate distribution from the library, and

2. The training strategy: the choice of generating runs which produces the library of distributions.

We start this chapter with a discussion of the structure and mechanics of generating runs and augmented runs. We then present two methods of background classification and briefly discuss training strategy. We then finish with a chapter summary.

\subsection{Generating Runs}

The generating run is an unaugmented run of DA that produces a set of $n_{B}$ background covariance matrices $B=\left\{\mathbf{B}_{1}, \mathbf{B}_{2}, \ldots, \mathbf{B}_{n_{B}}\right\}$. This background set will be used to augment the natural ensemble to artificially resolve the forecast covariance in future augmented runs. The ensemble size in a generating run must therefore be large enough to ensure that the sample covariance is statistically converged. 
To introduce the idea of background set in the simplest setting, consider the case of a background set with a single covariance matrix $\mathbf{B}$. The forecast deviation ensemble $\tilde{\mathbf{U}}_{k \mid k-1}$ from each time step is retained, forming the background ensemble

$$
\tilde{\mathbf{W}}=\left[\begin{array}{lllll}
\tilde{\mathbf{U}}_{1 \mid 0} & \ldots & \tilde{\mathbf{U}}_{k \mid k-1} & \ldots & \tilde{\mathbf{U}}_{n_{t} \mid n_{t}-1}
\end{array}\right]
$$

which is then used to estimate the background covariance

$$
\mathbf{B}=\frac{1}{n_{\mathrm{en} B}-1} \tilde{\mathbf{W}} \tilde{\mathbf{W}}^{T}
$$

for $n_{\mathrm{en} B}=n_{t} n_{\mathrm{en}}$ background ensemble members. This produces a "long-exposure" background distribution which retains and emphasizes steady behaviour.

In many physical systems however, the statistical behaviour can be highly unsteady and therefore contextual. So rather than only one background, our augmentation method generates and draws from the set of $n_{B}$ backgrounds. The structure of a generating run with $n_{B}=3$ backgrounds is illustrated in Figure 3.1. For each time step, the natural ensemble is classified into one of the backgrounds in the set. It is then appended to the corresponding background ensemble

$$
\tilde{\mathbf{W}}_{\ell} \leftarrow\left[\tilde{\mathbf{W}}_{\ell} \tilde{\mathbf{U}}_{k \mid k-1}\right],
$$

which leads to an updated background covariance $\mathbf{B}_{\ell}=\frac{1}{n_{\mathrm{en} B}-1} \tilde{\mathbf{W}}_{\ell} \tilde{\mathbf{W}}_{\ell}^{T}$. As the number of forecast ensembles in each background increases, the backgrounds converge on a comprehensive set of $n_{B}$ statistical regimes for the system. The classification scheme we use is detailed in Section 3.3.

When the generating run concludes, we refine the background clustering by applying the naive $k$-means clustering algorithm to the background set. For each forecast ensemble $\tilde{\mathbf{U}}_{k \mid k-1}$ in each background ensemble $\tilde{\mathbf{W}}_{\ell}$,

1. Reclassify $\tilde{\mathbf{U}}_{k \mid k-1}$, finding a matching background ensemble $\tilde{\mathbf{W}}_{\ell^{\star}}$, where $\ell^{\star} \in\left[1, n_{B}\right]$ is the index of the matching background.

2. If $\ell^{\star}=\ell$, leave $\tilde{\mathbf{U}}_{k \mid k-1}$ in $\tilde{\mathbf{W}}_{\ell}$.

3. If $\ell^{\star} \neq \ell$, remove $\tilde{\mathbf{U}}_{k \mid k-1}$ from $\tilde{\mathbf{W}}_{\ell}$ and append it to $\tilde{\mathbf{W}}_{\ell^{\star}}$.

Repeat the above until no more ensembles are reclassified into different backgrounds. Convergence is not guaranteed in general; however, it is achieved regularly in practice. With $k$-means concluded, the ensembles are clustered into Voronoi cells. This minimizes variance between forecast ensembles within each background, providing a statistical regime classification given the forecast ensembles from the generating run. 


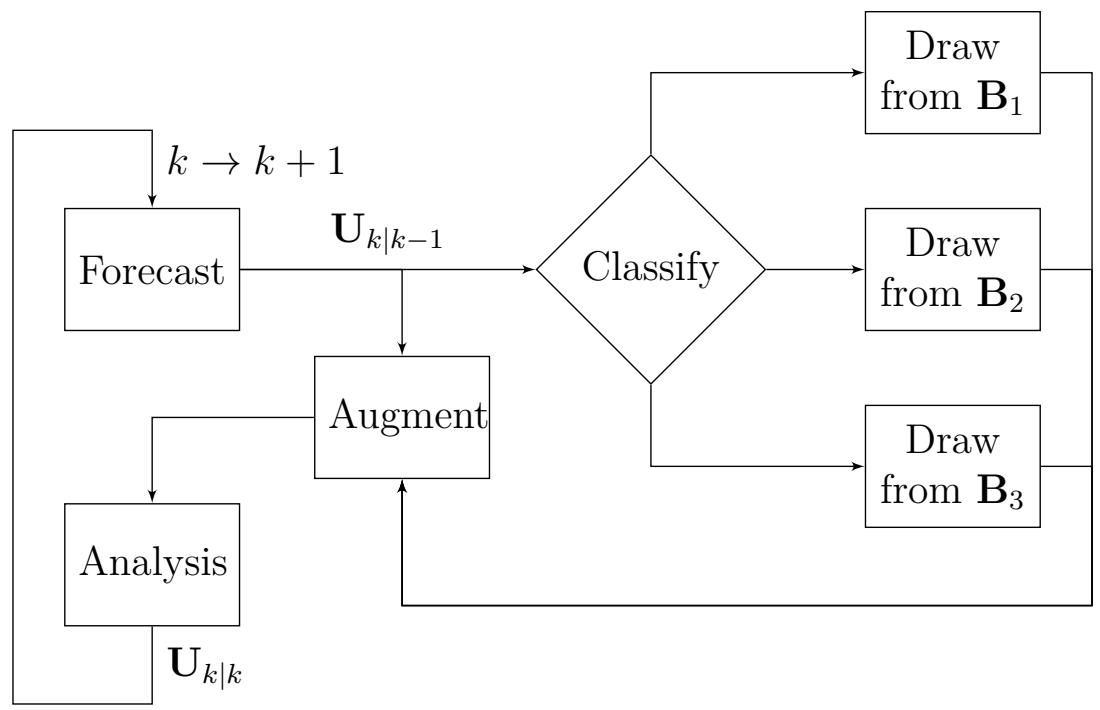

Figure 3.2: An augmented run

\subsection{Augmented Runs}

The augmented run is a run of DA where the forecast ensemble is augmented with artificial members $\mathbf{U}_{\mathrm{art}_{k}}$ drawn from a steady distribution

$$
\mathbf{U}_{\operatorname{art}_{k}} \sim \mathcal{N}\left(\overline{\mathbf{u}}_{k \mid k-1}, \mathbf{B}_{\ell}\right)
$$

where the background covariance $\mathbf{B}_{\ell}$ is selected from a set $B=\left\{\mathbf{B}_{\ell}\right\}_{\ell=1}^{n_{B}}$ using a classification scheme as discussed in Section 3.3. We then form an augmented forecast ensemble matrix from the natural and artificial members,

$$
\mathbf{U}_{k \mid k-1} \leftarrow\left[\begin{array}{ll}
\mathbf{U}_{k \mid k-1} & \left.\mathbf{U}_{\mathrm{art}_{k}}\right]
\end{array}\right.
$$

This augmented ensemble is used in place of the natural ensemble in (2.8), providing an augmented sample covariance. This in turn provides an augmented Kalman gain in (2.12), which we apply in the analysis step. This allows the natural ensemble size to be significantly smaller than that required to statistically resolve the forecast covariance, reducing computational cost. The structure of an augmented run with $n_{B}=3$ backgrounds is illustrated in Figure 3.2.

Although the ability to update the background distribution set using forecast ensembles from an augmented run would be attractive in practice, we do not consider this here. Because the background distributions are used to form the augmented forecast ensemble and this, in turn, affects the convergence of natural ensemble members, any new training data that may 
be obtained from an augmented run is not independent of the information already present in the background set. This would introduce a potential source of bias in our statistical model. For our current implementation, generating runs and augmented runs are therefore mutually exclusive.

\subsection{Background Classification}

The classification scheme takes a deviation forecast ensemble $\tilde{\mathbf{U}}_{k \mid k-1}$, which has covariance $\mathbf{C}_{k \mid k-1}$, and finds its best match from a set of backgrounds $B$. A classification scheme is required in two scenarios. In the generating run, each forecast ensemble must be sorted among a set of backgrounds. The ensemble is then appended to the matching background ensemble. In subsequent augmented runs, the (statistically under-resolved) forecast ensemble must be matched to the appropriate statistically-resolved background. Artificial members are then sampled from this background.

This poses a statistical regime classification problem, which depends on two aspects of the generating or augmented run. The first is the particular trajectory of the ground truth, which determines the actual underlying flow regimes captured. The second is the uncertainty in the forecast ensemble, which depends on the initial conditions and the observation model. Because of this, two forecast ensembles that correspond to the same underlying state may still have very different distributions.

\subsubsection{Distance-Based Classification}

The classification problem is relatively straightforward for the generating run, as both the background and the forecast distributions are statistically resolved. In a generating run each new forecast ensemble must either be matched to the most appropriate background or made into its own new background. To sort forecast ensembles into $n_{B}$ background ensembles, we use the Wasserstein metric as the measure of "distance" between distributions. The Wasserstein metric is chosen for its stability with low-rank distributions, as it does not involve a matrix inverse. It has also been used successfully in the past in reacting flow regime classification [16]. When applied to two zero-mean Gaussian distributions with covariances $\mathbf{B}_{\ell}$ and $\mathbf{C}_{k \mid k-1}$, the Wasserstein distance $d^{2}$ is

$$
d^{2}=\operatorname{tr}\left(\mathbf{C}_{k \mid k-1}+\mathbf{B}_{\ell}-2\left(\mathbf{C}_{k \mid k-1}^{1 / 2} \mathbf{B}_{\ell} \mathbf{C}_{k \mid k-1}^{1 / 2}\right)^{1 / 2}\right)
$$

We refer to Appendix A.2 for a memory-efficient implementation of Wasserstein distance. If the background set contains fewer than $n_{B}$ distributions, the ensemble deviation matrix 
$\tilde{\mathbf{U}}_{k \mid k-1}$ with covariance $\mathbf{C}_{k \mid k-1}$ becomes its own background ensemble $\tilde{\mathbf{W}}_{\ell}$ with covariance $\mathbf{B}_{\ell}$. If the background set contains $n_{B}$ distributions, we first match $\mathbf{C}_{k \mid k-1}$ to the background $\mathbf{B}_{\ell}$ with the smallest Wasserstein distance, then we append $\tilde{\mathbf{U}}_{k \mid k-1}$ to the corresponding background ensemble $\tilde{\mathbf{W}}_{\ell}$, as per (3.3).

Additional complications arise however with the augmented run, as although the background distribution is statistically resolved, the forecast is not, and consequently the forecast covariance must be treated as a random variable. As discussed in the motivation for inflation techniques, this tends to lead to an underestimated forecast covariance. This requires us to account for the relative uncertainty in the distribution represented by each natural ensemble, therefore augmented runs may use a classification scheme modified from that used for generating runs.

To demonstrate the consequences of using the minimum Wasserstein distance criterion in the augmented run, let us consider the case where the forecast covariance $\mathbf{C}$ is a Monte Carlo approximation with $n_{\text {en }}$ samples of the identity matrix I, which is our "true" covariance. This represents a worst-case scenario for distance-based matching because the eigenvalues are all the same, so there are no relatively dominant or negligible eigenmodes. If our background set contains both the true match $\mathbf{I}$ and a scaled copy $\rho \mathbf{I}$, we wish to see whether the underresolved forecast covariance is at significant risk of falsely matching with $\rho \mathbf{I}$ for $\rho \neq 1$. The Wasserstein distance in this case can be expressed as

$$
\begin{aligned}
d^{2} & =\operatorname{tr}\left(\mathbf{C}+\rho \mathbf{I}-2\left(\mathbf{C}^{1 / 2} \rho \mathbf{I} \mathbf{C}^{1 / 2}\right)^{1 / 2}\right) \\
& =\operatorname{tr}(\mathbf{C})+\rho n_{u}-2 \rho^{1 / 2} \operatorname{tr}\left(\mathbf{C}^{1 / 2}\right) .
\end{aligned}
$$

To find the scaling $\rho$ that is most likely to trick the minimum distance criterion into picking $\rho \mathbf{I}$ over $\mathbf{I}$, we solve the minimization problem for Wasserstein distance,

$$
\frac{\mathrm{d} d^{2}}{\mathrm{~d} \rho}=n_{u}-\rho^{-1 / 2} \operatorname{tr}\left(\mathbf{C}^{1 / 2}\right)=0,
$$

which gives us the expression for $\rho$ in terms of our Monte Carlo distribution,

$$
\rho=\left(\frac{\operatorname{tr}\left(\mathbf{C}^{1 / 2}\right)}{n_{u}}\right)^{2}=\frac{\left(\sum_{j=1}^{n_{\mathrm{en}}-1} \varsigma_{j}(\tilde{\mathbf{U}})\right)^{2}}{n_{u}^{2}\left(n_{\mathrm{en}}-1\right)},
$$

where $\varsigma_{j}(\tilde{\mathbf{U}})$ is the $j$ th singular value of the forecast ensemble $\tilde{\mathbf{U}}$. Because the forecast ensemble is a random variable whose columns $\tilde{\mathbf{u}}_{j} \sim \mathcal{N}(\mathbf{0}, \mathbf{I})$, we finish by finding the expected value of $\rho$ numerically. We assume an identity matrix of size $36 \times 36$ and plot the expected 


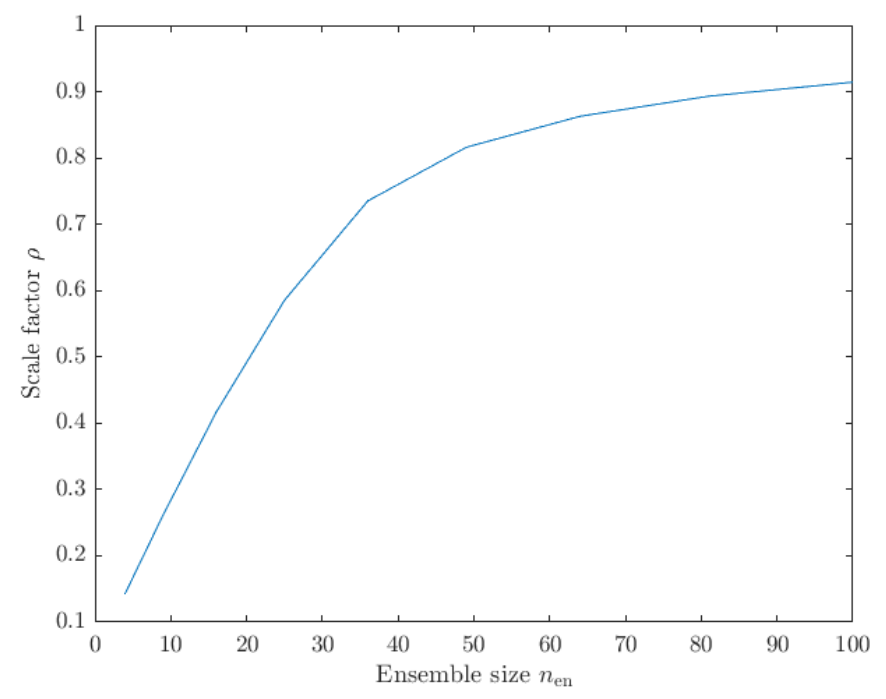

Figure 3.3: Expected value of scale factor $\rho$ that minimizes Wasserstein distance between a $36 \times 36$ identity matrix and its Monte-Carlo approximation, as a function of ensemble size.

value of $\rho$ in Figure 3.3. As we can see, though $\rho$ asymptotically approaches 1 as $n_{\mathrm{en}} \rightarrow \infty$, it is substantially smaller at most practical ensemble sizes. Even at $n_{\mathrm{en}}=n_{u}=36, \rho \approx 0.735$.

Consequently, the minimum distance criterion may falsely select an underestimated background covariance to match an undersized ensemble. This may be useful as a more "cautious" matching criterion, in the sense that it less likely to affect the performance significantly with large-covariance artificial members, behaving more like an unaugmented filter. We also however consider a matching criterion that accounts for the disparity between the highly-resolved background and the poorly-resolved forecast.

\subsubsection{Bayesian Classification}

To avoid this preference for more converged background distributions, we propose an alternative matching criterion for augmented runs. We take a Bayesian approach to estimating likelihood that a forecast ensemble $\tilde{\mathbf{U}}_{k \mid k-1}$ with $n_{\text {en }}$ ensemble members belongs to background $\mathbf{B}_{\ell}$,

$$
p\left(\mathbf{B}_{\ell} \mid d_{\ell}^{2}\right)=\frac{p\left(d_{\ell}^{2} \mid \mathbf{B}_{\ell}\right) p\left(\mathbf{B}_{\ell}\right)}{p\left(d_{\ell}^{2}\right)} \propto p\left(d_{\ell}^{2} \mid \mathbf{B}_{\ell}\right) p\left(\mathbf{B}_{\ell}\right),
$$

where $d_{\ell}^{2}$ is the distance between our forecast and the background $\mathbf{B}_{\ell}$ as calculated by (3.6). This requires us to estimate the prior probability $p\left(\mathbf{B}_{\ell}\right)$ and the likelihood $p\left(d_{\ell}^{2} \mid \mathbf{B}_{\ell}\right)$.

The backgrounds are empirical distributions and therefore random variables, however due to the large ensemble sizes used to form them, we are able to treat them as converged, statistically-resolved distributions. The only random variable that determines the marginal 


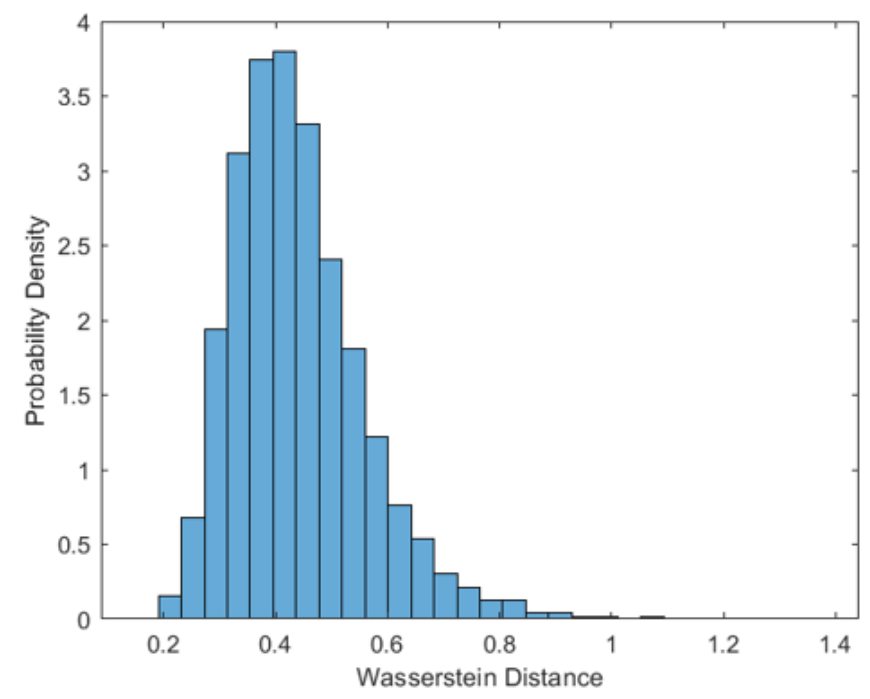

Figure 3.4: Probability density function of Wasserstein distance between background and Monte Carlo approximation.

likelihood $p\left(d_{\ell}^{2}\right)$ is therefore the forecast ensemble. This is irrelevant to the selection of a maximum likelihood background, therefore it can be neglected from our matching scheme.

The prior $p\left(\mathbf{B}_{\ell}\right)$ may be set depending on our understanding of the process model and scenario under consideration. An uninformed prior may be $p\left(\mathbf{B}_{\ell}\right)=1 / n_{B}$ for each of all $n_{B}$ backgrounds. If the background clusters are assumed to be representative of all possible regimes, the prior may be weighed by the size of each cluster, corresponding to the higher prevalence of certain regimes. The prior may also incorporate the information about the background covariance used in the previous analysis step, reflecting the natural ensemble's tendency to stay within a certain regime, and resisting rapid switching between backgrounds. For the results produced in this work, we weigh each prior by the size of the respective cluster.

The likelihood $p\left(d_{\ell}^{2} \mid \mathbf{B}_{\ell}\right)$ requires us to first resolve the distribution of expected distance $d^{2}$ assuming the forecast ensemble belongs to background $\mathbf{B}_{\ell}$. We do this via a MonteCarlo approach. We first draw $n_{\mathrm{en}}$ random members from $\mathcal{N}\left(\mathbf{0}, \mathbf{B}_{\ell}\right)$, forming an ensemble the same size as the forecast. We then find the distance between this distribution and the background. This is repeated until we resolve a distribution of distance between $\mathbf{B}_{\ell}$ and its own Monte-Carlo approximation of size $n_{\mathrm{en}}$, as in Figure 3.4. Using this distribution, we find the probability density at $d_{\ell}^{2}$ and this serves as our likelihood term $p\left(d_{\ell}^{2} \mid \mathbf{B}_{\ell}\right)$. We finally choose the background $\mathbf{B}_{\ell} \in B$ that maximizes $p\left(d_{\ell}^{2} \mid \mathbf{B}_{\ell}\right) p\left(\mathbf{B}_{\ell}\right)$.

The shape of the distribution in Figure 3.4 is not known in general. In the limit of large sample size (i.e., $n_{\mathrm{en}} \rightarrow \infty$ ), Theorem 2.1 from Rippl et al. [30] shows that the Wasserstein distance between a Gaussian distribution $\mathbb{P}$ and a Monte-Carlo approximation of size $n_{\mathrm{en}}$ of 
another Gaussian distribution $\mathbb{Q}$ itself follows a Gaussian distribution, centred on the true Wasserstein distance between $\mathbb{P}$ and $\mathbb{Q}$. The empirical behaviour converges on this limiting distribution only when $n_{\mathrm{en}} \gg n_{u}$, which is not true in this case. When considering smallensemble distribution estimates, this distribution of Monte-Carlo Wasserstein distance visibly has significant skew as shown in Figure 3.4. The algebraic manipulation of random variables in (3.6) is not straightforward, therefore we attempt to fit a known probability distribution to the sample data. Following the principle of maximum entropy, as we know that the Wasserstein distance $d^{2}>0$, we use the Gamma distribution to model the Wasserstein distance distribution. We estimate the Gamma parameters $\alpha$ and $\beta$ in terms of the mean $m_{d}$ and the variance $s_{d}^{2}$ of the sample data, according to

$$
\begin{aligned}
& \alpha=m_{d}^{2} / s_{d}^{2}, \\
& \beta=s_{d}^{2} / m_{d} .
\end{aligned}
$$

In the limit of large ensemble size, this converges on a Gaussian distribution as the variance narrows.

\section{Background Rejection}

The Bayesian matching scheme may be naturally extended to produce a criterion for accepting or rejecting the best-match background distribution. The scheme provides us with relative probability terms $p\left(d_{\ell}^{2} \mid \mathbf{B}_{\ell}\right) p\left(\mathbf{B}_{\ell}\right)$ for all $n_{B}$ backgrounds, so we can use these to evaluate whether the matching scheme is confident that the ensemble belongs to a particular background or there are multiple matches of similar quality. This may reflect that multiple backgrounds are reasonable matches, or that none are appropriate matches. Background rejection takes a cautious approach by assuming the latter, so it skips augmentation for this time step and uses the natural ensemble. This is to ensure that augmentation does no worse than the undersized ensemble that it attempts to resolve.

To turn the relative likelihood terms $p\left(d_{\ell}^{2} \mid \mathbf{B}_{\ell}\right) p\left(\mathbf{B}_{\ell}\right)$ into posterior probabilities $p\left(\mathbf{B}_{\ell} \mid d_{\ell}^{2}\right)$, we must first estimate the marginal likelihood $p\left(d_{\ell}^{2}\right)$. If we assume that the probability is 1 that $\tilde{\mathbf{U}}_{k \mid k-1}$ belongs to a background in the set $B$, then

$$
p\left(d_{\ell}^{2}\right)=p\left(d_{1}^{2} \mid \mathbf{B}_{1}\right) p\left(\mathbf{B}_{1}\right)+\cdots+p\left(d_{n_{B}}^{2} \mid \mathbf{B}_{n_{B}}\right) p\left(\mathbf{B}_{n_{B}}\right)
$$

for all $\ell=1, \ldots, n_{B}$. Using (3.10), we then calculate the posterior probability of the forecast ensemble belonging to each background.

Finally, the background rejection criterion is to apply a threshold to the posterior $p\left(\mathbf{B}_{\ell} \mid d_{\ell}^{2}\right)$ 
that corresponds to the best-match background $\mathbf{B}_{\ell}$. If the posterior falls below the threshold, augmentation is not performed. A conservative value at which to set this threshold is $50 \%$, which means that a background is only used for augmentation if it is more likely to be the correct background than not.

\subsection{Training Strategy}

The training strategy is how we design the generating run or runs such that the resulting background set captures the relevant statistical behaviour for subsequent augmented runs. We choose the generating runs such that the statistical regime space is sufficiently wellrepresented in the background set, at least as far as is necessary for the planned augmented runs.

As discussed in Section 3.3, there are two characteristics of each background in the set that must be well-matched to the forecast ensemble: the physical state that the background corresponds to, and the uncertainty in the ensemble. A generating run whose state estimate is converged about the true solution for most of its simulation time mostly captures only the low-uncertainty statistical regimes. For an augmented run whose time domain is wholly contained within the generating run's time domain, chances are that the large-uncertainty augmentation needed early in the filter run will not be well-matched by any background in the set. A preferable training strategy may be to shorten the generating run and perform more of them, capturing a range of uncertainties for the same time domain of flow behaviour.

Covariance augmentation is a machine learning technique, and therefore the training strategy is critical to the method's performance. Computational cost is high for training, so we require an intelligent sampling method that minimizes the up-front cost and makes augmentation more useful for smaller multiple-query problems. We however have not performed a detailed study of training strategy in this work, but recommend it as a focus for future research.

\subsection{Summary}

In this section we introduced covariance augmentation, a two-step process for building and drawing upon a statistical model of system behaviour. The first step is the generating run, which builds the statistical model by classifying forecast ensembles from a large-ensemble EnKF into statistical regimes. The second step is the augmented run, which again classifies forecast ensembles from an EnKF into statistical regimes, but then supplements each forecast 
ensemble with artificial members drawn from the respective regime. This is done with the aim of improving the EnKF's ability to resolve the forecast distribution.

We discussed two classification techniques: straightforward distance-based classification and Bayesian classification, the latter of which is intended exclusively for augmented runs. We briefly discussed the need for intelligent training strategies in practice. In Chapters 4 and 5, we assess the performance of augmentation and the techniques discussed in Chapter 2 . 


\section{Chapter 4}

\section{Model Test Problems}

In this section we apply our DA techniques to three synthetic model problems. We follow the procedure detailed in Section 2.1:

1. Run the process model (2.1) for the full time interval under consideration. This time history is taken as the "ground truth" reference solution $\mathbf{u}_{k}, k \in\left[1, n_{t}\right]$, that we compare the ensemble estimate against.

2. Produce the observation data synthetically by applying the observation model (2.2) to the ground truth to obtain the observations $\mathbf{v}_{k}^{\text {obs }}, k \in\left[1, n_{t}\right]$.

3. Generate an initial ensemble as per (2.3).

4. Run the ETKF from the initial ensemble along with any resolving techniques of interest.

5. Assess the error and uncertainty in the ETKF state estimate.

Because the process and observation models, which are used to generate the reference solution and observation data, are also used in the forecast and analysis steps of the filter, the filter can be considered "perfect" in that there is no inherent model deficiency.

There are two types of plots that we use to assess the filter performance: error and uncertainty. In problems with a state that is a continuous function over a domain $\Omega$, the error over time $\epsilon_{k}, k \in\left[1, n_{t}\right]$, is calculated by taking the $L^{2}$ norm over the domain $\Omega$ of the error between the ensemble mean and the reference solution,

$$
\epsilon_{k}=\sqrt{\frac{\int_{\Omega}\left(u_{k}-\bar{u}_{k \mid k}\right)^{2} d x}{\int_{\Omega} u_{k}^{2} d x}},
$$


and the uncertainty $\sigma_{k}, k \in\left[1, n_{t}\right]$, is defined analogously in terms of the variance in the distribution,

$$
\sigma_{k}=\sqrt{\frac{\int_{\Omega} \operatorname{var}\left(u_{k \mid k}\right) d x}{\int_{\Omega} u_{k}^{2} d x}} .
$$

In practice, we approximate the integrals with a quadrature rule. For the one-dimensional model problems with uniform spatial grids presented in this chapter, the error definition simplifies to

$$
\epsilon_{k}=\sqrt{\frac{\left(\mathbf{u}_{k}-\overline{\mathbf{u}}_{k \mid k}\right)^{T}\left(\mathbf{u}_{k}-\overline{\mathbf{u}}_{k \mid k}\right)}{\left(\mathbf{u}_{k}\right)^{T} \mathbf{u}_{k}}}
$$

and the uncertainty to

$$
\sigma_{k}=\sqrt{\frac{\operatorname{tr}\left(\mathbf{C}_{k \mid k}\right)}{\left(\mathbf{u}_{k}\right)^{T} \mathbf{u}_{k}}}=\sqrt{\frac{\operatorname{tr}\left(\tilde{\mathbf{U}}_{k \mid k} \tilde{\mathbf{U}}_{k \mid k}^{T}\right)}{\left(n_{\mathrm{en}}-1\right)\left(\mathbf{u}_{k}\right)^{T} \mathbf{u}_{k}}} .
$$

We take the error and uncertainty values at the final time step, $\epsilon_{n_{t}}$ and $\sigma_{n_{t}}$ respectively, to assess the DA performance.

The model problems in this chapter are chosen in part to "stress-test" the artificial resolving techniques of inflation and localization. Certain model characteristics are designed to violate the assumptions on which these techniques are based.

Recall from Section 2.5.2 that inflation can correct underestimation in the covariance "mode strength", but it cannot introduce new modes and hence cannot correct rank deficiencies. A model problem for which inflation is ill-suited is therefore one where the forecast variance terms (i.e., the diagonal of the forecast covariance matrix) rapidly approach their large-ensemble limit, whereas the cross-correlation terms remain under-resolved. The magnitudes of forecast variance terms are quantified by the uncertainty $\sigma$, which, for the model problems chosen, is only significantly underestimated for very small ensemble sizes.

As already discussed in Section 2.5.3, localization assumes a dominant correlation length above which cross-correlation terms may be suppressed. A model problem for which localization is ill-suited is therefore one where the analysis update relies heavily on long-distance correlation terms. We achieve this using sparse observation models.

When the ensemble size is sufficiently large, we can consider an ensemble filter to be "well-resolved". We identify this as the ensemble size where the error and uncertainty have reached their respective asymptotic limits. 


\subsection{Problem Definitions}

The three model problems are one-dimensional systems used to characterize the overall statistical behaviour of different methods. They are chosen to be computationally inexpensive while still reflective of significant characteristics of physical systems. These include chaotic behaviour (for all three), transfer of kinetic energy across length scales (for Lorenz 04), and either weak (for Lorenz 96) or strong (for KS equation) correlation over long distances. The $\mathrm{KS}$ equation is also a rudimentary combustion model, serving as a stepping stone between these simple model problems and full reacting flow simulation.

\subsubsection{Lorenz 96 Equation}

The Lorenz 96 (L96) equation is a system of first-order nonlinear ODEs

$$
\frac{\mathrm{d} u_{i}}{\mathrm{~d} t}=\left(u_{i+1}-u_{i-2}\right) u_{i-1}-u_{i}+F, \quad i=1, \ldots, n_{u},
$$

which was created as a toy atmospheric model [23] and is a common model problem in DA [20]. The three terms on the right hand side of (4.5) model advection, dissipation, and external forcing respectively. Total kinetic energy in the system is conserved. Following the example setup provided by Lorenz [23], we use the model parameters $n_{u}=36$ and $F=8$, which is known to produce chaotic behaviour. Increasing the forcing term $F$ will make the system more chaotic, i.e., two similar initial conditions will diverge more quickly over time.

The time interval is $[0,2]$. There is a periodic boundary condition and the initial condition is $u_{i, k=0}=F$ for all $i$ except $n_{u} / 2$, where $u_{i=n_{u} / 2, k=0}=F+0.01$. We solve this with an adaptive-order backward differentiation formula (BDF) method using a relative error tolerance of $0.1 \%$. The reference solution to this problem is shown in Figure 4.1.

We use a linear observation model $\mathbf{H}$ which consists of $n_{v}=12$ observation nodes clustered into four evenly spaced groups of three, i.e., the observed elements of the state vector are at indices $i \in\{1,2,3,10,11,12,19,20,21,28,29,30\}$. The analysis update therefore relies on local cross-correlation terms to correct the state estimate in the regions between observation nodes. We use a short observation period of $\Delta t=0.003$, which corresponds to a single integration step of (4.5).

The behaviour of the state in L96 is only weakly correlated over long distances, but strongly correlated locally. This implies that the forecast covariance will likely be diagonallydominant, i.e., the eigenvalue decay rate is low. An under-resolved sample covariance is therefore not just underestimating the magnitude of the forecast covariance, it is missing significant eigenmodes. Because inflation is a rank-preserving operation, it is not expected 


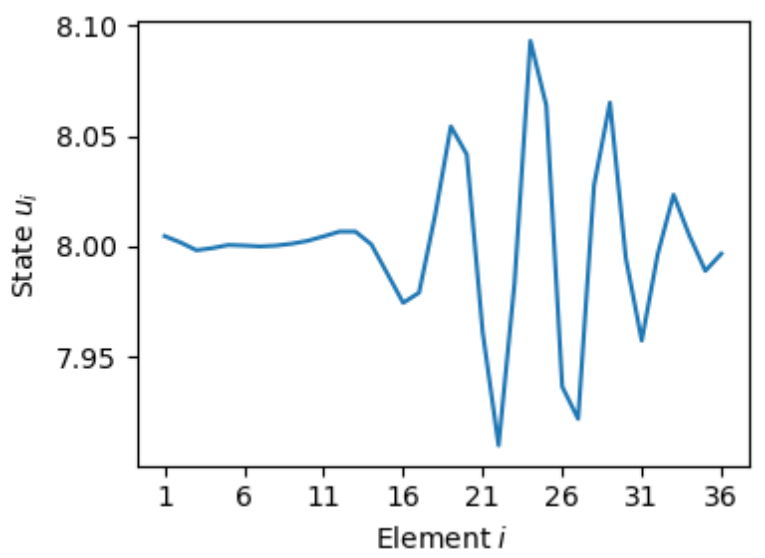

(a) Reference solution at $t=0.5$

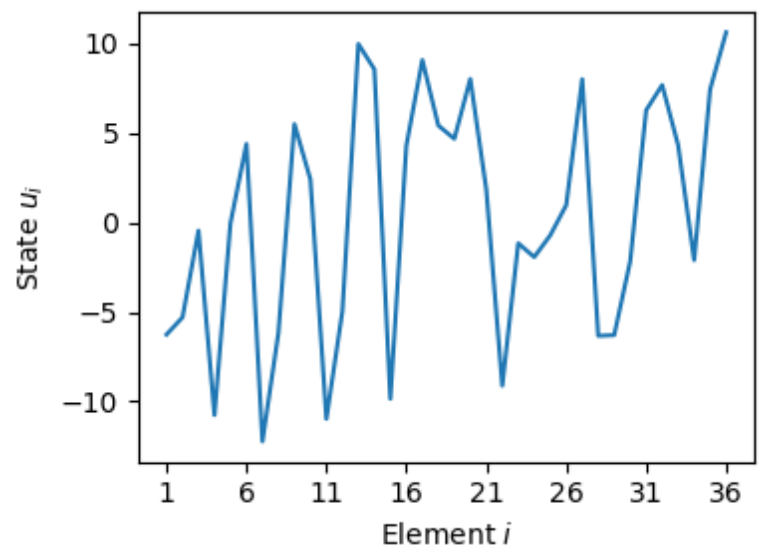

(c) Reference solution at $t=1.5$

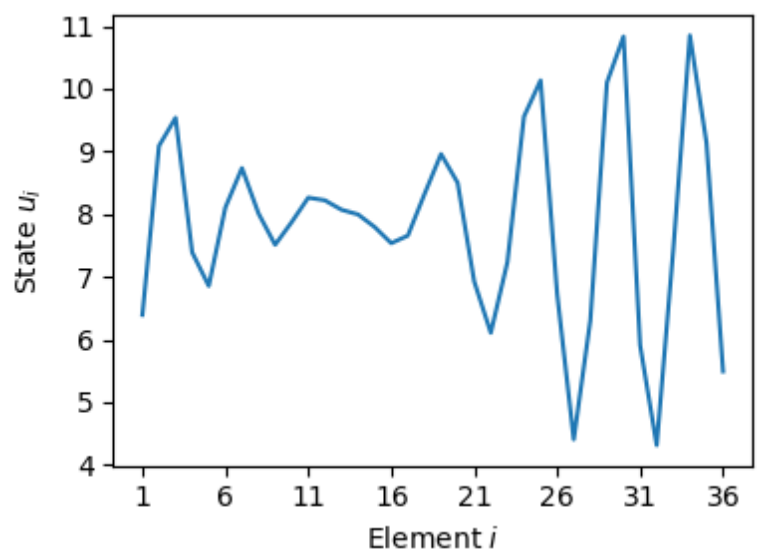

(b) Reference solution at $t=1$

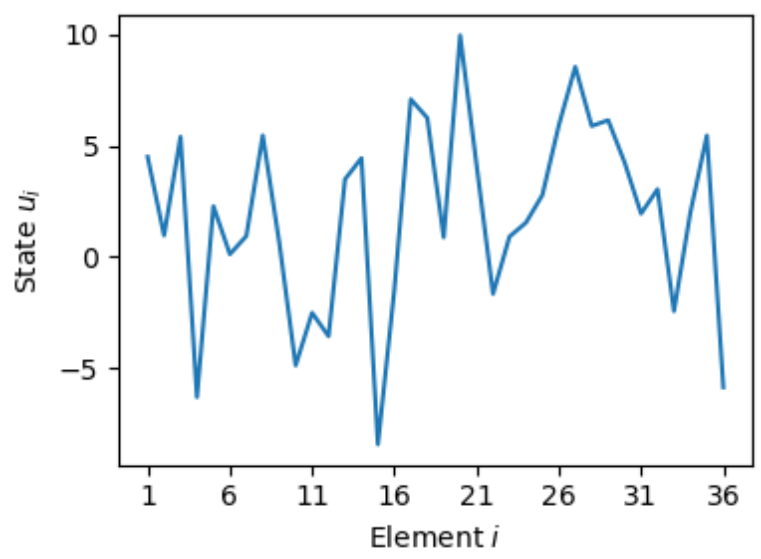

(d) Reference solution at $t=2$

Figure 4.1: Reference solution for L96 problem

to improve performance in this case. Localization however is better-suited. It assumes a dominant correlation length and suppresses long-distance correlations, which is appropriate for this model problem.

\subsubsection{Lorenz 04 Equation}

The Lorenz 04 (L04) equation is a modified form of L96 to include multiscale phenomena [22]. It is a system of first-order nonlinear ODEs

$$
\frac{\mathrm{d} u_{i}}{\mathrm{~d} t}=\left[\mathbf{u}^{l}, \mathbf{u}^{l}\right]_{K, i}+b^{2}\left[\mathbf{u}^{s}, \mathbf{u}^{s}\right]_{1, i}+c\left[\mathbf{u}^{s}, \mathbf{u}^{l}\right]_{1, i}-\mathbf{u}^{l}-b \mathbf{u}^{s}+F, \quad i=1, \ldots, n_{u},
$$


with model parameters $K, F, b$, and $c$. The terms $\mathbf{u}^{l}$ and $\mathbf{u}^{s}$ respectively contain the largescale and small-scale activity in $\mathbf{u}$ and are given by

$$
\begin{gathered}
u_{i}^{l}=\sum_{i^{\prime}=-I}^{I}{ }^{\prime}\left(\alpha-\beta\left|i^{\prime}\right|\right) u_{i+i^{\prime}}, \quad i=1, \ldots, n_{u}, \\
u_{i}^{s}=u_{i}-u_{i}^{l}, \quad i=1, \ldots, n_{u},
\end{gathered}
$$

using a modified summation $\Sigma^{\prime}$ in which the first $\left(i^{\prime}=-I\right)$ and last $\left(i^{\prime}=I\right)$ terms are scaled by $1 / 2$, and $\alpha$ and $\beta$ that control the separation of scales are given by

$$
\begin{aligned}
& \alpha=\frac{3 I^{2}+3}{2 I^{3}+4 I}, \\
& \beta=\frac{2 I^{2}+1}{I^{4}+2 I^{2}},
\end{aligned}
$$

where $I$ is an additional model parameter. Finally we define the operation

$$
[\mathbf{a}, \mathbf{b}]_{K, i}=\sum_{i^{\prime}=-J}^{J} \sum_{i^{\prime \prime}=-J}^{J}{ }^{\prime}\left(-a_{i-2 K-i^{\prime}} b_{i-K-i^{\prime \prime}}+a_{i-K+i^{\prime \prime}-i^{\prime}} b_{i+K+i^{\prime \prime}}\right) / K^{2},
$$

where we let $J=K / 2$ if $K$ is even. If $K$ is odd, we use $\Sigma$ in place of $\Sigma^{\prime}$ and let $J=(K-1) / 2$.

We slightly modify the example problem provided by Lorenz [22] to reduce the computational cost. His example uses the model parameters $n_{u}=960, F=15, I=12, K=32$, $b=10$, and $c=2.5$. Our example uses $n_{u}=480$ and $K=16$. The time interval is $[0,0.5]$. We impose a periodic boundary condition and the initial condition is

$$
u_{i, k=0}=F+5 \cos \left(\frac{2 \pi i}{n_{u}}\right)\left(1+\sin \left(\frac{2 \pi i}{n_{u}}\right)\right), \quad i=1, \ldots, n_{u},
$$

which has a length scale comparable to the size of the spatial domain. As with L96, we solve L04 with an adaptive order BDF method using a relative error tolerance of $0.1 \%$. The solution to this problem is shown in Figure 4.2.

We use a linear observation model $\mathbf{H}$ which is equal to identity $\mathbf{I}_{n_{u}}$, i.e., we take full-state observations. This is done because, when applying an ensemble filter to the L04 equation with an undersized ensemble, a sparse observation model causes the state estimate to diverge and the solver to fail. As we aim to apply artificial resolving techniques to an undersized ensemble and demonstrate their performance, it is important that we have stable baseline results to compare against. The observation period, as with L96, is $\Delta t=0.003$, which corresponds to a single integration step of (4.6).

Although this observation model was chosen for numerical stability, it provides a useful 


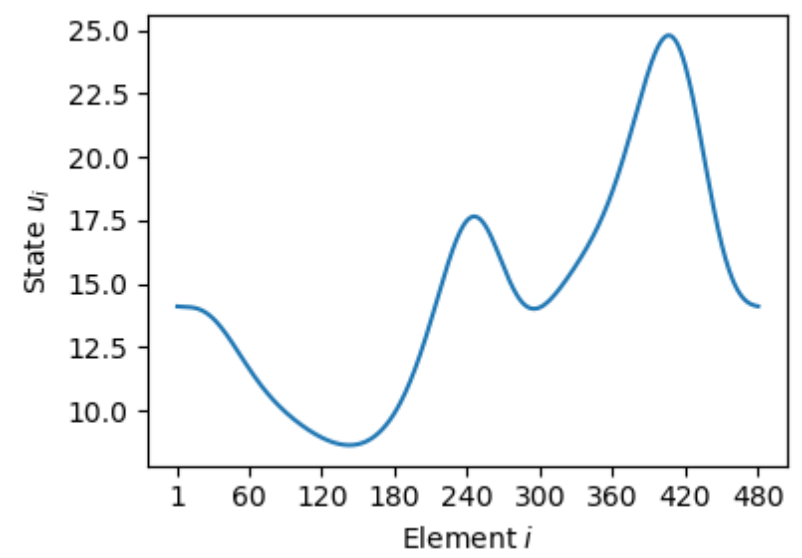

(a) Reference solution at $t=0.125$

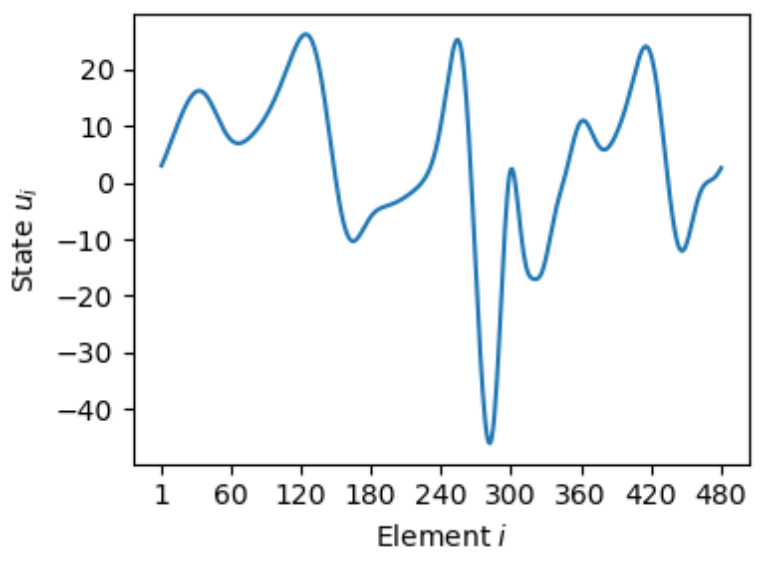

(c) Reference solution at $t=0.375$

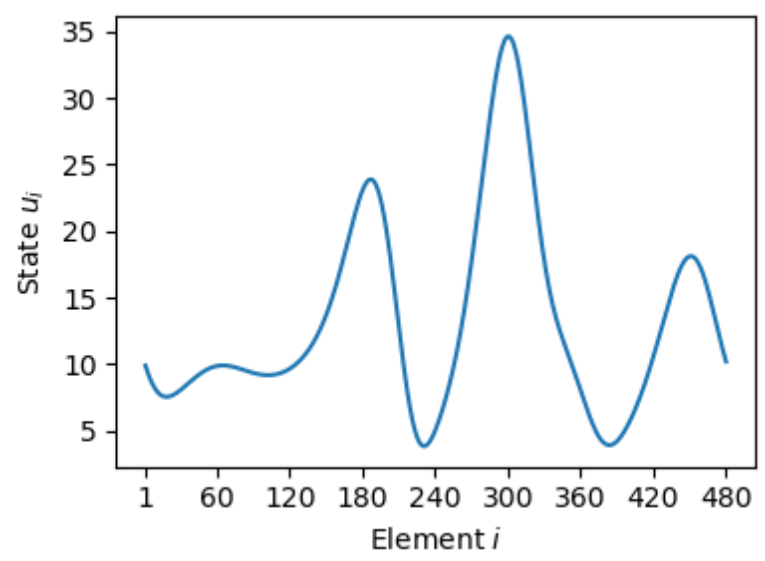

(b) Reference solution at $t=0.25$

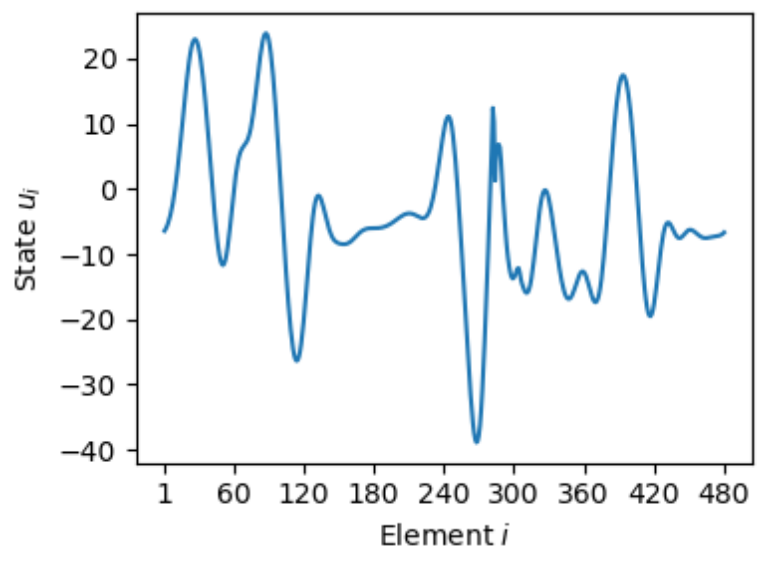

(d) Reference solution at $t=0.5$

Figure 4.2: Reference solution for L04 problem

example of a case reasonably well-suited to both inflation and localization. This is a model with continuous large-scale activity in $\mathbf{u}^{l}$ and discrete small-scale activity in $\mathbf{u}^{s}$. This leads to a range of relevant length scales, producing a covariance more balanced between long-distance cross-correlation terms and diagonal terms. Like with L96, the ensemble size required to capture this covariance therefore scales with the state size. The use of a full-state observation model however makes the cross-correlation terms less relevant to the analysis update. If they are suppressed by localization, then the analysis step may still correct every element of the state vector using the corresponding observation element, ignoring correlations over distance. Inflation has a similar effect, increasing uncertainty and leading the analysis update to rely more on the comprehensive observation data. We expect L04 to see improved performance from both artificial resolving techniques. 


\subsubsection{KS Equation}

The Kuramoto-Sivashinsky (KS) equation is a fourth-order nonlinear PDE

$$
\frac{\partial u}{\partial t}=-\frac{\partial^{4} u}{\partial x^{4}}-\frac{\partial^{2} u}{\partial x^{2}}-u \frac{\partial u}{\partial x}
$$

which models instabilities in laminar flame fronts and exhibits chaotic behaviour [32, 17]. We consider the time interval $[0,60]$ and the spatial domain $[0,16 \pi]$. There is a periodic boundary condition and the initial condition is

$$
u(x, t=0)=5 \cos \left(\frac{x}{8}\right)\left(1+\sin \left(\frac{x}{8}\right)\right) .
$$

The KS equation is discretized using second-order centered difference approximations for the spatial derivatives,

$$
\frac{\mathrm{d} u_{i}}{\mathrm{~d} t}=-\frac{u_{i-2}-4 u_{i-1}+6 u_{i}-4 u_{i+1}+u_{i+2}}{\Delta x^{4}}-\frac{u_{i-1}-2 u_{i}+u_{i+1}}{\Delta x^{2}}-u_{i} \frac{-u_{i-1}+u_{i+1}}{2 \Delta x},
$$

which we then solve with an adaptive-order BDF method. As with L96 and L04, we use a relative error tolerance of $0.1 \%$. The spatial domain is discretized into $n_{u}=128$ nodes, so $\Delta x=\pi / 8$ and $i=1, \ldots, 128$.

The ground truth reference solution is presented in Figure 4.3. After an initial transient period, KS settles into a steady behaviour with "source" and "sink" terms roughly at $x=12 \pi$ (i.e., $i=96$ ) and at $x=4 \pi$ (i.e., $i=32$ ) respectively. The waves have a mostly uniform wavelength and velocity. This leads to significant long-distance correlation over the state.

As with L96, a linear observation model is used with $n_{v}=12$ observation nodes clustered into four evenly spaced groups of three, i.e., the observed elements of the state vector are at indices $\mathbf{u} \in\{1,2,3,33,34,35,65,66,67,97,98,99\}$. For this larger state size, this is a relatively sparse observation model. The analysis step therefore relies on resolved crosscorrelation terms to correct the state estimate in between observation nodes. This is a worstcase scenario for localization, as long-distance correlations are essential to the performance of the filter. The observation period is $\Delta t=0.3$, which as with L96 and L04, corresponds to one integration step of (4.11).

Because this is the only fully continuous model problem considered in this chapter, it consequently is the only model problem where the behaviour ought not to change significantly with different values of $n_{u}$. Some practical physical problems are like this; the grid resolution (and thus state size) is set to the level necessary to capture the dynamics of the system. Beyond this minimal resolution, the dynamics do not fundamentally change. The ensemble 


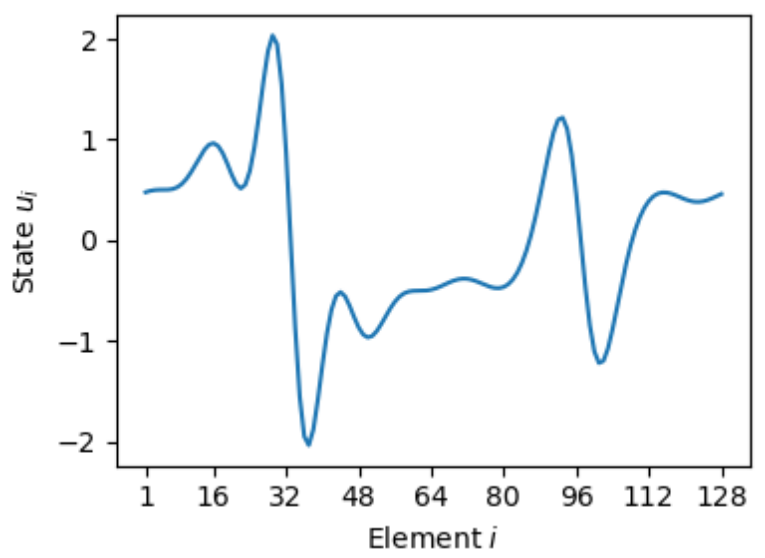

(a) Reference solution at $t=15$

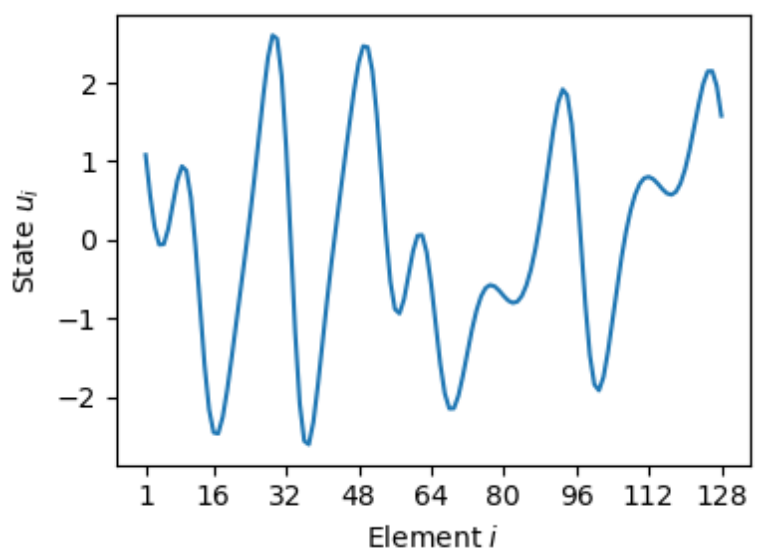

(c) Reference solution at $t=45$

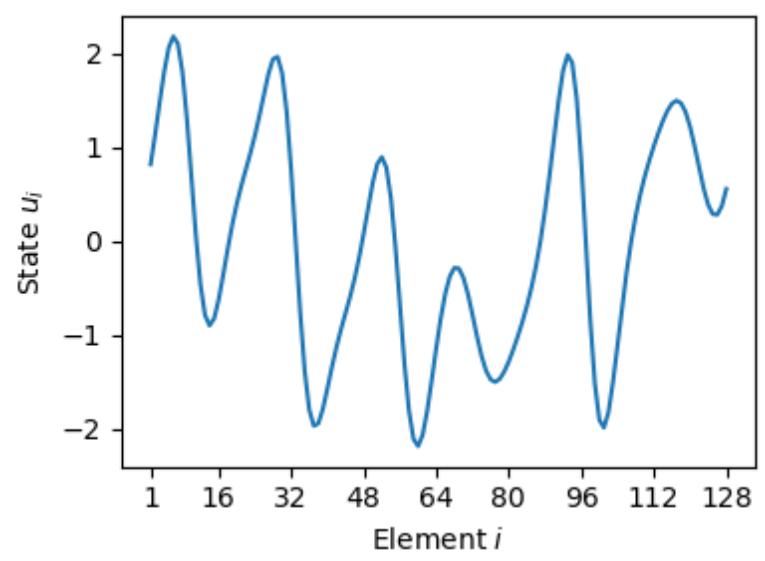

(b) Reference solution at $t=30$

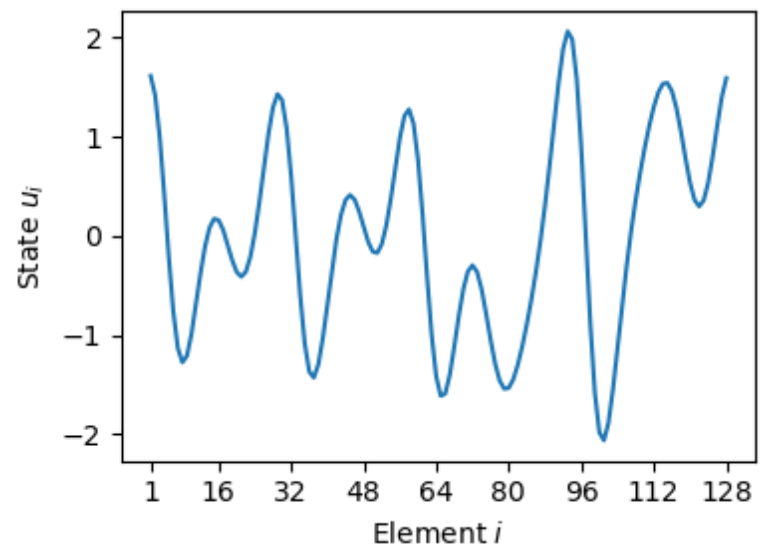

(d) Reference solution at $t=60$

Figure 4.3: Reference solution for KS problem

size is set in this same way, large enough to capture the dynamics. Ensemble size in KS is therefore decoupled from state size, which is not the case for L96 or L04.

Low Reynolds number flows may behave in this same way. Where the grid resolution required to capture the dynamics is reasonable for most computers, the ensemble size is then entirely determined by the dynamics. For LES however, this is not the case. The scale of resolved modes is by definition determined by the grid scale, so the captured dynamics, and thus ensemble size, depend on the grid scale.

\subsection{Baseline Performance}

To find the baseline performance for a statistically-resolved ETKF, we apply the ETKF to each of the three model problems described above. We use a wide range of ensemble 
sizes appropriate to each model problem to characterize the ETKF performance. We then note the required ensemble size for each model to reach the asymptotic limits for error and uncertainty.

There are two aspects to the performance of an ETKF: first there is its ability to converge from the initial ensemble toward the underlying reference trajectory, and second there is its ability to "track" the trajectory once converged. For the initial convergence, the ensemble mean is not reflective of the true solution and the covariance is very large, so the filter relies on the observation and the cross-correlation terms in the covariance to converge the ensemble toward the true solution. Once converged, the filter needs to continually correct the state estimate if it begins to drift away from the true solution. This drift will occur as a consequence of using chaotic model problems; by definition, slightly different initial conditions can develop into completely different states over a long enough time period. The ensemble must capture this drift over time in its members, which would increase the covariance. This then leads to greater correction from observation in the analysis step.

We take a moment now to discuss the plots used to present the results. The plots of error and uncertainty over ensemble size are plots of probability distributions as functions of a discrete independent variable, i.e., plots of $p\left(\epsilon \mid n_{\mathrm{en}}\right)$ and $p\left(\sigma \mid n_{\mathrm{en}}\right)$ over $n_{\mathrm{en}}$. An example is Figure 4.4, which shows the baseline performance of an ETKF as applied to Lorenz 96, Lorenz 04 , and the KS equation. The error and uncertainty are taken at the last time step of the filter run, i.e., $\epsilon=\epsilon_{n_{t}}$ and $\sigma=\sigma_{n_{t}}$. At each ensemble size, the ETKF is run repeatedly with different initial ensembles until empirical distributions of error and uncertainty are statistically converged. We consider the distributions to be converged when the variance in the sample mean of the $\log$ (error) is below a user-defined threshold of 0.003 . The trace in each plot represents the mean of all ETKF runs at a given ensemble size. The shaded colourbars represent the probability density function, as found by kernel density estimation. Note also how the $x$-axis of these plots is scaled; this is because estimating the mean of a normal distribution using the sample mean has a standard error that decreases with $\sqrt{n_{\mathrm{en}}}$.

\subsubsection{Lorenz 96}

The baseline results for an ETKF applied to L96 are shown in Figures 4.4a and 4.4b. The slow eigenvalue decay rate in the covariance is evident from the fact that, to reach asymptotic performance, the ensemble size is large relative to the state size. The error and uncertainty both remain unimodal over all ensemble sizes, suggesting that unlike what we see with KS, there is no discrete jump where the ensemble "snaps" to tracking the correct solution. This is a problem where tracking the reference solution is extremely difficult even once the ensemble 


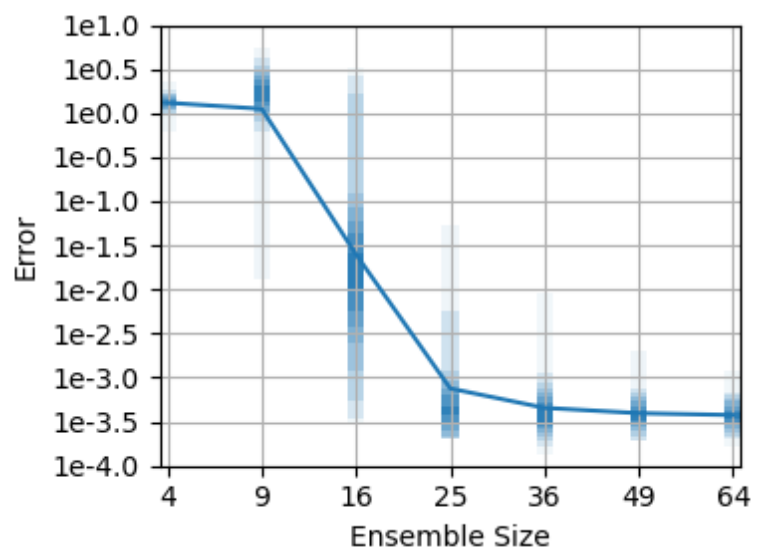

(a) L96 Error

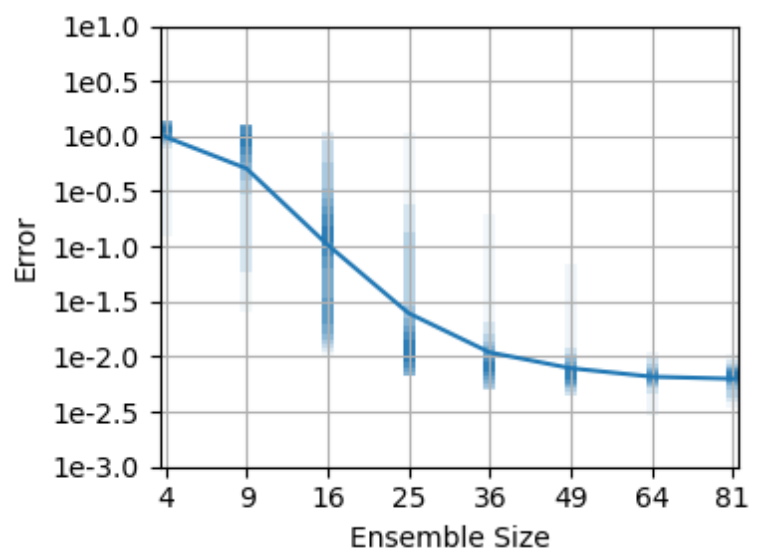

(c) L04 Error

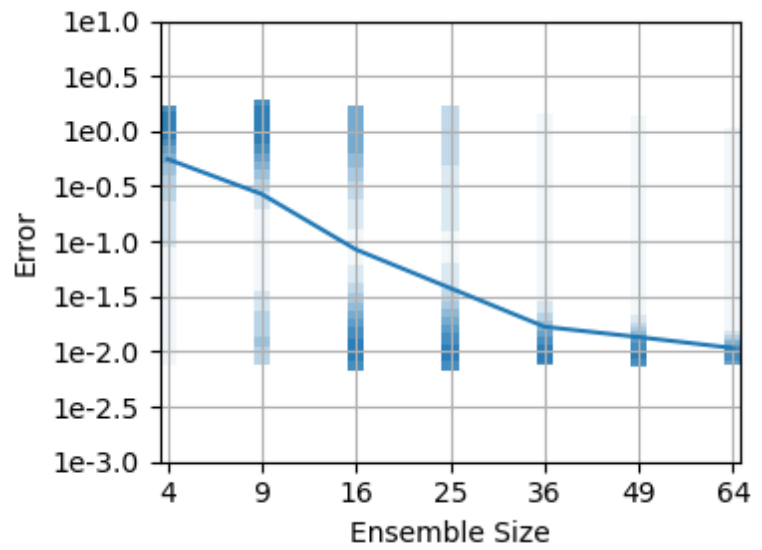

(e) KS Error

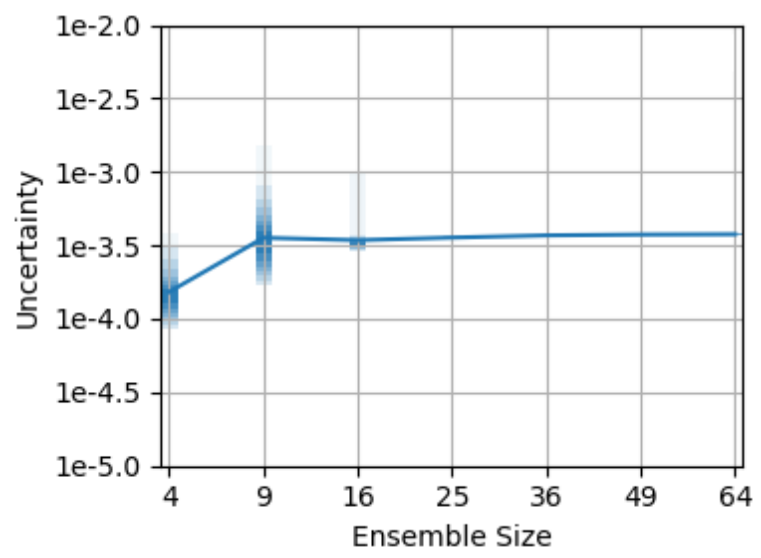

(b) L96 Uncertainty

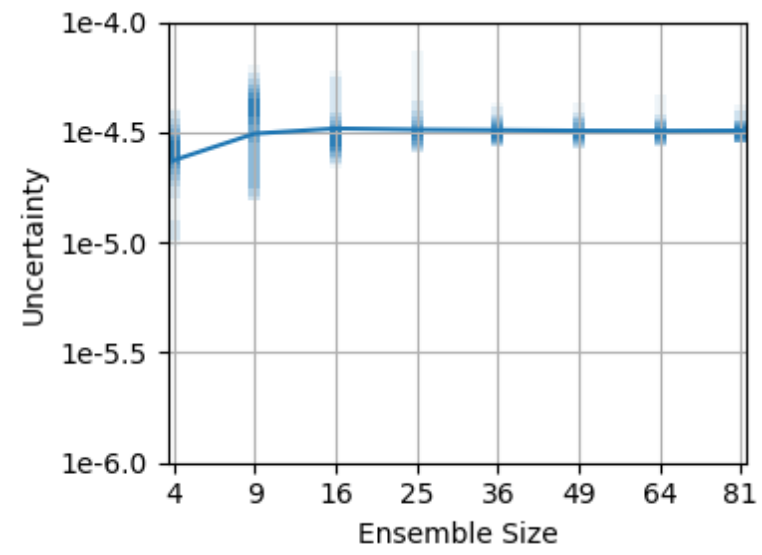

(d) L04 Uncertainty

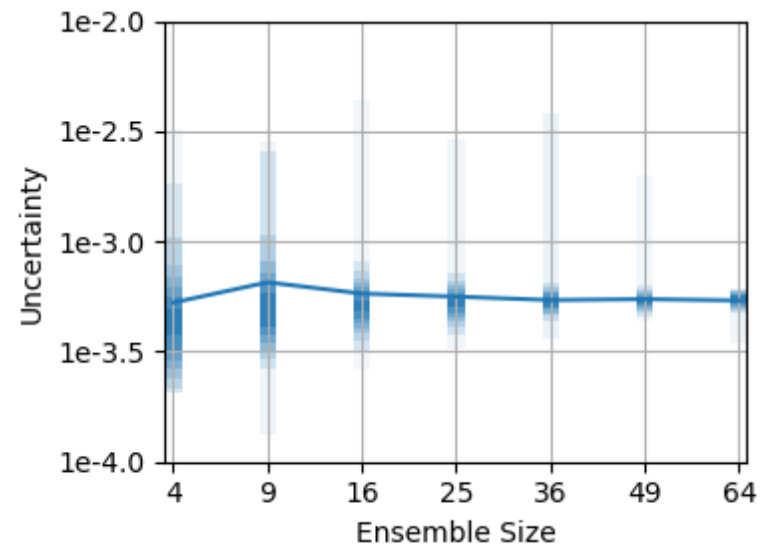

(f) KS Uncertainty

Figure 4.4: Distributions of error and uncertainty for the ETKF applied to model problems 
has converged on it.

The uncertainty is underestimated only at the very small ensemble size of 4 , however the mean uncertainty very quickly reaches the asymptotic value of $10^{-3.4}$ around ensemble size 9 . At this ensemble size the error only begins to decrease, continuing until it reaches the same limiting value of $10^{-3.4}$ around ensemble size 36 . This shows that resolving the distribution requires much more than spreading the ensemble members, suggesting that inflation will be a weak improvement if it is at all. For L96, we consider the ETKF "well-resolved" at ensemble size 64 .

\subsubsection{Lorenz 04}

The baseline results for an ETKF applied to L04 are shown in Figures 4.4c and 4.4d. Many of the characteristics we noted with L96 are also true of L04. Error and uncertainty remain mostly unimodal over all ensemble sizes, suggesting that the difficulty lies in tracking the correct solution even once converged. Uncertainty is again underestimated at the smallest ensemble sizes, though it increases quickly to the asymptotic value of $10^{-4.5}$ by ensemble size 16. The error only reaches its asymptotic limit of $10^{-2.2}$ by ensemble size 64 , which is where we consider the filter "well-resolved."

\subsubsection{KS Equation}

The baseline results for an ETKF applied to the KS equation are shown in Figures 4.4e and 4.4f. Uncertainty in this case maintains a relatively consistent mean around $10^{-3.25}$, though the spread converges with larger ensemble size. The error mean again decreases with increasing ensemble size, however unlike L96 and L04 the performace distribution is almost exclusively bimodal. The modes do not shift with increasing ensemble size, but the weight shifts from the unconverged mode to the converged mode. The same bimodal distribution is not present in the uncertainty however, suggesting that the ensemble converges regardless of whether it is tracking the true solution. The larger ensemble size allows it to more reliably make the initial step from unconverged ensemble to tracking the true solution. This shift is mostly made between ensemble size 25 and 36 . The performance continues to improve with larger ensemble size, however now the spread in the converged mode narrows as tracking becomes more reliable. The error approaches an asymptotic value of $10^{-2}$ around ensemble size 64, which is the ensemble size where we consider the ETKF "well-resolved." 


\subsection{Inflation}

The results for covariance inflation are presented in Figure 4.5. We saw in the baseline results in Figure 4.4 that the uncertainty approaches its large-ensemble limit very quickly in all three model problems. Inflation therefore can only overestimate the forecast covariance, which we see in Figure 4.5. Consequently, the effect of inflation on error in L96 and KS is extremely limited. In the case of L96, the adaptive inflation scheme increases the uncertainty by almost an order of magnitude, suggesting that the calculated $\rho$ is far too large. In the case of $\mathrm{KS}$, the inflation is more reasonable, however the improvement to performance is extremely slight regardless. It is clear from the uncertainty plots that underestimated covariance (i.e., underestimated mode strength) is not a significant concern for most ensemble sizes in these model problems, therefore inflation is not an appropriate method to improve performance.

There are scenarios using L96 however where inflation is an effective technique. An example is Ahmed et al. [2], who used a denser observation model of $n_{v}=18$ evenly-spaced nodes and a fixed, modest inflation factor $\rho=1.04$. With this observation model, each node was either directly observed or adjacent to a node that was. This reduced the dependence on potentially under-resolved cross-correlation terms. An inflated forecast covariance is therefore less likely to cause the state estimate to diverge, and this is reflected in the improved performance they achieve.

For L04 there is indeed some improvement to error, roughly by a factor of two for most ensemble sizes. The best-case performance at large ensemble size is also better than in the uninflated case. This is despite the fact that inflated uncertainty is higher than the large-ensemble uninflated uncertainty. This is a consequence of taking full-state observations. Increased uncertainty causes the analysis update to favour the observation data when correcting the state estimate. Since the observation model fully determines the state, this reduces the error.

In model problems where small ensemble size does not significantly underestimate the forecast covariance, we saw that there is little to gain from applying inflation. For L96 and KS, the filter performance depends primarily on its ability to resolve cross-correlation terms in the covariance, which inflation is not intended to address. For L04 however, we see how an appropriate model problem is helped by inflation. It prevents "overconfidence" in the state estimate, making it more open to correction by observation data. 


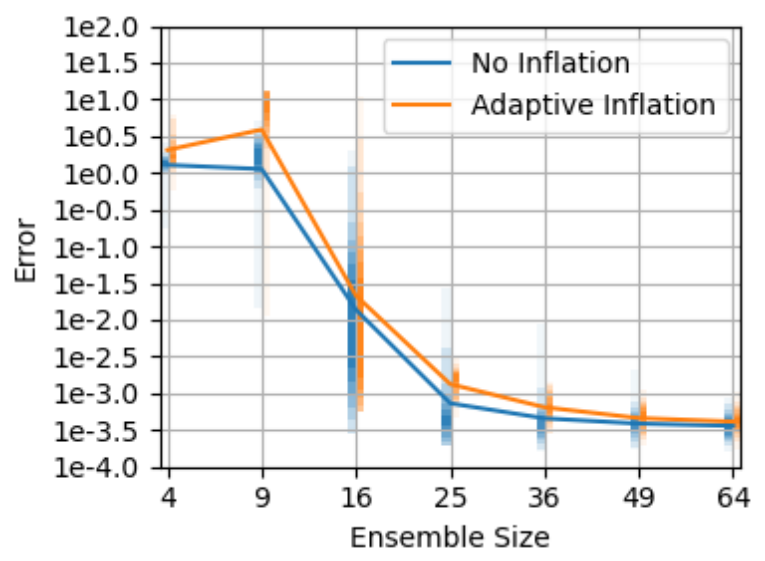

(a) L96 Error

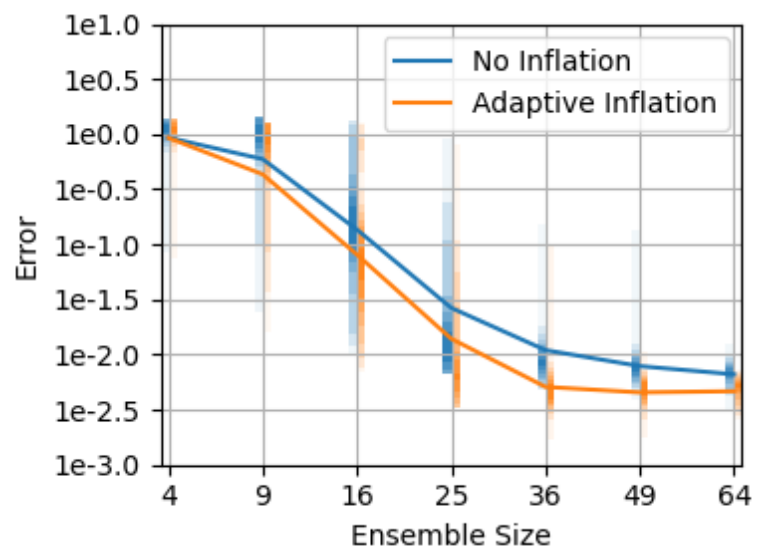

(c) L04 Error

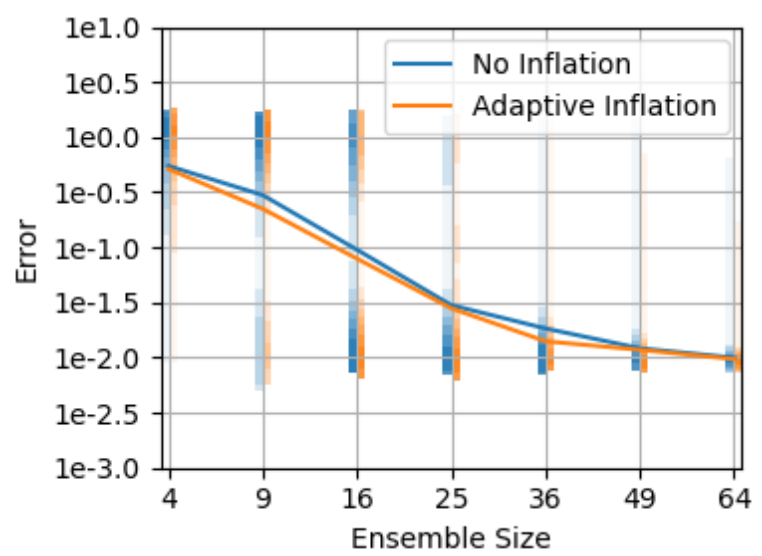

(e) KS Error

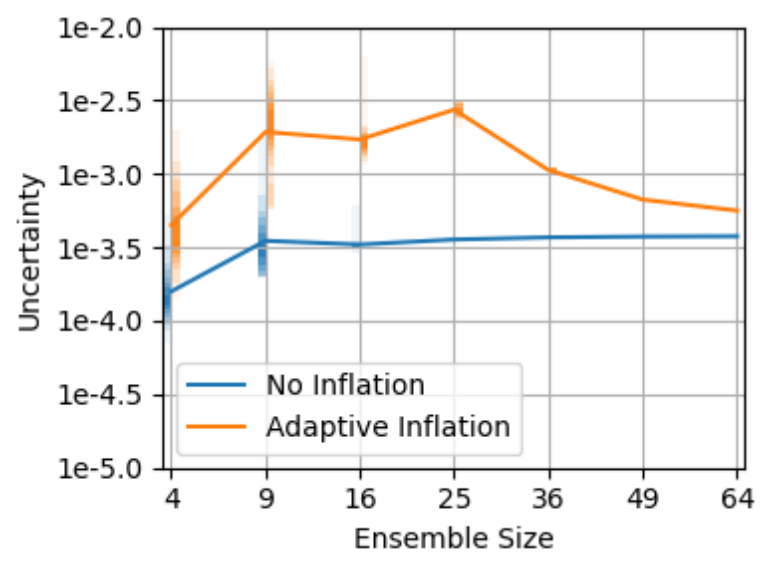

(b) L96 Uncertainty

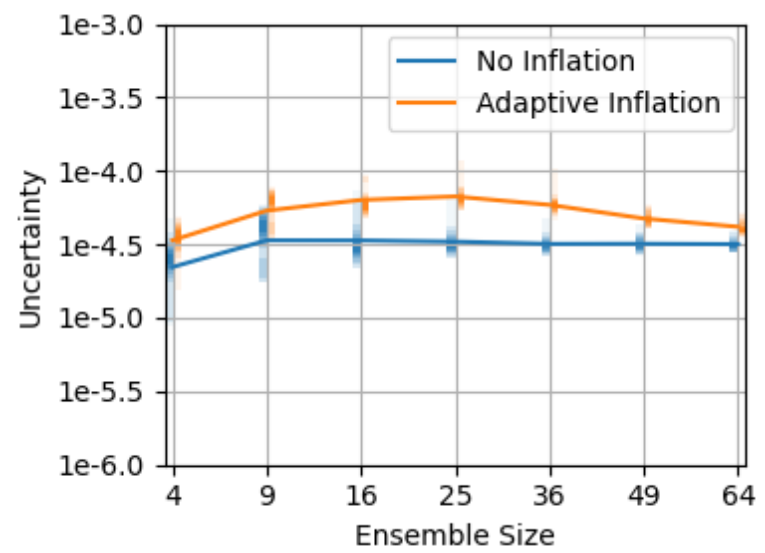

(d) L04 Uncertainty

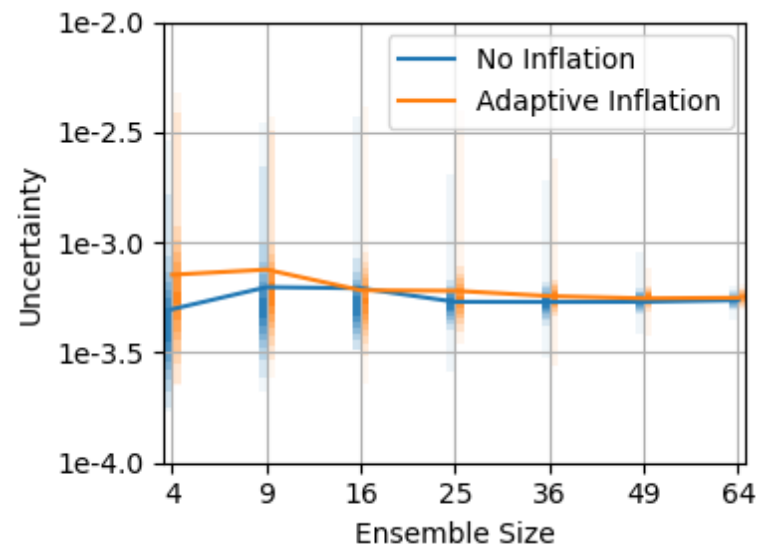

(f) KS Uncertainty

Figure 4.5: Distributions of error and uncertainty for the ETKF with inflation applied to model problems 


\subsection{Localization}

The results for observation localization are presented in Figure 4.6. We empirically selected appropriate length scales for each model problem. Because the elements of the L96 and L04 state vector correspond to discrete nodes, we select an integer length scale from the set $\{4,5, \ldots, 16\}$. For KS, we search the set $\{2 \pi, 4 \pi, 8 \pi, 16 \pi, 32 \pi\}$, however no length scale yields improved performance.

For L96 we use a length scale of 7 nodes, localization provides a clear improvement over the unaugmented filter for small ensemble sizes. This is consistent with the fact that the covariance for L96 tends to be more diagonal than KS or L04, so localization brings the sample covariance closer to the well-resolved limit. This is consistent with the known performance of a localized EnKF with L96 [4]. In Figure 4.6a, we see that where the unmodified filter performs well, localization provides little improvement. For ensemble size 16 however, the performance of the unmodified filter is spread over four orders of magnitude. Localization improves the mean performance significantly and controls the spread, making the filter perform more reliably when the ensemble is moderately undersized.

For L04 we use a length scale of 10 nodes, which leads to significant performance improvement at small ensemble sizes. Localization is more easily applicable in cases where the observation data is spatially dense, as cross-correlation terms are less essential in applying the analysis update. In this case, we take full-state observation data, which is as dense as possible. Localization therefore improves the filter performance by reducing the effect of poorly-resolved cross-correlation terms and forcing each state variable to more directly follow the observation data.

For KS however, recall from Figure 2.1 and the discussion in Section 4.1.3 that there are significant cross-correlation terms that would be suppressed by localization, and because the observation data from KS is extremely sparse, accurately resolving these is essential to the analysis update. Localization in the case of $\mathrm{KS}$ therefore compromises performance for any effective length scale and underestimates uncertainty. For the results in Figures 4.6e and 4.6f, we used a large length scale of $L=32 \pi$ to minimize localization's effect on performance. All smaller length scales only lead to larger error.

The improvements made to L96 and L04 are consistently at small ensemble sizes. As discussed in Section 2.5.3, at larger ensemble sizes, localization can only suppress crosscorrelation terms that are not spurious. For L96 long-distance correlation terms are not a factor, so localization performance converges on the same asymptotic limit as the nonlocalized filter. For L04 however there are legitimate terms being suppressed, so the localized filter converges on a slightly higher error level. This is not an intended use case for localization 


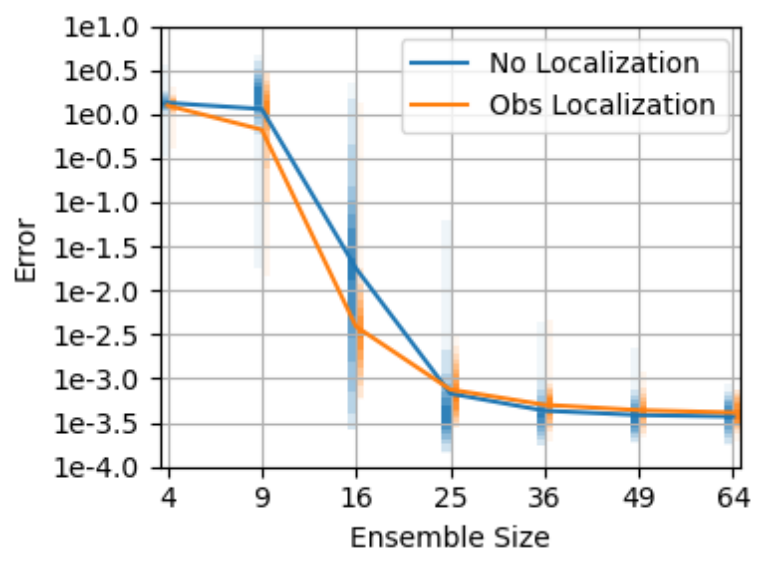

(a) L96 Error

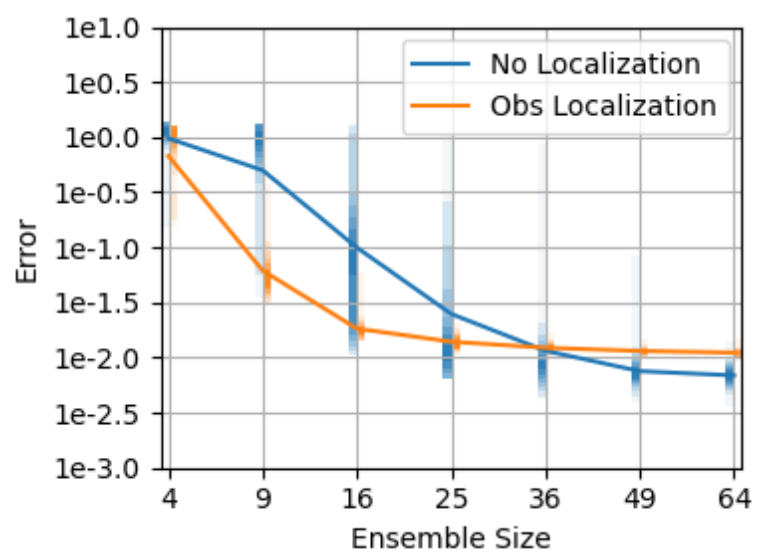

(c) L04 Error

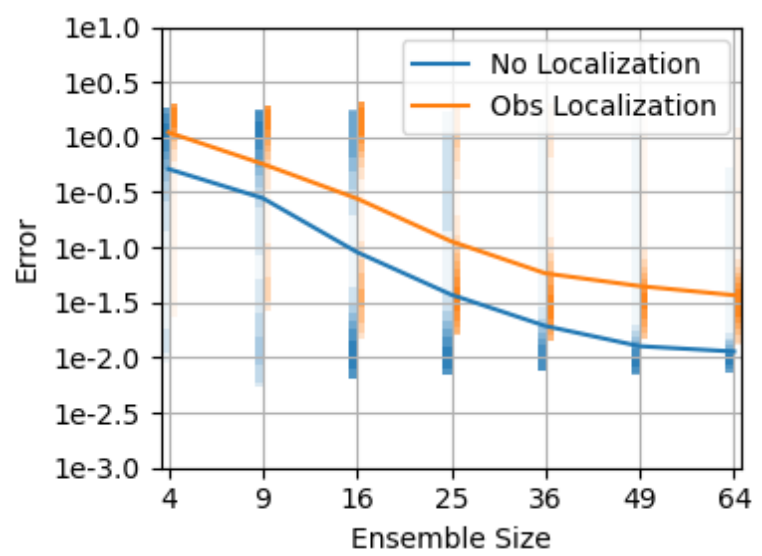

(e) KS Error

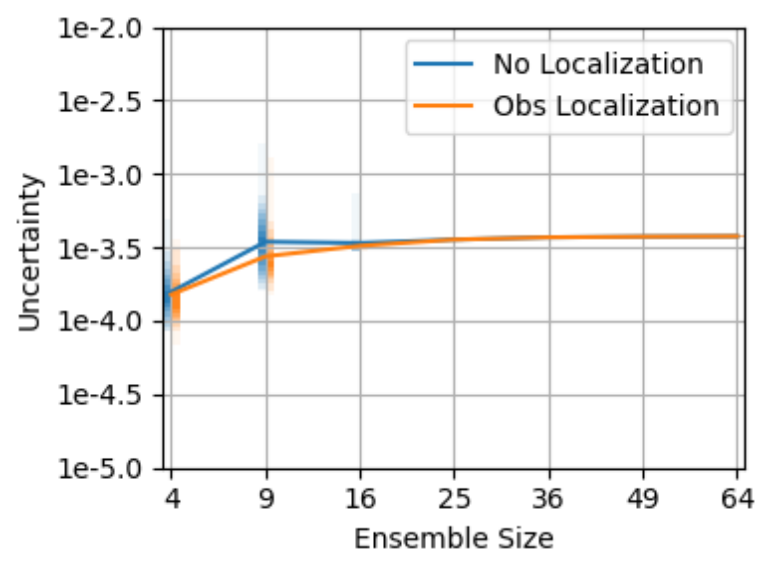

(b) L96 Uncertainty

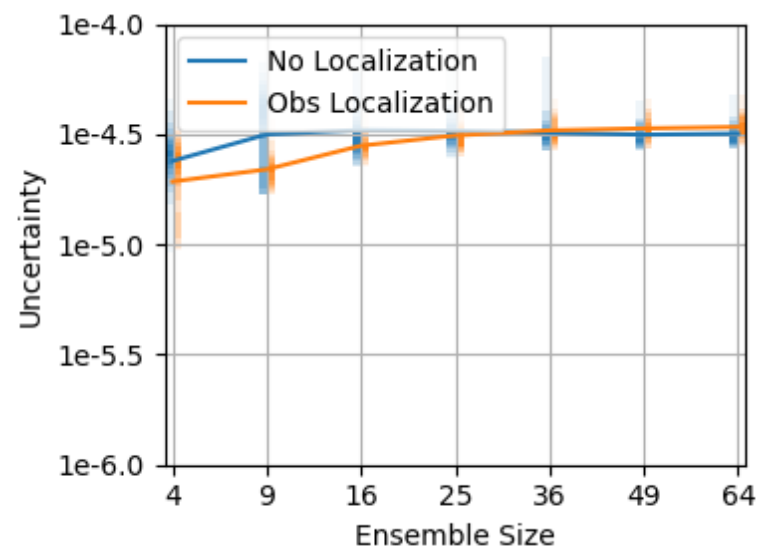

(d) L04 Uncertainty

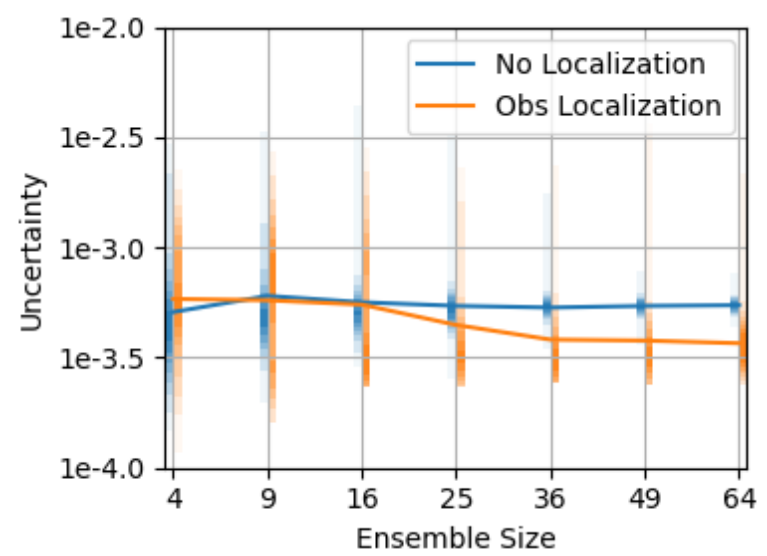

(f) KS Uncertainty

Figure 4.6: Distributions of error and uncertainty for the ETKF with localization applied to model problems 


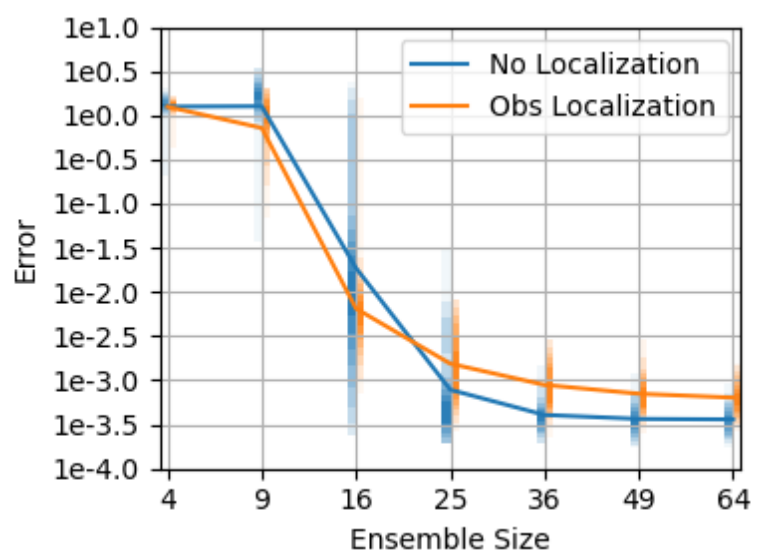

(a) L96 Error

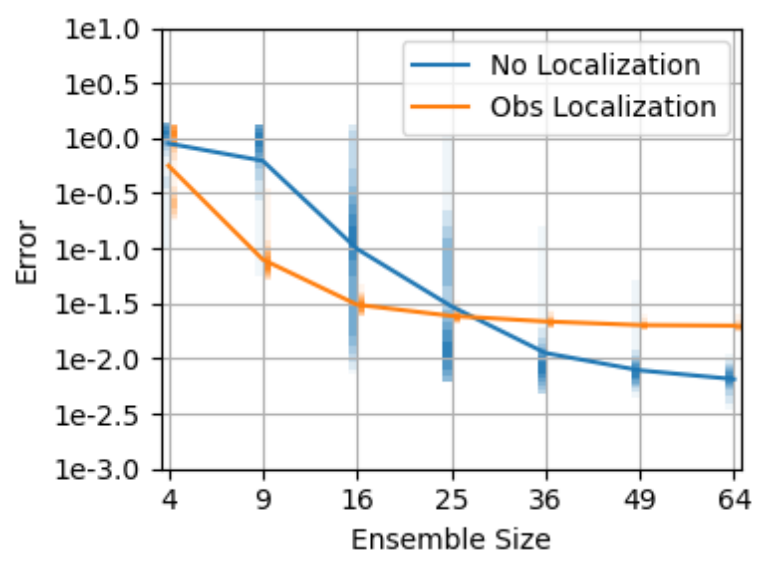

(c) L04 Error

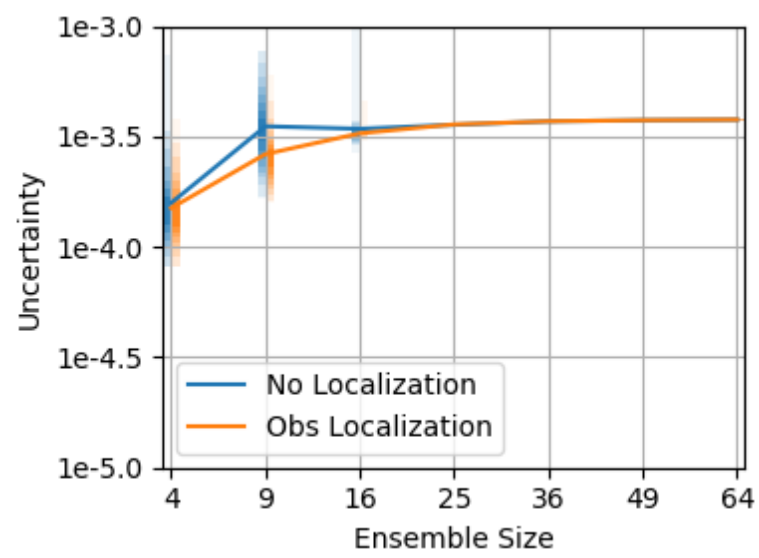

(b) L96 Uncertainty

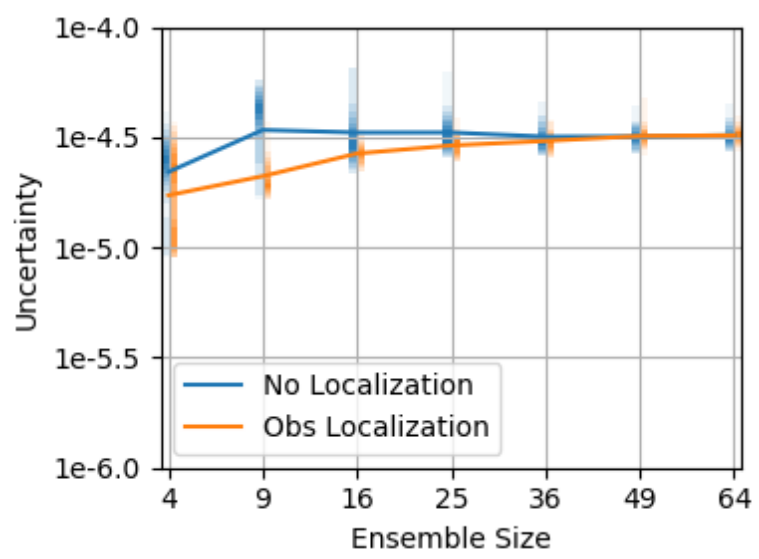

(d) L04 Uncertainty

Figure 4.7: Distributions of error and uncertainty for the ETKF with perturbed length scale localization applied to model problems

though. Localization is intended to aid with resolving small-ensemble forecast covariances, and where long-distance correlation terms are not critical to the filter performance, it helps as intended.

We briefly consider how sensitive the filter performance is to the choice of length scale. Only L96 and L04 are considered here, as we know that localization is not appropriate for our KS setup at any length scale. The results are shown in Figure 4.7.

L96 has a dominant length scale, and consequently benefits significantly from localization in the best case. Moderate changes to the length scale however significantly compromise the performance, as can be seen in Figures $4.7 \mathrm{a}$ and $4.7 \mathrm{~b}$. We have decreased the length scale from 7 nodes to 5 nodes, increasing the error by a factor of two and raising the asymptotic error level. This indicates that some useful correlation terms are being suppressed inappropriately, 
which adversely affects performance.

L04 has a range of dominant length scales, however we see in Figures 4.7c and 4.7d that, for small ensemble sizes, localization is less affected by a change in length scale from 10 nodes to 7 nodes. This is for the same reason that localization had such a strong effect to begin with: the fact that we take full-state observations makes cross-correlation terms less relevant, so suppressing them does not compromise performance as much as it would with a more limited observation model. At larger ensemble sizes however, the difference in asymptotic performance becomes apparent.

\subsection{Augmentation}

There are three scenarios we consider when applying augmentation to our test problems. First is the reproduction scenario, where the model trajectory in the generating run is the same as that in the augmented run. The second is a scenario with these trajectories differ, testing the applicability of the background set outside of the training data. The third is a scenario where the model parameters have changed between the generating and augmented runs, testing the applicability of the background set to slightly different governing equations.

For all three model problems, we produce $n_{B}=50$ backgrounds from two generating runs, each at the respective well-resolved ensemble size. We use the distance-based classification scheme described in Section 3.3.1 for both the generating and augmented runs.

\subsubsection{Reproduction Scenario}

The reproduction scenario represents the best-case scenario for applying augmentation, as the training data and test data match as closely as possible. It does not reflect a practical use case, as it is necessarily more costly than simply running a well-resolved ETKF. Nonetheless, it is useful to provide an upper bound on performance. The results are shown in Figure 4.8.

Because we know that there exist backgrounds in the set $B$ that correspond almost perfectly with this trajectory, the test is ultimately of the classification scheme and the level of clustering, i.e., the background set size $n_{B}$ relative to the number of forecast ensembles produced by generating runs. As expected, the error and uncertainty rapidly approach their respective asymptotic limits. There is still some dependence on ensemble size, but the minimum is reached much faster than with an unaugmented ETKF. The limiting factor determining ensemble size is no longer whether the covariance is well-resolved, but whether the classification scheme has enough information to select the appropriate background covariance. Once a sufficiently comprehensive set of backgrounds is collected, this can reduce 


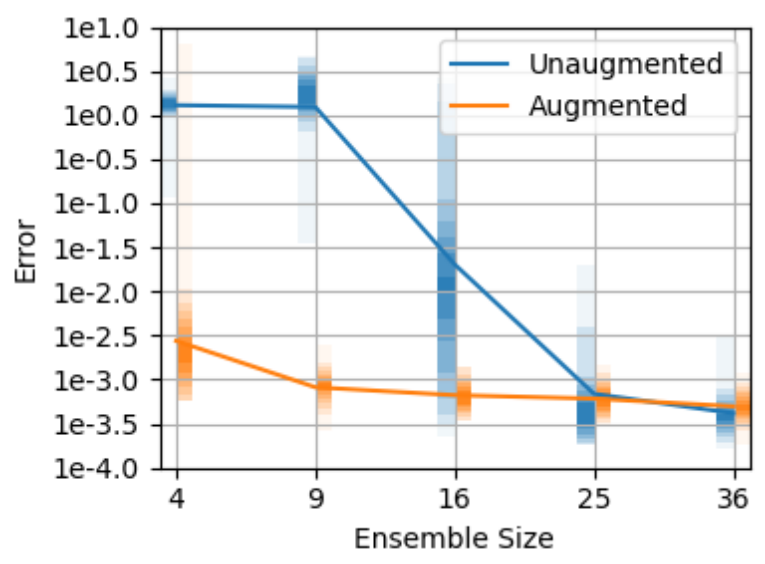

(a) L96 Error

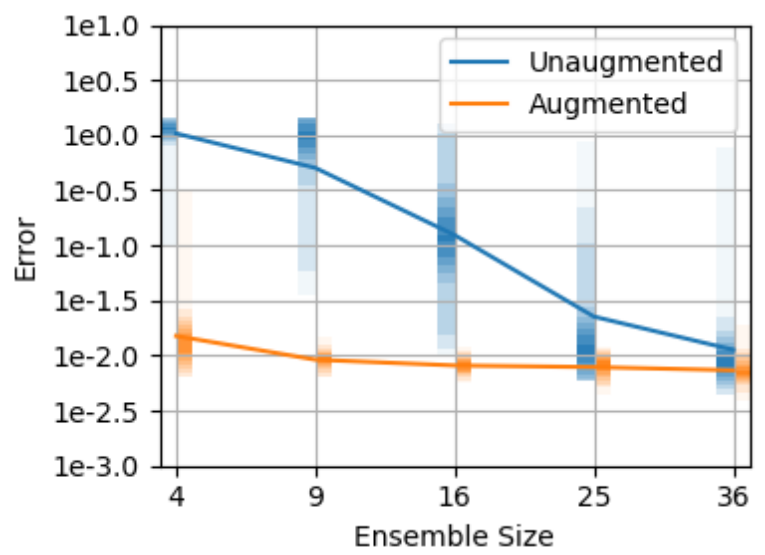

(c) L04 Error

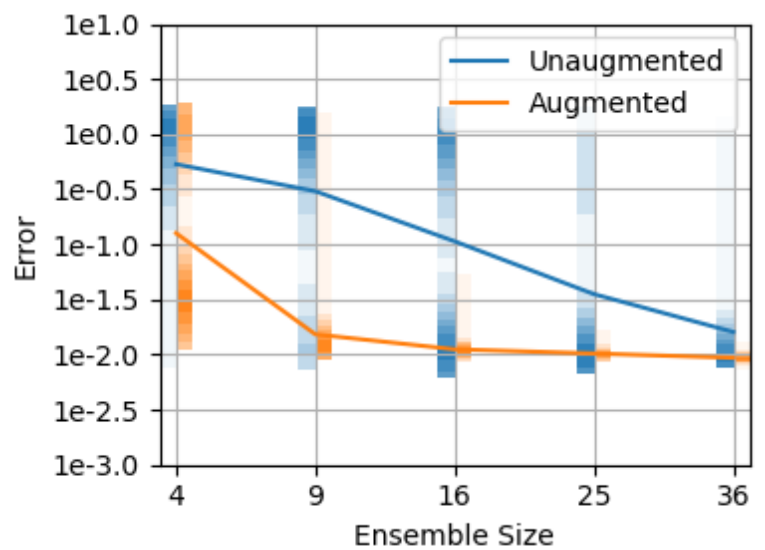

(e) KS Error

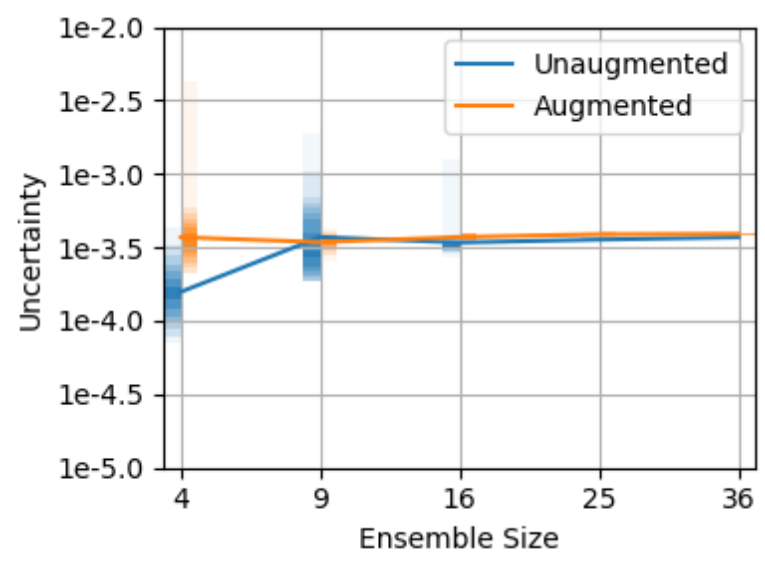

(b) L96 Uncertainty

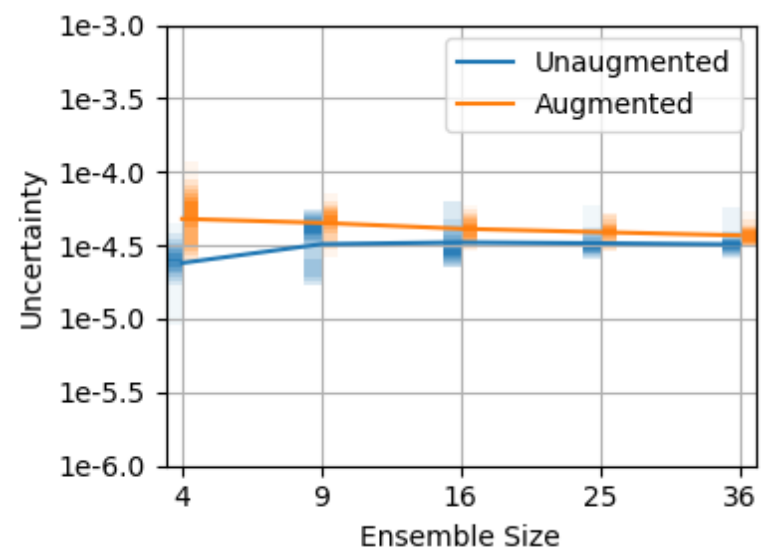

(d) L04 Uncertainty

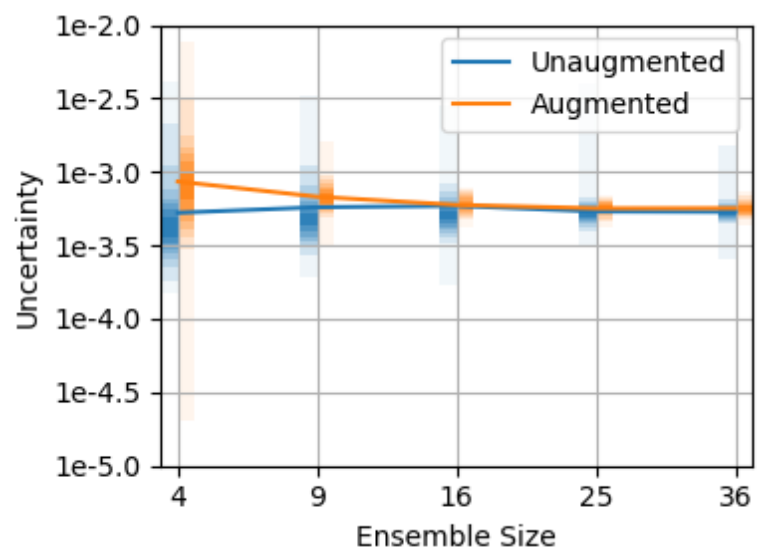

(f) KS Uncertainty

Figure 4.8: Distributions of error and uncertainty for the ETKF with augmentation applied to model problems in the reproduction scenario 
computational cost significantly.

For all three model problems, augmentation works well for all ensemble sizes. The error is low from the smallest ensemble size and rapidly approaches its asymptotic limit. The augmented filter provides far more consistent performance across all ensemble sizes, the only exception being the smallest ensemble size of 4 . The error level achieved at ensemble size 9 across all three model problems is similar to that achieved at ensemble size 36, a fourfold reduction in ensemble size and thus computational cost.

For KS, the error distribution is bimodal at the smallest ensemble size of 4 . This is likely due to the limitations of the matching scheme; the small ensemble size leads to greater uncertainty in the choice of best-match background, meaning greater variability between runs. Here it appears that the matching scheme settles on the smaller background covariance as the ensemble size gets larger, leading to a less overestimated covariance at larger ensemble sizes. The uncertainty is slightly overestimated at small ensemble sizes for both KS and L04, though they decrease with larger ensemble size and error performance is not significantly compromised. L96 on the other hand accurately estimates the uncertainty even from the smallest ensemble size of 4; the only change with larger ensemble size is reducing the spread.

All in all, this demonstrates that the ensemble size required to match an appropriate background distribution is significantly smaller than the ensemble size required to resolve the forecast covariance. This key insight is what enables augmented runs to maintain the accuracy of well-resolved unaugmented runs at reduced computational cost.

\section{Number of Backgrounds}

Using L96, we can examine the effects of different values of $n_{B}$ on the performance at large and small ensemble sizes. There are three scenarios considered here: $n_{B}=10, n_{B}=50$, and $n_{B}=764$. These correspond to heavy clustering for each background, moderate clustering, and no clustering at all (i.e., each forecast ensemble produced by the generating run is its own background, leading to 764 backgrounds). The results for $n_{B}=50$ were already shown in Figure 4.8. The results for $n_{B}=10$ and $n_{B}=764$ are shown in Figure 4.9.

The distance-based classification is sufficiently powerful to choose among large numbers of unclustered backgrounds, as is evidenced by the nearly ideal performance for $n_{B}=764$. Furthermore, the results show little difference between $n_{B}=50$ and $n_{B}=764$, suggesting that the level of clustering in $n_{B}=50$ is appropriate for this particular system. There is a slight tradeoff in performance between small and large ensemble sizes; at $n_{B}=50$, the error mean at ensemble size 4 is lower and the spread is narrower, however it decreases with larger ensemble size more gradually than at $n_{B}=764$. This is because a heavily clustered set of backgrounds is more robust to the poor matching that is likely to occur at very small 


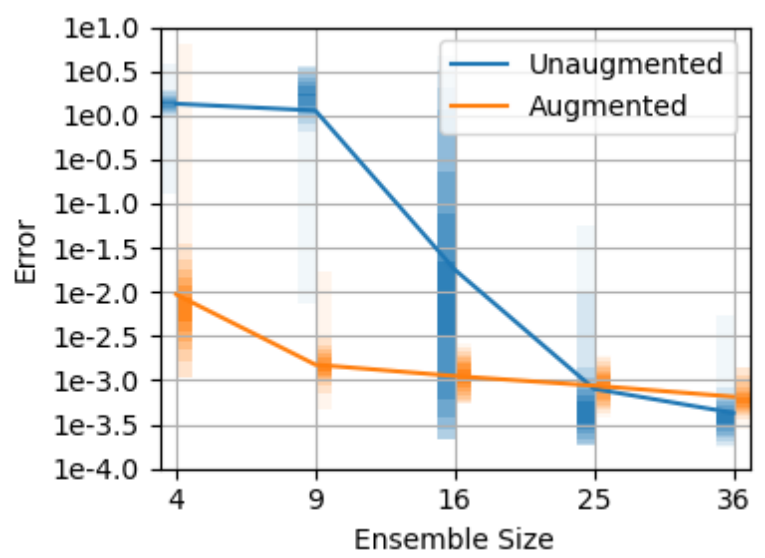

(a) $n_{B}=10$ Error

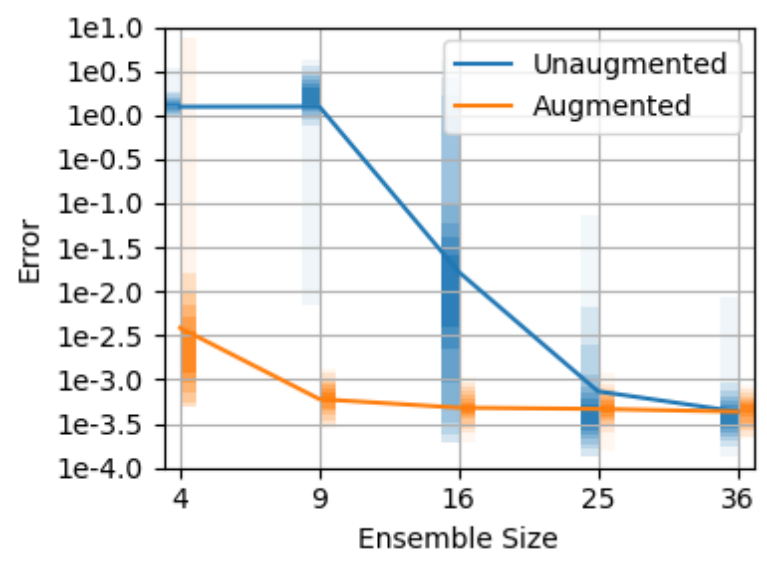

(c) $n_{B}=764$ Error

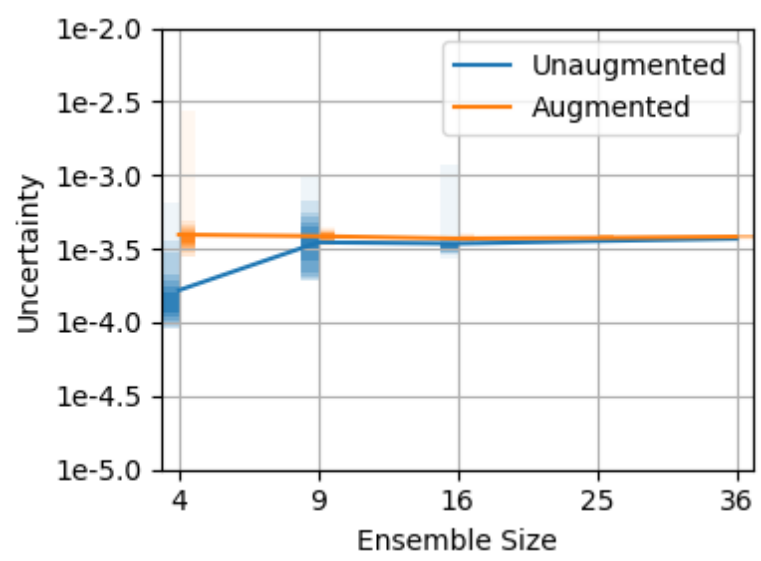

(b) $n_{B}=10$ Uncertainty

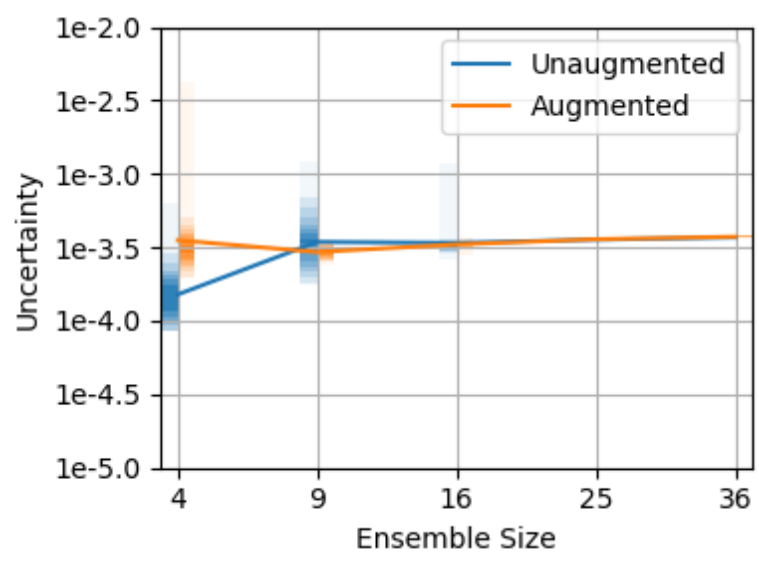

(d) $n_{B}=764$ Uncertainty

Figure 4.9: Distributions of error and uncertainty for the ETKF with augmentation applied to model problems in the reproduction scenario for different $n_{B}$

ensemble sizes, when the natural ensemble is most under-resolved. At larger ensemble sizes, the natural ensemble is resolved enough to reliably match with an appropriate background, so it is advantageous to use a large set of many highly specialized backgrounds. For practical use, the optimal choice of $n_{B}$ therefore is a function of the difference between the ensemble size and the well-resolved ensemble size. The case where $n_{B}=10$ shows the consequences of over-clustering. Even at small ensemble sizes, the robustness to poor matching is more than compensated for by the fact that each background is too much of a "compromise" between different statistical regimes. Consequently, performance suffers across all ensemble sizes, though it is still a significant improvement over the unaugmented filter. 


\section{Bayesian Classification}

We use L96 to illustrate the performance of Bayesian classification as described in Section 3.3.2 compared to the more straightforward distance-based matching from Section 3.3.1. We consider the same three levels of clustering again: $n_{B}=10, n_{B}=50$, and $n_{B}=764$. The results are shown in Figure 4.10.

These results highlight how distance-based and Bayesian classification answer slightly different questions. Bayesian classification assumes that the forecast ensemble is drawn from one of the background distributions and attempts to determine which distribution is most likely. In the case where $n_{B}=764$ (Figures 4.10e and 4.10f), the assumption that each forecast ensemble could have been produced by a background distribution holds true, and the performance of Bayesian classification is nearly ideal. When there is significant background clustering however, none of the distributions are likely matches. In this case, Bayesian classification tends to err in favour of larger covariance ensembles, as the range of plausible Wasserstein distances is much larger. This is evident in Figures 4.10a to 4.10d, where we see that uncertainty is consistently overestimated, leading to compromised performance. Therefore in practical scenarios where we cannot assume that any backgrounds directly match the forecast ensemble, distance-based classification is a more robust choice.

\section{Number of Artificial Members}

The final decision we make in how to perform augmentation is in the quantity of artificial members generated in an augmented run. If this number is too small, then the augmented ensemble will still be insufficient to resolve the forecast covariance. If this number is too large, then the natural ensemble members are diluted by the artificial ones, and the augmented ensemble resolves the background covariance. If the background covariance is not a nearperfect match for the natural ensemble, then over-resolving the background like this may compromise the performance of the filter. There is therefore an optimal middle-ground where the artificial members make a difference, but still allow the natural members to inform the covariance estimate. In this work, we select the number of artificial members such that the augmented ensemble reaches the well-resolved ensemble size. For example, if we perform an augmented run with L96 using a natural ensemble size of 16, then we generate 48 artificial members to bring the augmented ensemble size to 64 . This is the smallest quantity of artificial members necessary to "fully" resolve the forecast covariance. 


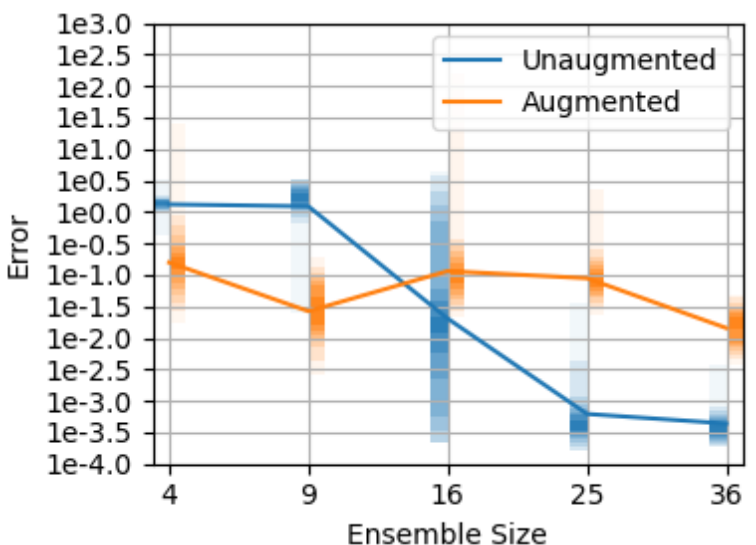

(a) $n_{B}=10$ Error

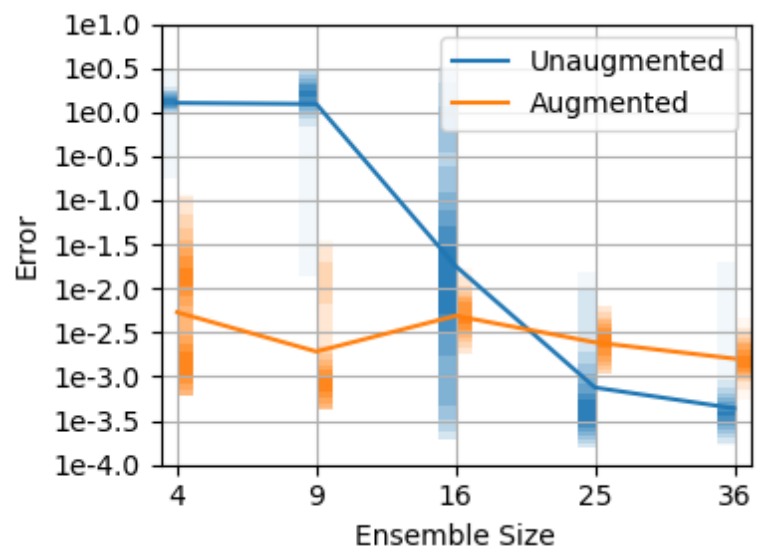

(c) $n_{B}=50$ Error

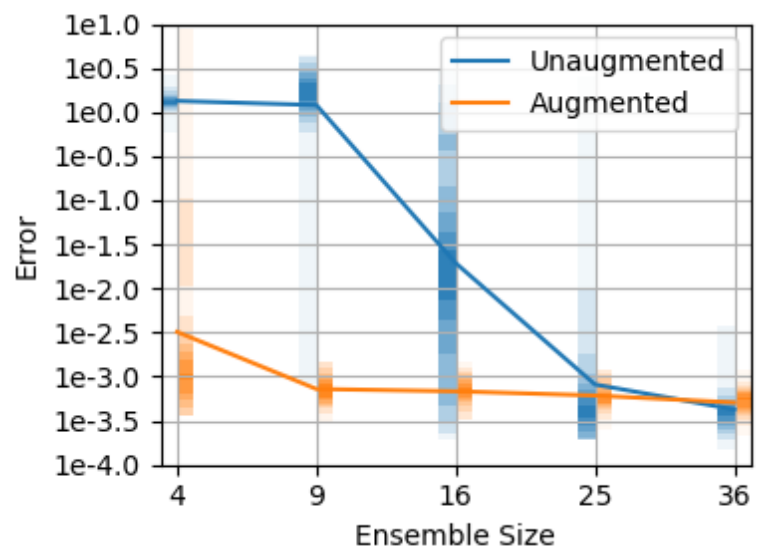

(e) $n_{B}=764$ Error

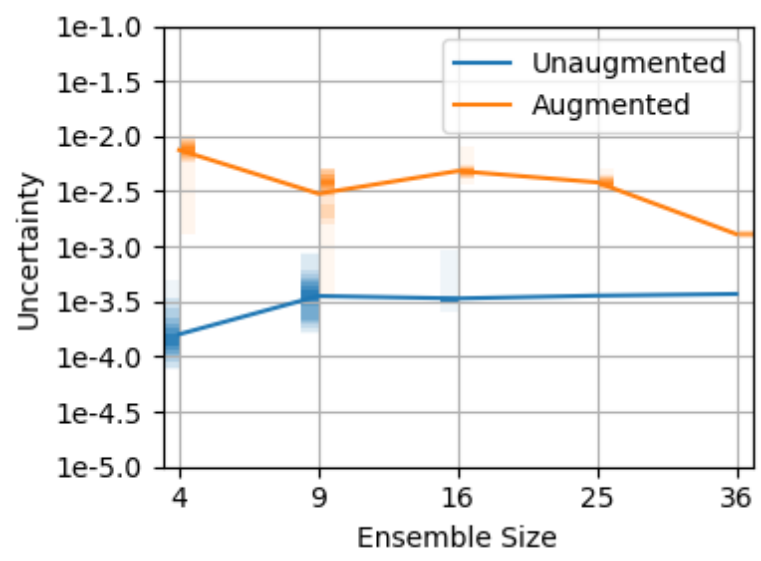

(b) $n_{B}=10$ Uncertainty

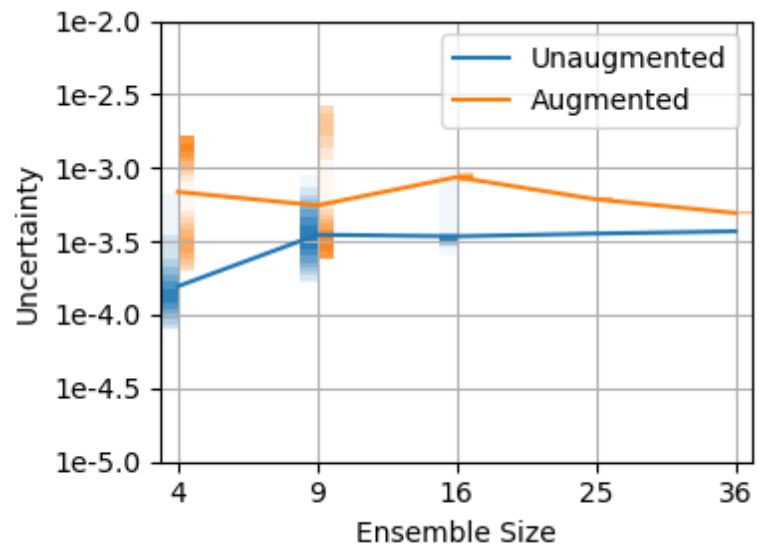

(d) $n_{B}=50$ Uncertainty

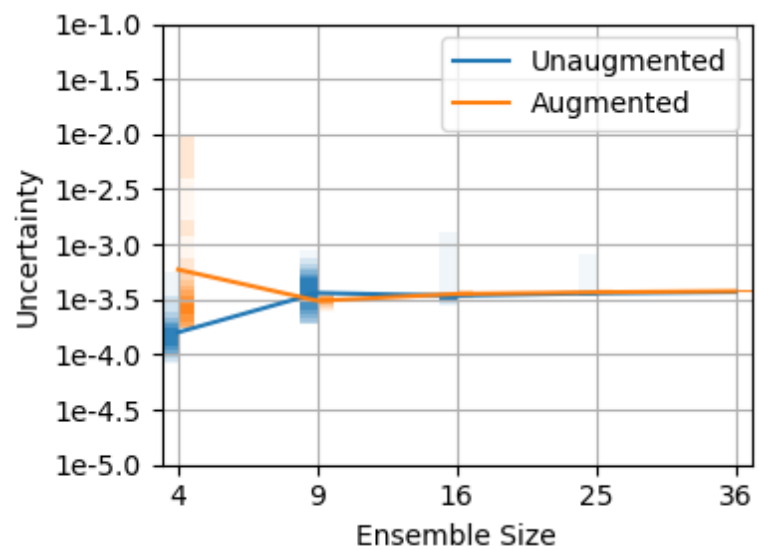

(f) $n_{B}=764$ Uncertainty

Figure 4.10: Distributions of error and uncertainty for the ETKF with augmentation applied to model problems in the reproduction scenario for different $n_{B}$ with Bayesian classification 


\subsubsection{Different Trajectory}

A more practical test of augmentation is where the training data and the test data correspond to different model trajectories. The process model remains the same, but the generating run and the augmented run have different initial conditions. By observing the improvement obtained from backgrounds generated using a different trajectory, this test examines the level of steady statistical information present in the backgrounds. The results are shown in Figure 4.11.

We expect to see improvement if at least one of two conditions holds. The first is if, out of all statistical regimes that may be encountered, the generating runs are reasonably comprehensive. In that case, any possible natural forecast ensemble has an appropriate corresponding background. The second condition is whether there is always steady, persistent statistical information in the backgrounds that may still be relevant to most statistical regimes. While a given state may have a true covariance with dynamic elements not present in the backgrounds, the overall covariance estimate may still be improved by steady-state characteristics obtained from the backgrounds.

This particular test uses a background set of size $n_{B}=50$ generated from the respective model time domains specified in Section 4.1. The augmented runs then start with an offset of half a simulation period from where the generating runs start. They proceed for the same length of time. For example, with L96 the time domain is $[0,2]$ for the generating run and $[1,3]$ for the augmented run.

A consequence of this approach is that different flow behaviour is captured. Most notably, we can see in Figures 4.1, 4.2, and 4.3 that a significant portion of the simulation period is spent in a transient period, where the state develops from initial conditions to its statistically steady behaviour. In this different trajectory scenario, the generating run and consequently the background set include this initial transient, while the augmented run does not. Many backgrounds in the set are therefore superfluous to this augmented run, and we rely on the backgrounds produced from the developed, statistically-steady behaviour. Note that this different statistical behaviour means that the "unaugmented" performance in Figure 4.11 differs from the original baseline results in Figure 4.4.

The exception to this is L04, where the model exhibits transient behavior over the entire time period. The fact that we take full-state observations likely make this case easier than it would otherwise be, because cross-correlation terms are less relevant to the analysis step than the diagonal terms in the covariance. It is however more difficult than the original time domain, as error in the unaugmented filter is higher at all ensemble sizes than in the baseline results. Error in the augmented filter as shown in Figure 4.11c consequently decreases relatively quickly, achieving performance at ensemble size 9 that the unaugmented 


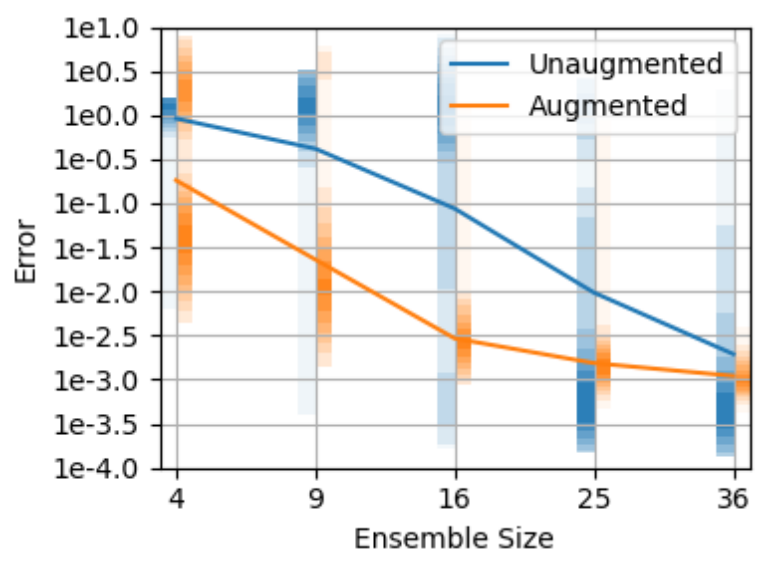

(a) L96 Error

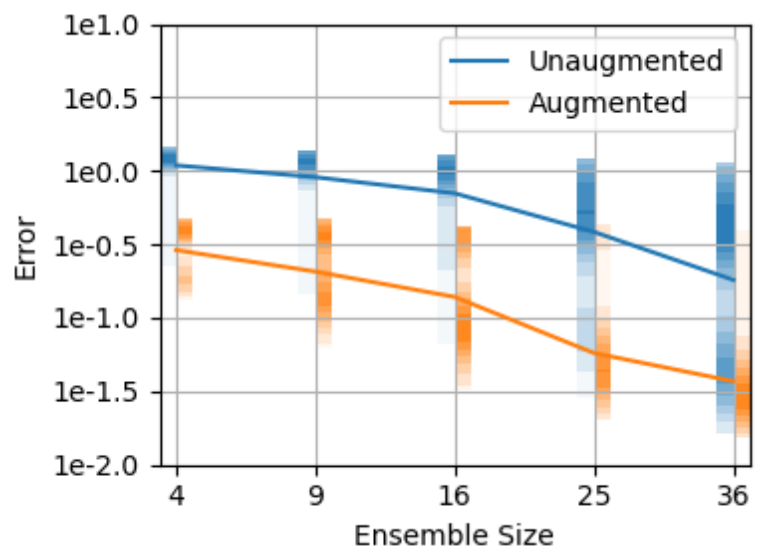

(c) L04 Error

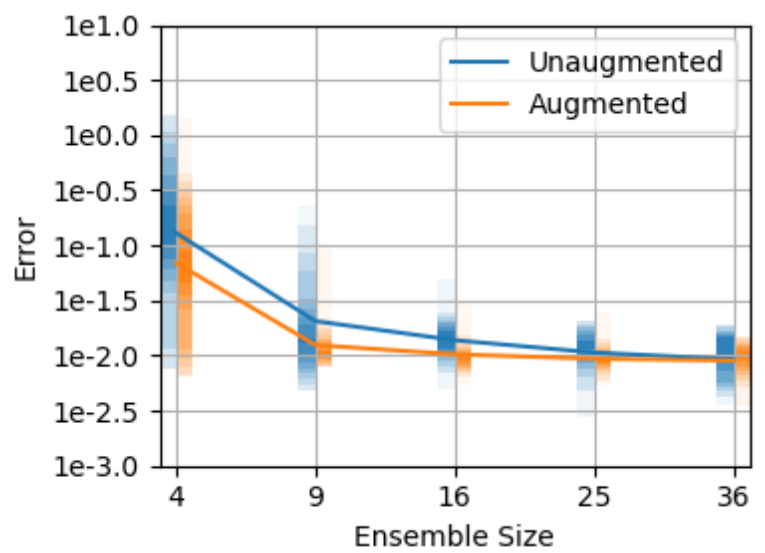

(e) KS Error

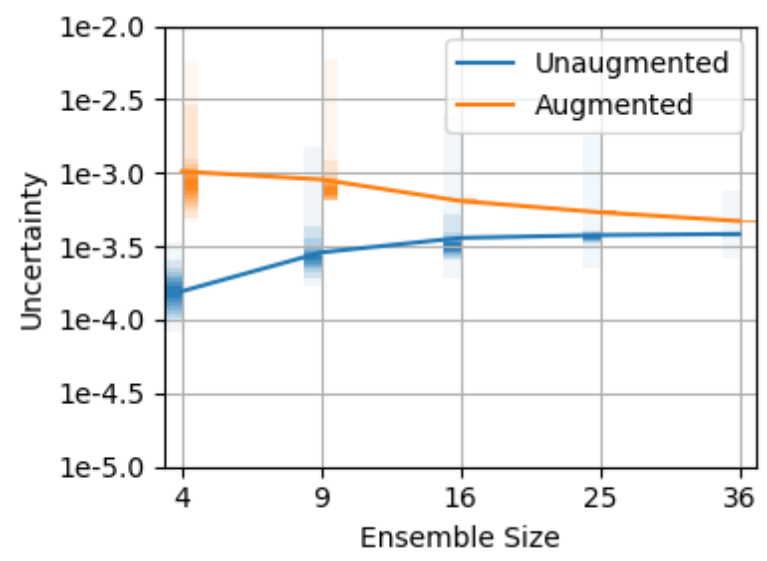

(b) L96 Uncertainty

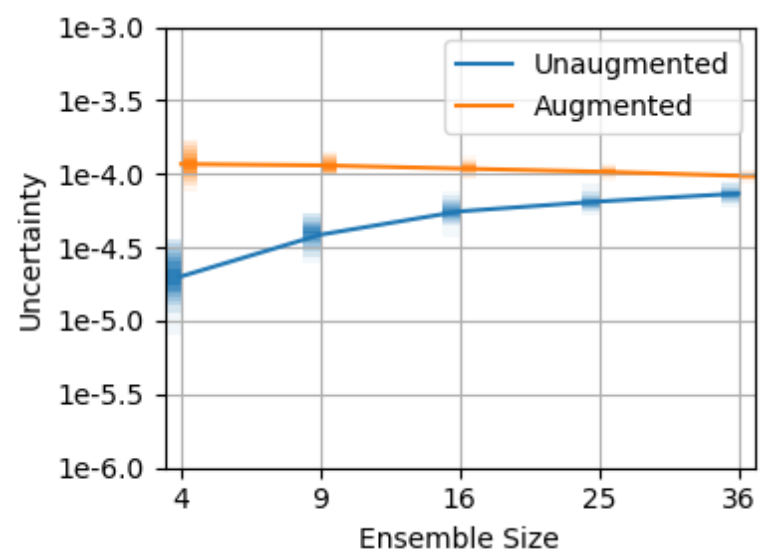

(d) L04 Uncertainty

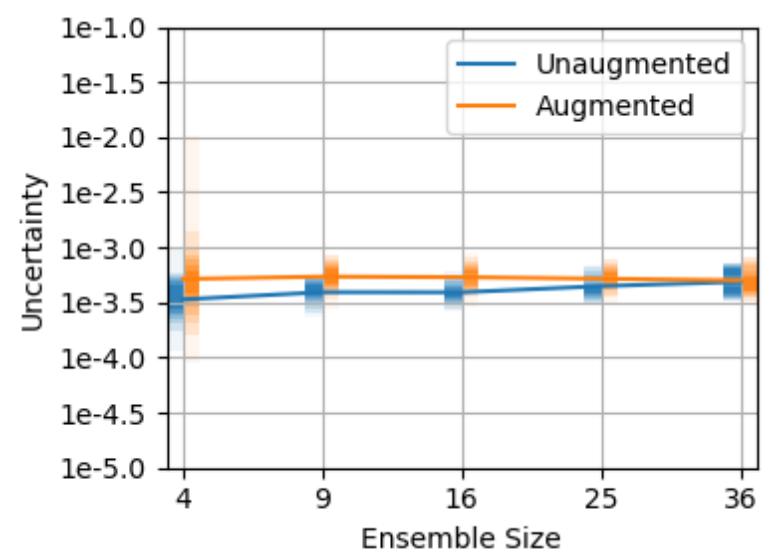

(f) KS Uncertainty

Figure 4.11: Distributions of error and uncertainty for the ETKF with augmentation applied to model problems in the different trajectory scenario 
filter does not achieve until ensemble size 36. The uncertainty plot in Figure 4.11d suggests that the background set contains covariances that are appropriate for this scenario, however other nuances in the background distributions prevent the error from reaching its minimum within the range of ensemble sizes shown.

For L96, there is significant difference between the backgrounds collected from $t \in[0,2]$ and necessary for $t \in[1,3]$. Error in Figure 4.11a is highly bimodal at the smallest ensemble size, but this diminishes quickly as the ensemble size increases. The error spread remains wide for the unaugmented filter across all ensemble sizes, whereas the augmented filter is much more consistent, even where the mean performance is comparable. The clearest example is at ensemble size 16 with the augmented filter and 36 with the unaugmented filter; the augmented filter's error stays within an order of magnitude and the unaugmented filter's error spreads across almost three orders of magnitude. As with L04, the uncertainty plot in Figure $4.11 \mathrm{~b}$ suggests that the background covariances are overestimated for this trajectory.

For KS, the unaugmented performance over $t \in[30,90]$ is significantly better than over $t \in[0,60]$. Augmentation however still demonstrates improvement. Though the mean error traces in Figure 4.11e are similar, the error spread in the unaugmented filter is much wider than in the augmented one. The augmented filter converges tightly on the asymptotic error limit by ensemble size 9, while the unaugmented filter does not until ensemble size 25-36, representing a $3-4 \times$ reduction in ensemble size. In Figure 4.11f, we see that the unaugmented filter underestimates uncertainty at most ensemble sizes, whereas the augmented filter again converges quickly to the asymptotic limit. We can therefore say that the background set generated for KS captures the relevant statistical regimes much better (i.e., is more generalizable) than in L96 or L04.

\subsubsection{Parameter Variation}

We are also interested in the performance of augmented runs when the model parameters differ between the training data and test data. For L96 and L04, the parameter most readily modified is the forcing term $F$, which drives the chaotic motion. That is, a larger value of $F$ will increase the rate at which the state estimate drifts from the true state if uncorrected, while a smaller value of $F$ will decrease it. Increasing this forcing term allows us to test the performance of augmentation when the process model is more chaotic than was anticipated when generating the backgrounds. The results are shown in Figure 4.12.

With L96, the forcing term is $F=8$ for generating runs and $F=10$ for augmented runs. With L04, the forcing term is $F=15$ for generating runs and $F=18$ for augmented runs. For both L96 and L04, the background set is robust to changes in the forcing term. 


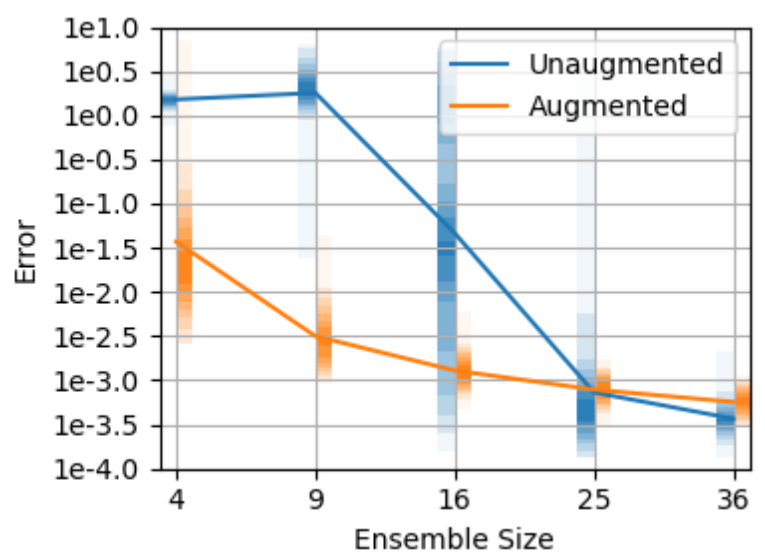

(a) L96 Error

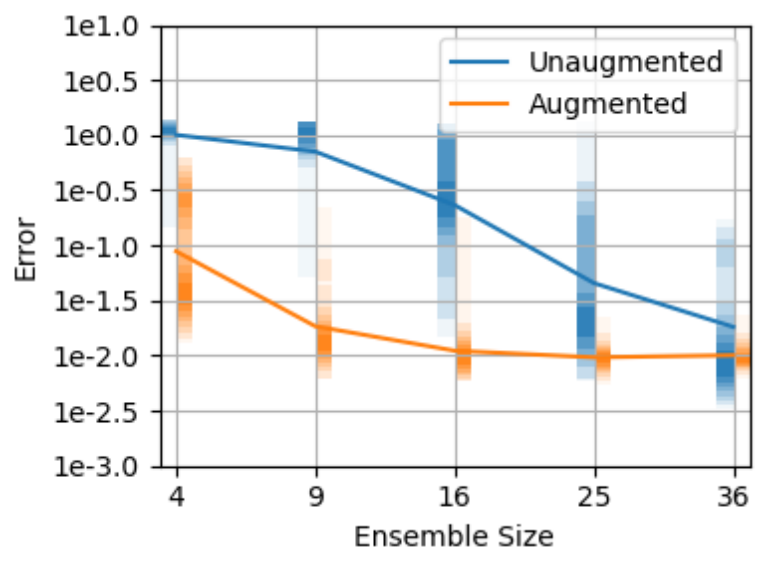

(c) L04 Error

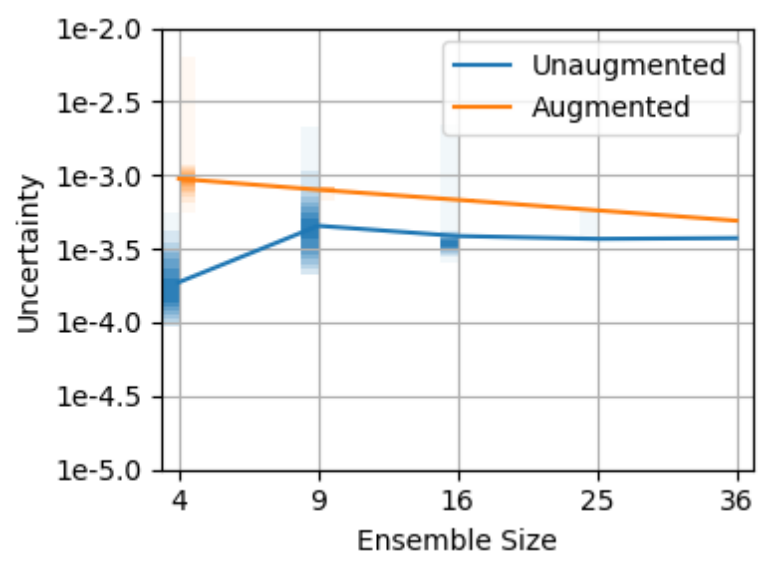

(b) L96 Uncertainty

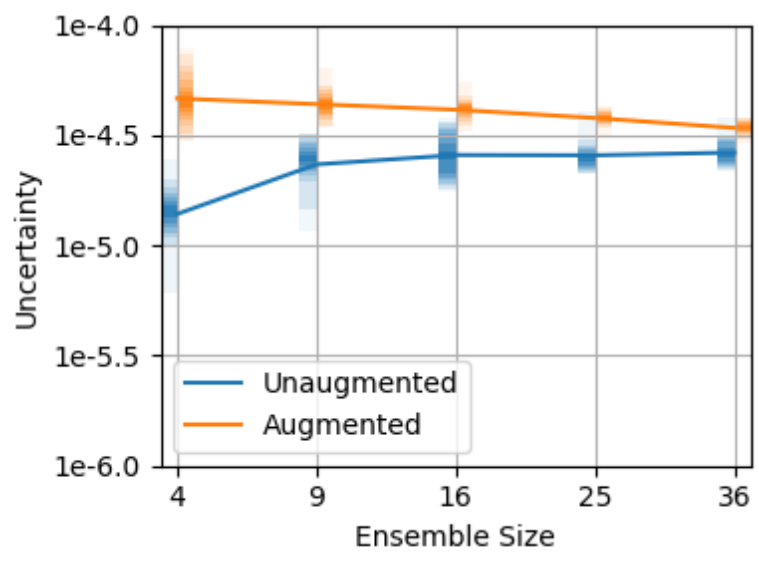

(d) L04 Uncertainty

Figure 4.12: Distributions of error and uncertainty for the ETKF with augmentation applied to model problems in the parameter variation scenario

Except for very small ensemble sizes, the augmented filter is able to consistently converge to the true solution, suggesting that at least for this case, the background set is sufficiently generalizable such that it remains effective for different values of $F$. The augmented filter with L96 achieves stable, converged performance at ensemble size 9 that the unaugmented filter does not achieve until an ensemble size of approximately 20. The augmented filter with L04 achieves an error level at ensemble size 9 that the unaugmented filter does not reach until ensemble size 36 . As with the different trajectory scenario however, the available backgrounds in the set do overestimate the forecast covariance for both L96 and L04. This performance can likely only be improved with generating runs using the new forcing terms. 


\section{Chapter 5}

\section{Reacting Flow Simulation}

Now that we have demonstrated the utility of augmentation in principle using the model problems in Chapter 4, we consider physical simulations of a two-dimensional reacting flow. This shows how augmentation might perform in practice using realistic physical systems with many of the same characteristics as the model problems considered. We discuss the interfacing between flow simulation and DA in Appendix B.

We start this chapter by defining two reacting flow scenarios: a statistically stationary developed diffusion flame and an extinction event. We then discuss the assessment procedure for this chapter, which differs from that in Chapter 4. We then present baseline results of applying an ETKF with a large ensemble size and with a deficient ensemble size. We finally consider two scenarios for augmentation: the reproduction case, and the different trajectory case. For the extinction event, we only consider the reproduction case.

\subsection{Problem Definitions}

The reacting flow we consider is a non-premixed propane-oxygen diffusion flame. The simulation is performed with Raptor, a finite-volume solver developed by Oefelein et al. at Sandia National Laboratories [25]. The simulation captures six chemical species: propane $\left(\mathrm{C}_{3} \mathrm{H}_{8}\right)$, oxygen gas $\left(\mathrm{O}_{2}\right)$, carbon dioxide $\left(\mathrm{CO}_{2}\right)$, water $\left(\mathrm{H}_{2} \mathrm{O}\right)$, carbon monoxide $(\mathrm{CO})$, and nitrogen gas $\left(\mathrm{N}_{2}\right)$, following the simplified process

$$
\begin{gathered}
2 \mathrm{C}_{3} \mathrm{H}_{8}+3 \mathrm{O}_{2} \rightarrow 6 \mathrm{CO}+8 \mathrm{H}_{2} \mathrm{O}, \\
2 \mathrm{CO}+\mathrm{O}_{2} \rightarrow 2 \mathrm{CO}_{2},
\end{gathered}
$$

to simulate the combustion chemistry.

All flow variables are non-dimensionalized. The flow has a Reynolds number of 12000 


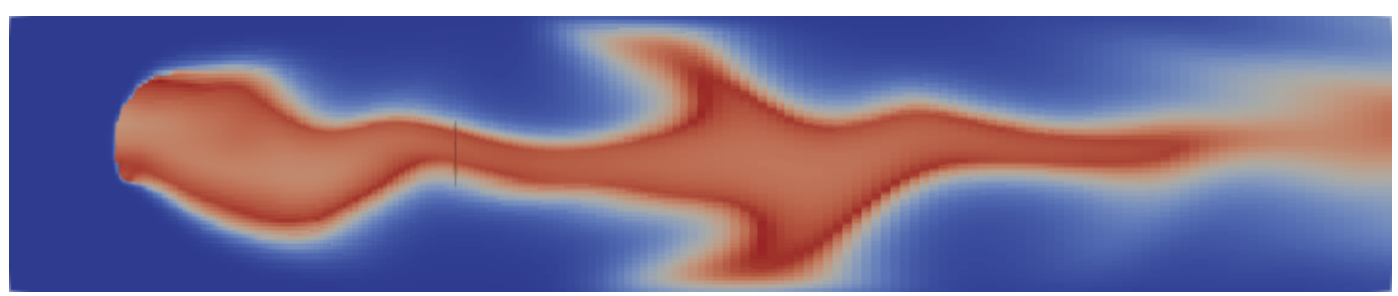

(a) Time $t=0.0$

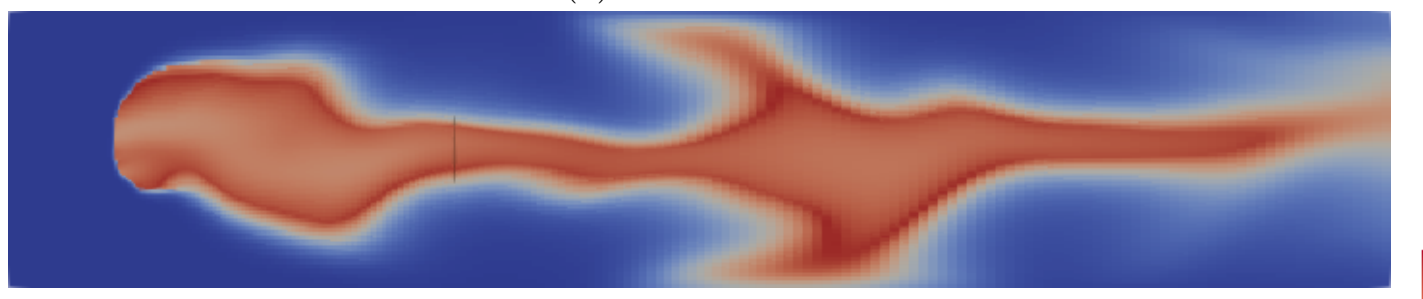

(b) Time $t=0.5$

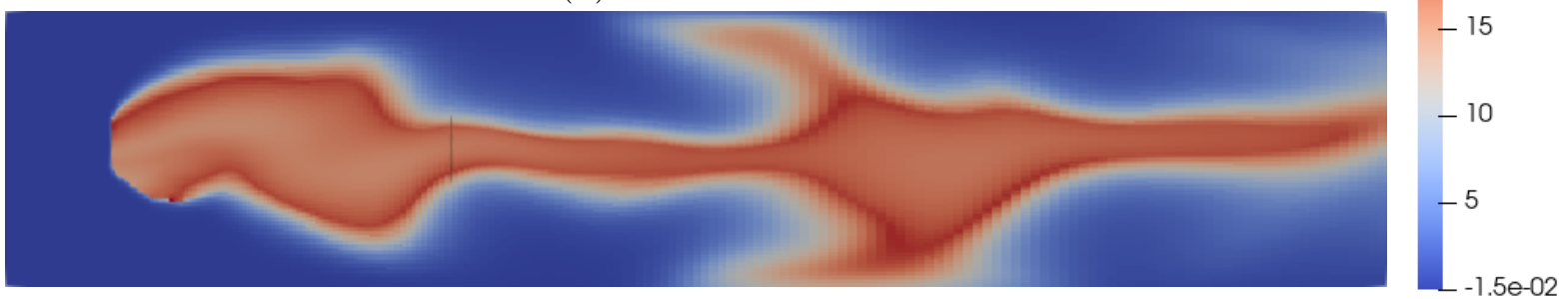

(c) Time $t=1.0$

Figure 5.1: Statistically stationary diffusion flame. The plot is of non-dimensionalized temperature. The direction of flow is from left to right. The gravity vector points to the left.

using the height of the domain as the length scale and the inflow speed as the velocity scale. The inflow boundary on the left side has a uniform flow velocity boundary condition with a narrow propane inlet in the centre. The outflow boundary on the right side has a constant pressure boundary condition. The top and bottom boundaries are slip walls. The simulation is run for a time interval of $[0,0.5]$. The gravity vector points to the left of the domain.

The simulation captures nine flow variables: four fluid dynamic variables (pressure, two velocity components, and temperature) and five explicitly stored chemical mass species fractions (the last, $\mathrm{N}_{2}$, is stored implicitly as 1 - all other species).

\subsubsection{Statistically Stationary Flame}

The first case we consider is that of a statistically stationary, developed flame. Unlike the model problems in Chapter 4, there is no initial transient; we begin this simulation with a fully-developed flow as the initial condition. This is visualized in Figure 5.1, where we see that the flame propagates to the right very slowly. 


\subsubsection{Extinction Event}

The second case we consider is an extinction event resulting from the shutdown of reactant flows. We accomplish this by disabling the inflow boundary on the left side of the spatial domain. The flow then develops as shown in Figure 5.2. Unlike the stationary flame, this flow is entirely transient and moves extremely quickly. We do not expect a fully transient background set to generalize, therefore we only consider the reproduction scenario when performing augmentation.

\subsubsection{Observation Model}

For the observation model, we choose to replicate the effects of a PIV-type 2D velocity measurement and 2D temperature measurement. While 2D temperature measurements are challenging in practice, they represent an important thermodynamic state variable and can potentially be accessed by, for example, Rayleigh scattering [13] or PLIF thermometry [5]. The pressure and chemical species mass fractions are not measured. The velocity measurements are obtained by averaging the computational velocity field over a PIV interrogation window and then adding zero-mean Gaussian noise with standard deviation 0.063. Adjacent PIV interrogation windows have $50 \%$ overlap. The temperature measurements are obtained by sampling the computational temperature field at a point and then adding zero-mean Gaussian noise with standard deviation 0.13. For non-dimensionalized flow variables of order 1 , these correspond to signal-to-noise ratios of roughly 16 and 8 respectively. We choose this limited set of observed flow variables to assess DA's ability to correct unobserved flow variables exclusively through cross-correlation terms in the forecast covariance.

We consider a higher spatial density of temperature observation nodes than PIV interrogation windows. The observation model consists of a uniform $26 \times 6$ mesh of PIV observation windows and a uniform $51 \times 11$ mesh of temperature observation nodes over the domain shown in Figures 5.1 and 5.2. The observation data achieves the spatial resolution shown in Figure 5.3. As the figure shows, both velocity and temperature are spatially under-resolved. By using such a sparse observation model, we assess DA's ability to estimate the small-scale flow structures not captured by the data.

We choose an observation period of $\Delta t=0.05$ in non-dimensionalized time. This provides observation data that is temporally resolved in the temperature field, however fluctuations in the velocity field occur over a much shorter time scale. This is shown in Figure 5.4, where for the statistically stationary flame (as in Figure 5.1), we plot the velocity and temperature over time for a point at the centre of the domain. The figure considers only one observation period, showing that the observation model leaves the velocity field temporally 


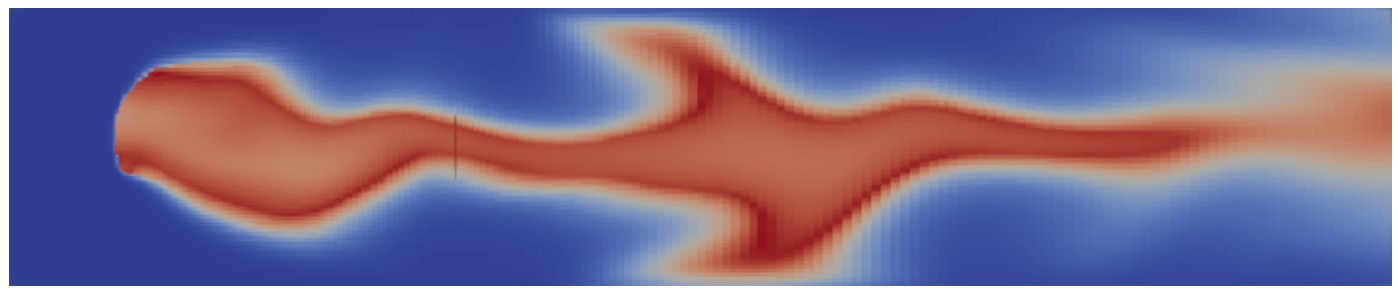

(a) Time $t=0.0$

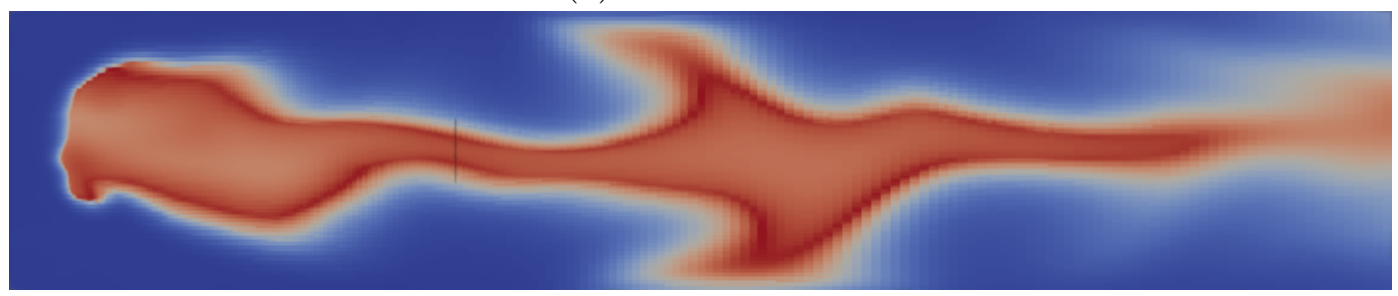

(b) Time $t=0.1$

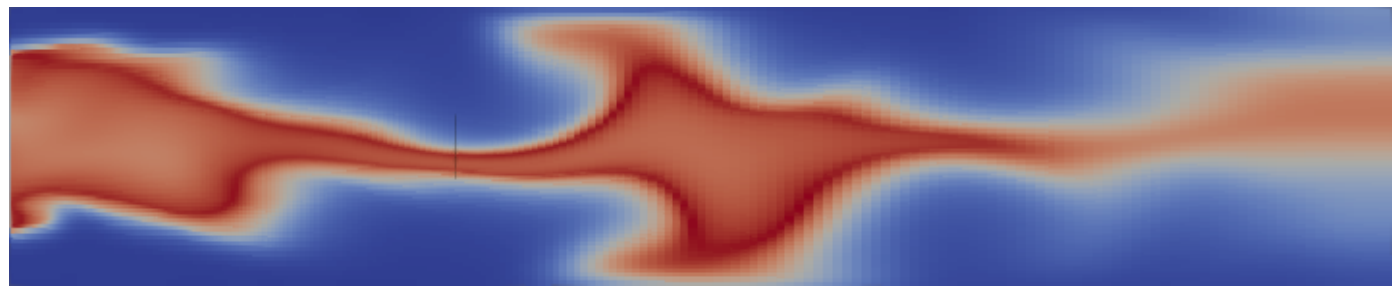

(c) Time $t=0.2$

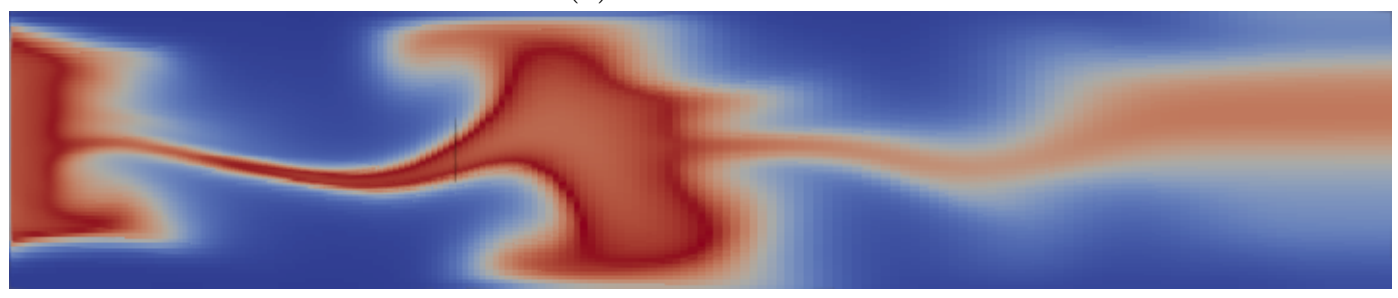

(d) Time $t=0.3$

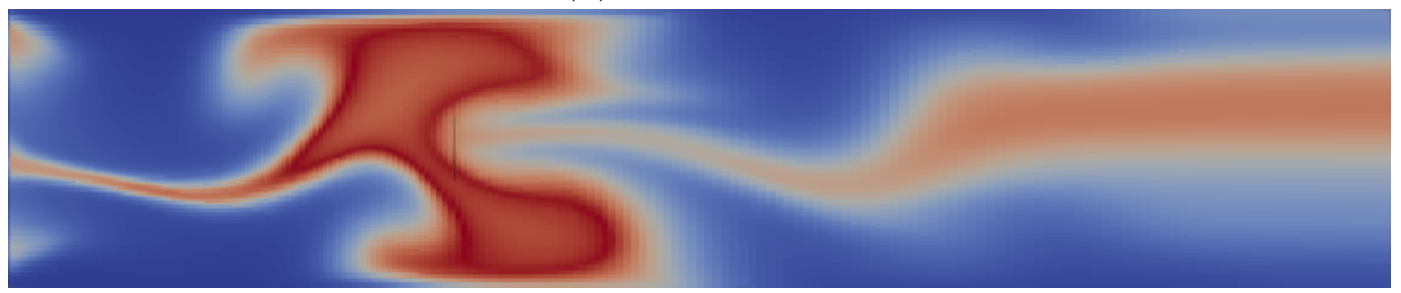

(e) Time $t=0.4$

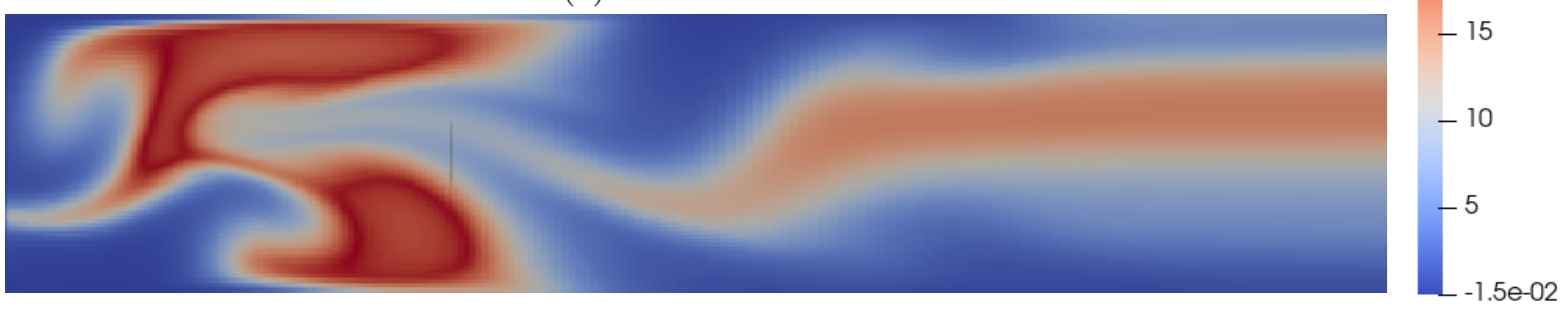

(f) Time $t=0.5$

Figure 5.2: Diffusion flame extinction event. The plot is of non-dimensionalized temperature. The direction of flow is from left to right. The gravity vector points to the left. 


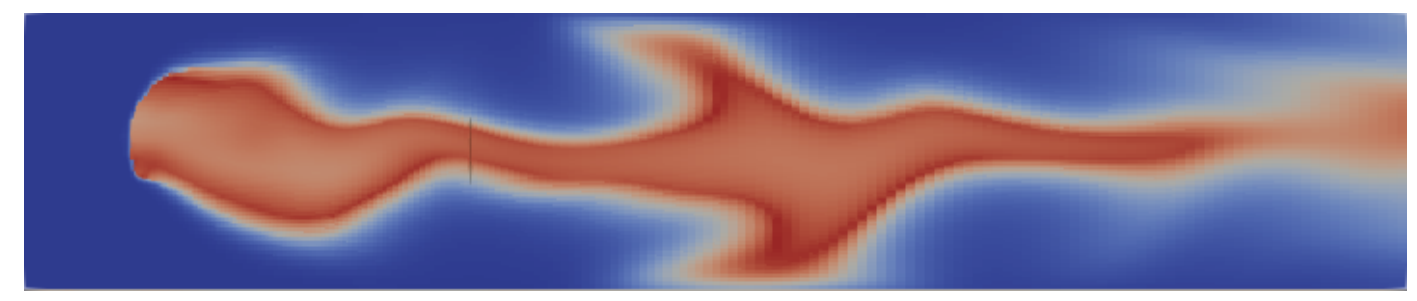

(a) Reference temperature field

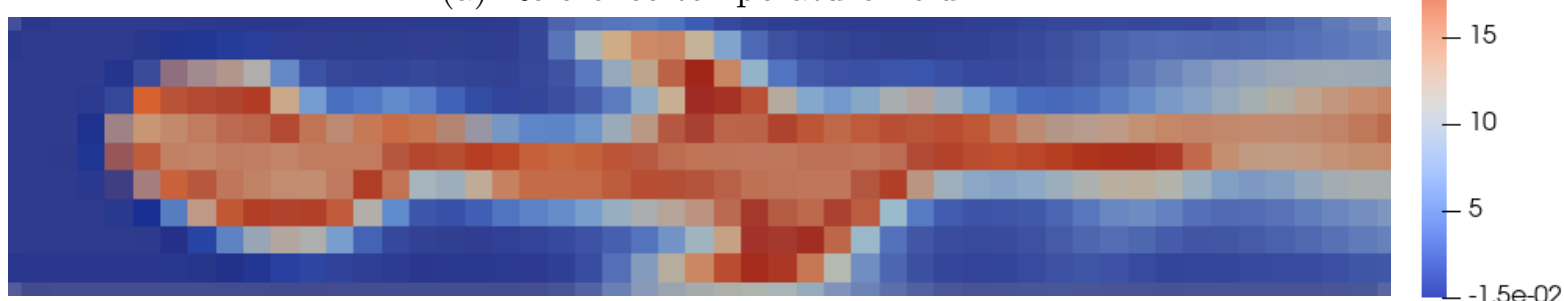

(b) Temperature field observation

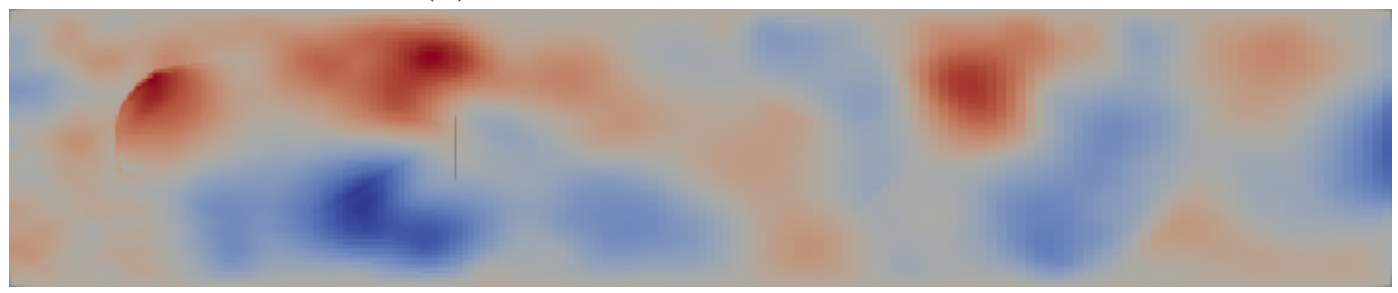

(c) Reference vertical component velocity field

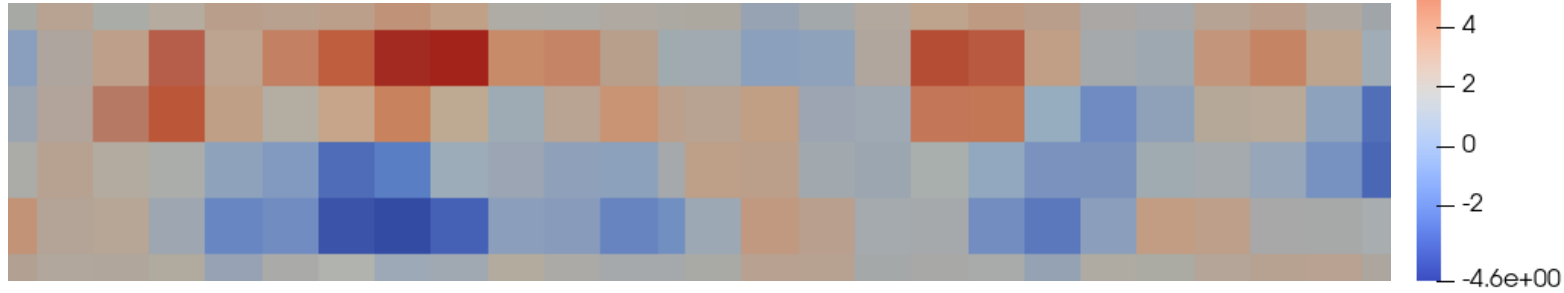

(d) Vertical component velocity field observation

Figure 5.3: Spatial resolution of observation data in velocity and temperature

under-resolved. Much like our choice to use a spatially sparse observation model, this allows us to assess DA's ability to estimate velocity field behaviour not captured by the data.

\subsection{Assessment Procedure}

Because this simulation is far more computationally intensive than the model problems in Chapter 4, the same level of statistical analysis with respect to ensemble size is not feasible. We plot individual runs with the error over time step, broken down by flow variable. Where there is significant variation in the results (i.e., at smaller ensemble sizes), the performance spread is characterized with a sample of five runs. The same initial ensembles used in these five runs are later reused in combination with augmentation. This ensures a fair comparison 


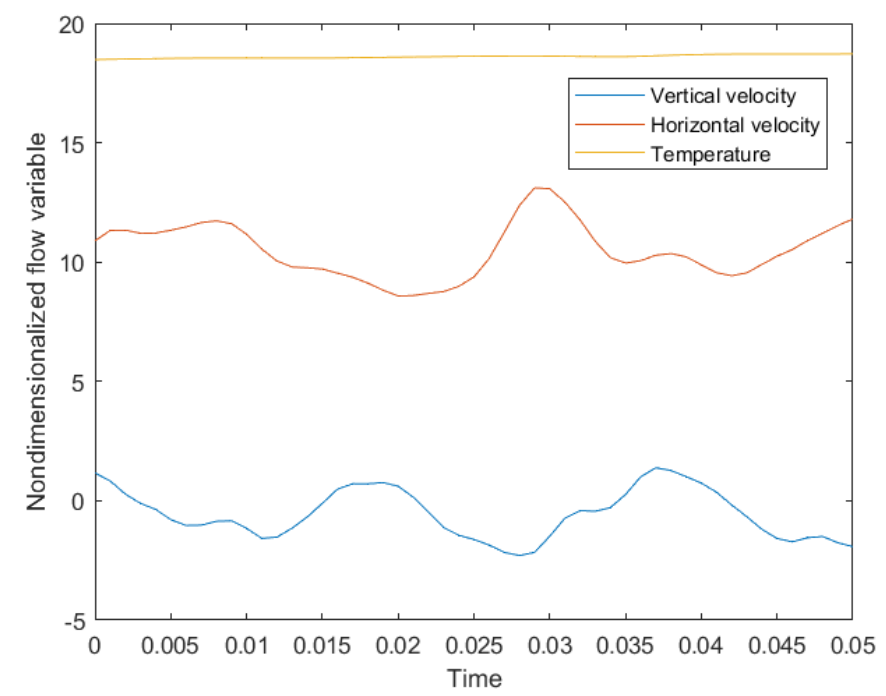

Figure 5.4: Trajectory of nondimensionalized velocity and temperature over one observation period. Measurements taken at centre of domain in a statistically stationary flame case.

between the baseline and augmented results.

To evaluate the performance of the ETKF, we use the same measures of error and uncertainty given in (4.1) and (4.2), respectively, extended to two dimensions and broken down by flow variable. The plots of error and uncertainty over time therefore have nine traces.

There is an issue with the Raptor simulation that affects our ability to resolve the flow with DA. As the stationary flame develops, numerical errors in the pressure and velocity terms tend to accumulate and produce a "checkerboard" effect at the base of the flame, as illustrated in, Figure 5.5. This is a grid-scale and unsteady phenomenon, making it impossible to resolve with our simulated PIV observation model. We therefore measure the error only in the right-hand two-thirds of the spatial domain for the statistically stationary flame. This eliminates the effect of this checkerboard behaviour on the performance of the filter. Because the checkerboard effect does not appear in the extinction event, in that case we measure the error in the state estimate over the whole spatial domain.

\subsection{Baseline Performance}

We define two baseline scenarios that will serve as reference points for evaluating the performance of augmentation. The first is a scenario where the ensemble is sufficiently large to capture the dynamics. This provides the best-case performance and serves as the setup for generating runs. The second is a scenario where the ensemble size is deficient. This provides a baseline example of poor performance that may be compared and contrasted with 


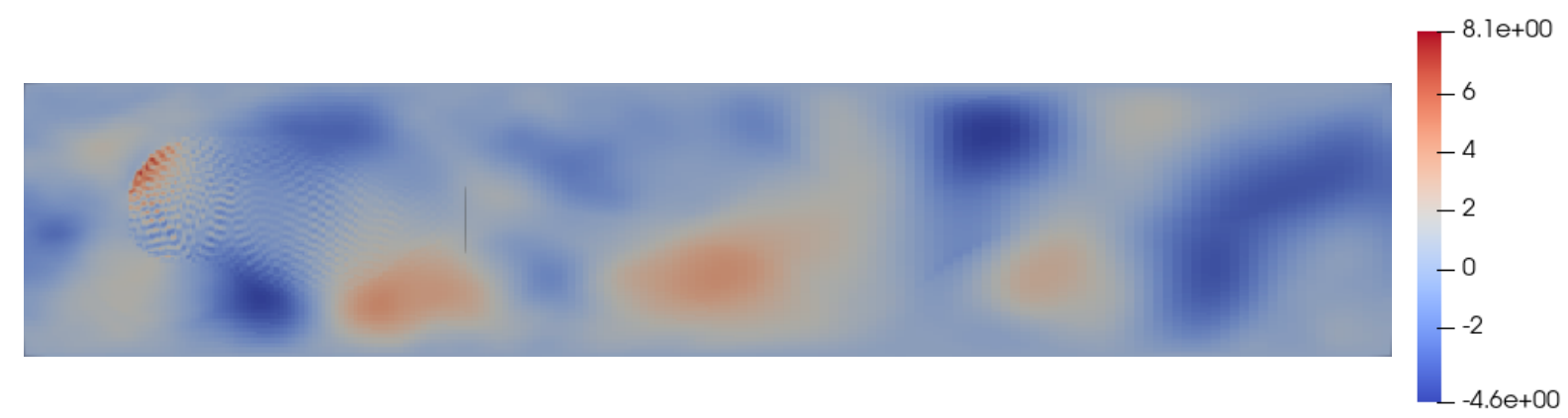

Figure 5.5: Example of "checkerboard" effect in statistically stationary flame at time $t=0.5$. The plot is of non-dimensionalized vertical velocity. The direction of flow is from left to right. The gravity vector points to the left.

augmentation applied to the same ensemble size.

\subsubsection{Large Ensemble Size}

For the well-resolved case with sufficiently large ensemble size, we choose an ensemble size of 48. This is also the generating run for the background set used in augmentation.

For the statistically stationary flow, the ETKF achieves error between $10^{-1.5}$ and $10^{-0.5}$ for the velocity components and pressure (usually around $10^{-1}$ ), and between $10^{-2.5}$ and $10^{-1}$ for species fractions and temperature. Uncertainty is well-correlated with error and converges down to similar values, however occasional analysis updates will cause uncertainty to spike. For nondimensionalized flow variables of order 1, this corresponds to approximately $10-15 \%$ error in the velocity and pressure and $0.3-10 \%$ error in species fractions and temperature. This velocity error is lower than the level of velocity fluctuation shown in Figure 5.4, supporting that the ETKF achieves super-temporal resolution. An example of the performance is given in Figure 5.6.

For the extinction event, the filter achieves error between $10^{-1.5}$ and $10^{-2}$ for velocity and pressure and between $10^{-2}$ and $10^{-2.5}$ for species and temperature. Uncertainty converges to be roughly half an order of magnitude lower, though still well-correlated with error. For nondimensionalized flow variables of order 1, this corresponds to approximately 1-3\% error in the velocity and pressure and $0.3-1 \%$ error in species fractions and temperature. The error and uncertainty are much lower and more consistent across flow variables compared to the statistically stationary flame. We hypothesize that this is because the transient flow in the extinction event correlates very strongly over time and across flow variables, much more so than the statistically stationary flame case. An example of the performance is given in Figure 5.7. 


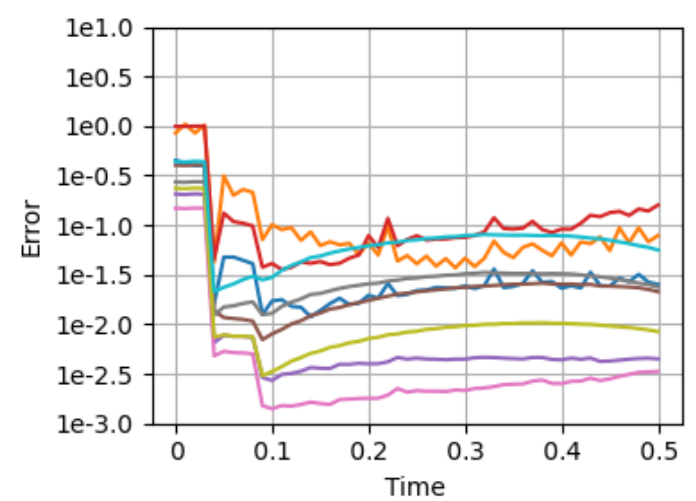

(a) Error

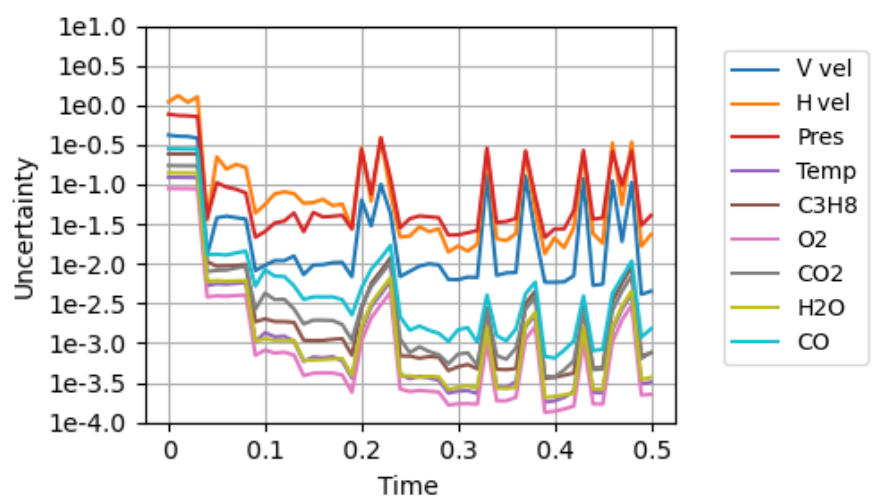

(b) Uncertainty

Figure 5.6: Statistically stationary flame - baseline results with large-ensemble ETKF

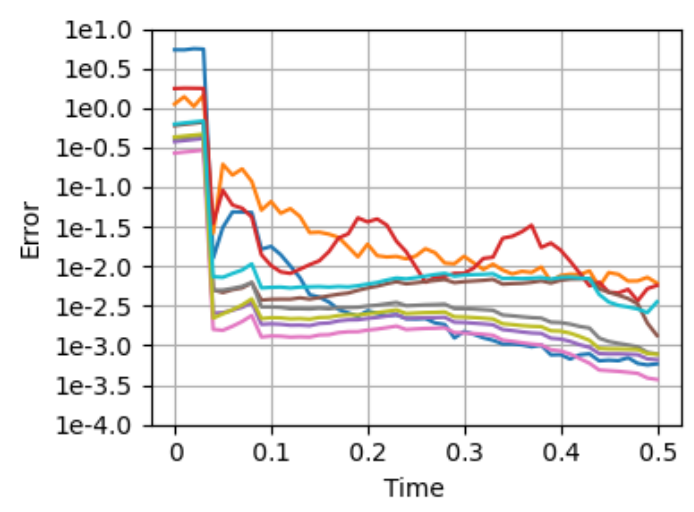

(a) Error

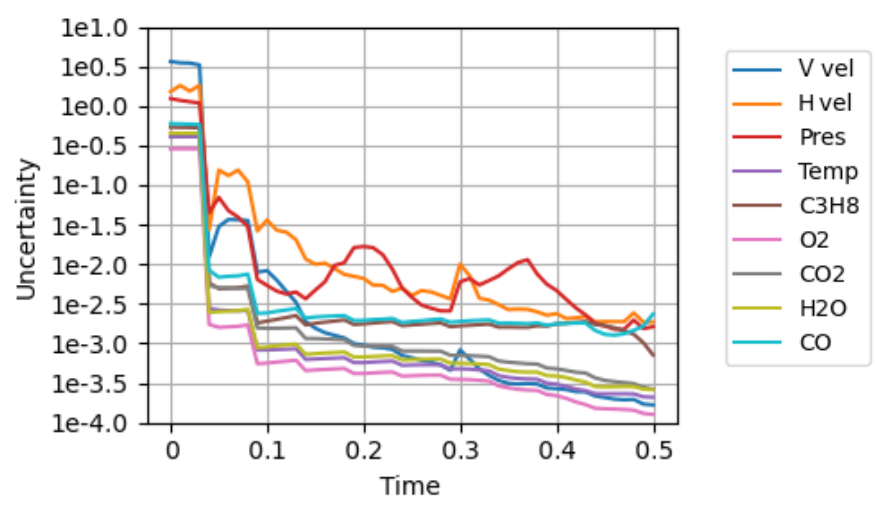

(b) Uncertainty

Figure 5.7: Extinction event - baseline results with large-ensemble ETKF

In both cases, the error decreases quickly after the first observation update, suggesting that the spatial density of observation nodes is more than sufficient to resolve the state of the system. After this first observation, the uncertainty in the ensemble is of similar order to the observation noise and the analysis updates are less significant. The error and uncertainty are strongly correlated over time as well, demonstrating that an ensemble size of 48 is sufficient to capture the uncertainty in the state estimate.

Here we also note the strength of the correlation across flow variables. This causes pressure and species fractions to converge despite not being directly observed. The largest persistent error is in the velocity and pressure components, despite velocity being directly observed via simulated PIV. This is due in part to more rapid fluctuations in the velocity field as discussed in Section 5.1.3, as well as the relatively lower spatial density of PIV windows compared to temperature observation nodes, as was shown in Figure 5.3. 


\subsubsection{Small Ensemble Size}

We choose an ensemble size of 12 , as it yields large error and consistently underestimated uncertainty. The results are shown in Figure 5.8 for the statistically stationary flame and in Figure 5.9 for the extinction event.

To represent the variability in performance, two samples are presented here. For the stationary flow and extinction event respectively, Figures 5.8a and 5.9a show typical cases where the error remains high while uncertainty decreases, indicating that the ensemble has converged around a poor state estimate. Figures $5.8 \mathrm{~b}$ and $5.9 \mathrm{~b}$ show a less common result where the error and uncertainty both decrease to extremely low values, comparable to the results with ensemble size 48 .

For the statistically stationary flame, the spread in ETKF performance shown in Figure 5.8c demonstrates consistently underestimated uncertainty between $10^{-2}$ and $10^{-1.5}$ and large error between $10^{0}$ and $10^{0.5}$. The error is not consistently large however; the occasional sample run may achieve a low error consistent with the large-ensemble case.

For a typical case as shown in Figure 5.8a, the error is virtually unaffected by observation data after each analysis step. The uncertainty decreases with analysis updates just as in Figure 5.6, even approaching a similar order of magnitude. The error however remains within the same order of magnitude as where it started, showing that each analysis step does not make the state estimate any more accurate. The ensemble has converged toward a poor state estimate, where it is resistant to further correction due to an underestimated state covariance.

For an atypical case as in Figure 5.8b, the first two analysis updates reduce the error to a degree consistent with the large-ensemble results in Figure 5.6. This is an unusual case where the small ensemble converges toward an accurate state estimate, however it cannot be considered to have captured the statistical characteristics of the system. The error slowly increases with subsequent updates while uncertainty does not, save for an anomalous spike at the last time step. Before that, the error and uncertainty are nearly an order of magnitude apart. As with the model problems, it is this disparate behaviour between uncertainty and error that characterizes an undersized ensemble.

For the extinction event, the spread in ETKF performance shown in Figure 5.9c demonstrates consistently underestimated uncertainty of approximately $10^{-3}$ and large error just below $10^{-0.5}$. As with the statistically stationary flame, the error is not consistently large. An atypical case may again achieve a low error consistent with the large-ensemble case.

For a typical case as shown in Figure 5.9a, there is drop in error and uncertainty after the first analysis update, similar to that in Figure 5.7. The error however does not decrease nearly as much as uncertainty, which continues to decrease with subsequent updates although 


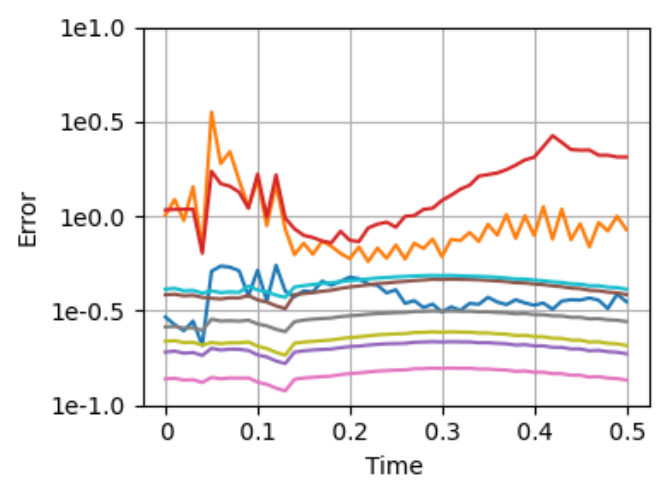

Error

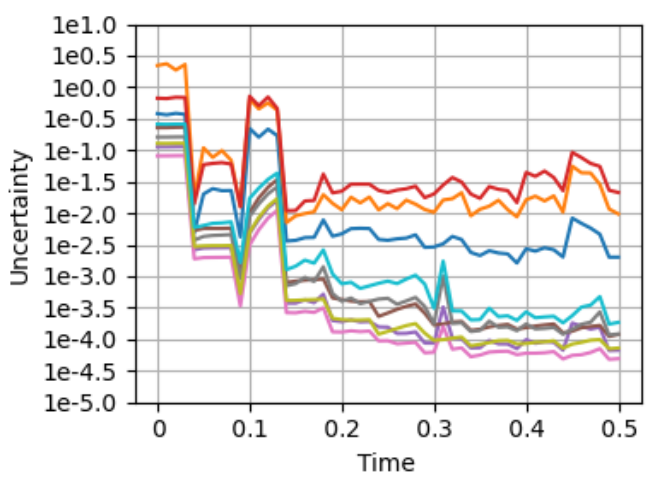

Uncertainty

(a) Typical results with small-ensemble ETKF

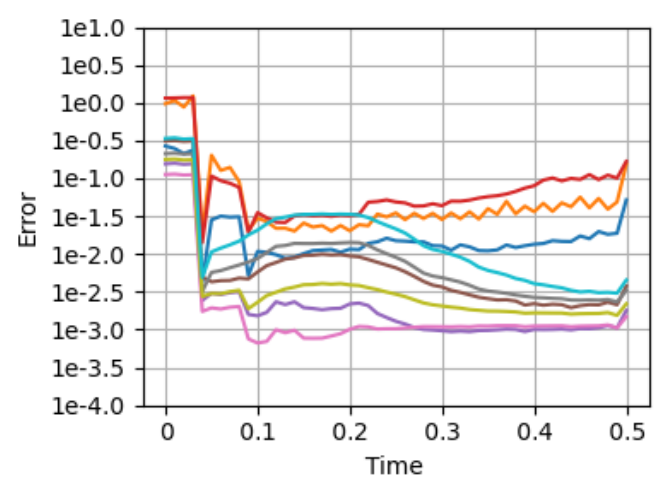

Error

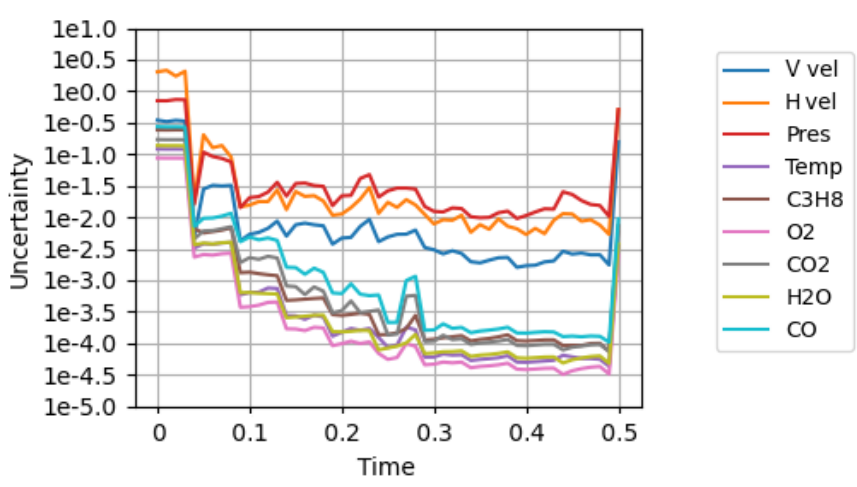

Uncertainty

(b) Atypical results with small-ensemble ETKF

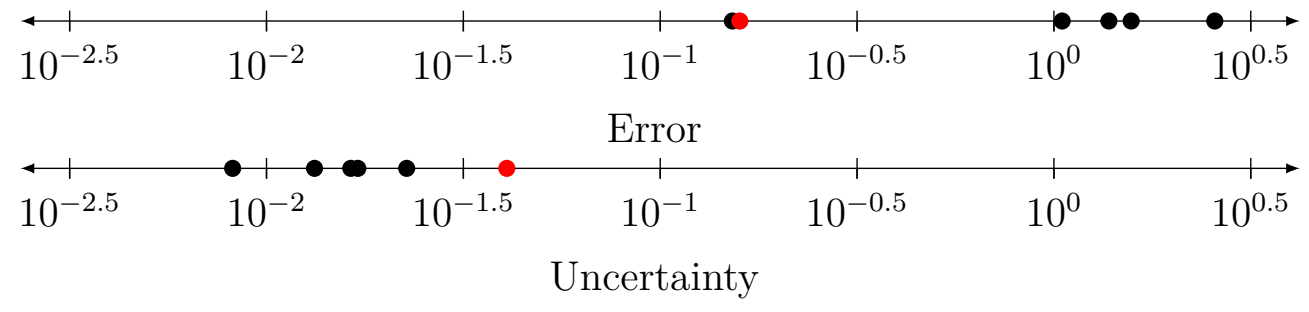

(c) Results spread. Black dots are $n_{\mathrm{en}}=12$ cases. Red dot is $n_{\mathrm{en}}=48$ case.

Figure 5.8: Statistically stationary flame - baseline results with small-ensemble ETKF 


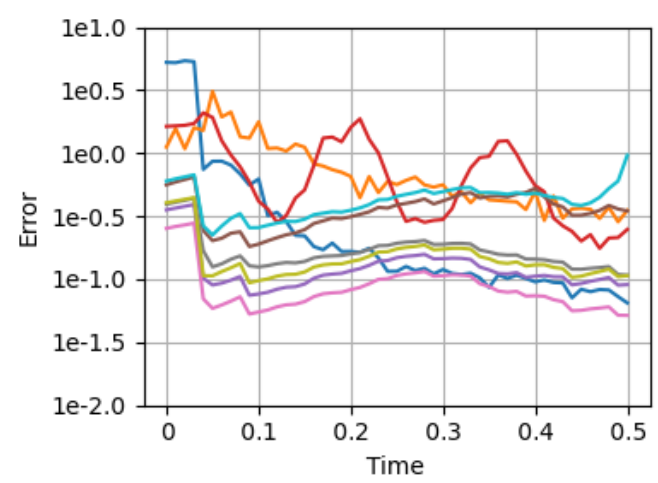

Error

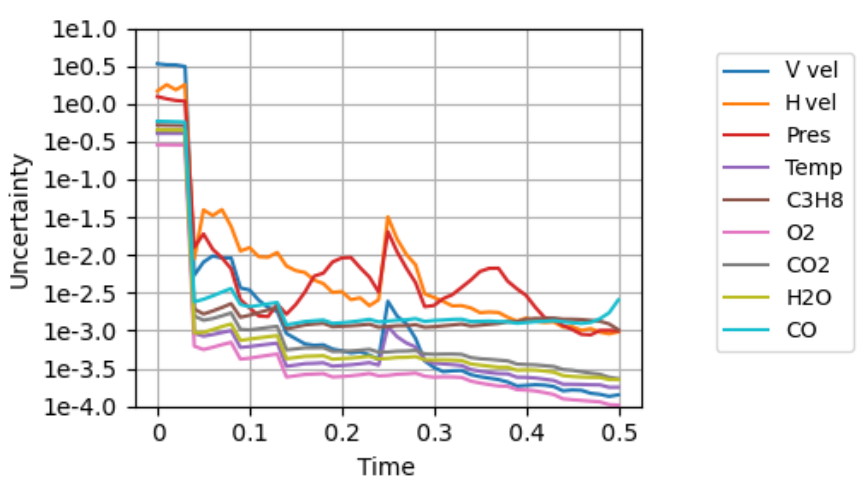

Uncertainty

(a) Typical results with small-ensemble ETKF

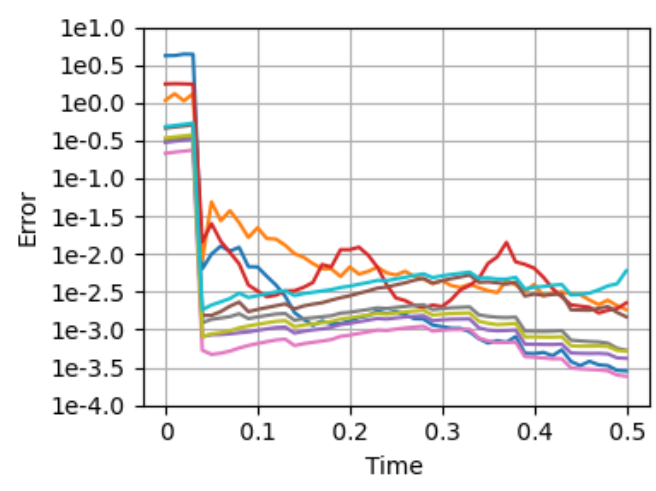

Error

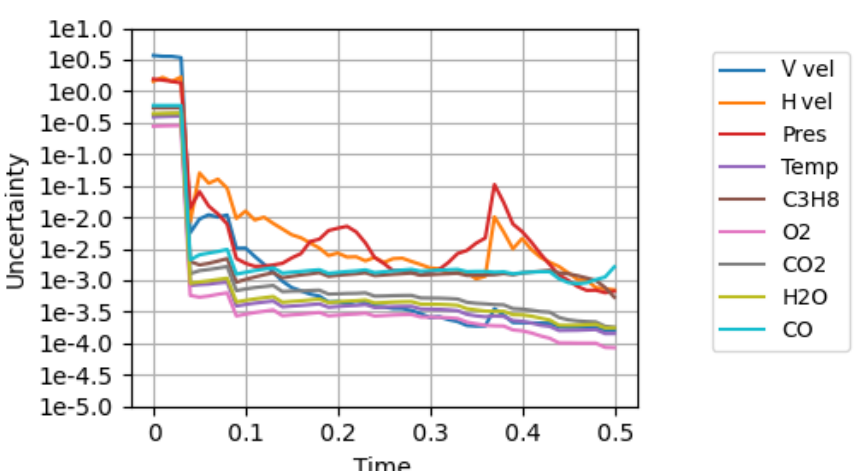

Uncertainty

(b) Atypical results with small-ensemble ETKF

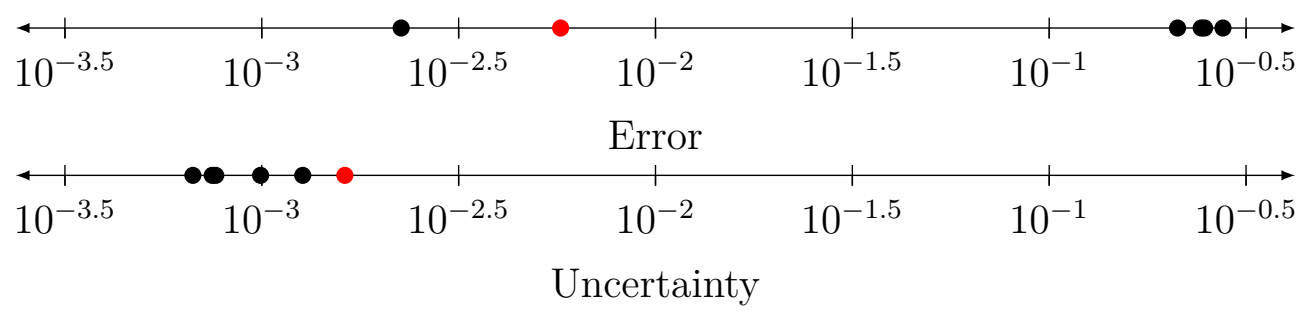

(c) Results spread. Black dots are $n_{\mathrm{en}}=12$ cases. Red dot is $n_{\mathrm{en}}=48$ case.

Figure 5.9: Extinction event - baseline results with small-ensemble ETKF 
error remains high. As with the statistically stationary flame, the ensemble has converged toward a poor state estimate.

For an atypical case as shown in Figure 5.9b, the first observation update immediately brings the error and uncertainty to the order of $10^{-2}$. The final uncertainty is lower than the final error by roughly half an order of magnitude, however this is in line with the largeensemble performance in Figure 5.7. The ensemble convergence after the first update happens to bring the ensemble estimate close to the true state. As shown in Figure 5.9c, this is an unusual level of performance for this ensemble size. It is not certain however that the state estimate would retain its low error indefinitely. It is probable that, like the atypical smallensemble case for the statistically stationary flame, the undersized ensemble would become resistant to correction and drift further away from the true solution.

\subsection{Augmentation}

Two augmentation scenarios are considered here. We start with the reproduction scenario, where the model trajectory in the generating run is the same as that in the augmented run. This is applied to both the statistically stationary flame and the extinction event. We then consider a scenario where these trajectories differ, which tests the applicability of the background set outside of the training data. A background set generated from a fully transient flow is not expected to generalize, so we only consider the different trajectory scenario for the statistically stationary flame. For both the reproduction and the different trajectory scenarios, we produce $n_{B}=50$ backgrounds from one generating run. We use the distance-based classification scheme for both the generating and augmented runs. The large-ensemble case detailed in Section 5.3.1 serves as our generating run. For our augmented run, we use a small ensemble size $n_{\mathrm{en}}=12$ as in Section 5.3.2.

\subsubsection{Reproduction Scenario}

As with the model problems in Chapter 4, we consider the reproduction scenario to illustrate the best-case scenario for augmentation. The results are shown in Figure 5.10 for the statistically stationary flame and in Figure 5.11 for the extinction event.

For the stationary flame, the performance of augmentation is almost ideal. We see from the results spread in Figure 5.10b that the mean error is decreased by an order of magnitude and the mean uncertainty raised by half an order of magnitude, bringing performance almost perfectly in line with the unaugmented large-ensemble case. This is done at an ensemble size of 12 instead of the 48 for the large-ensemble case, and hence a quarter of the computational 


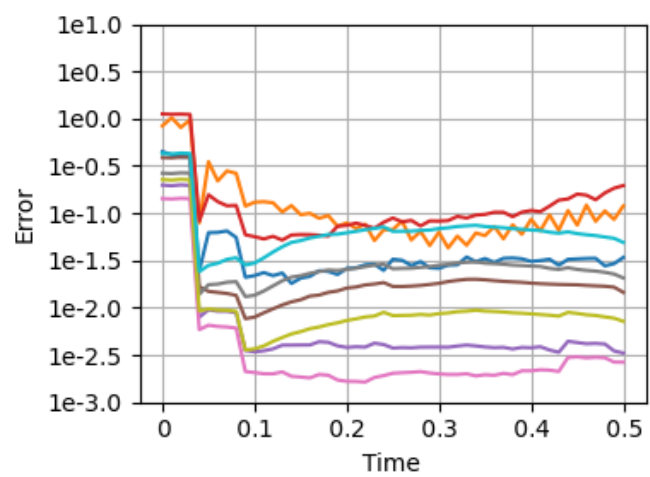

Error

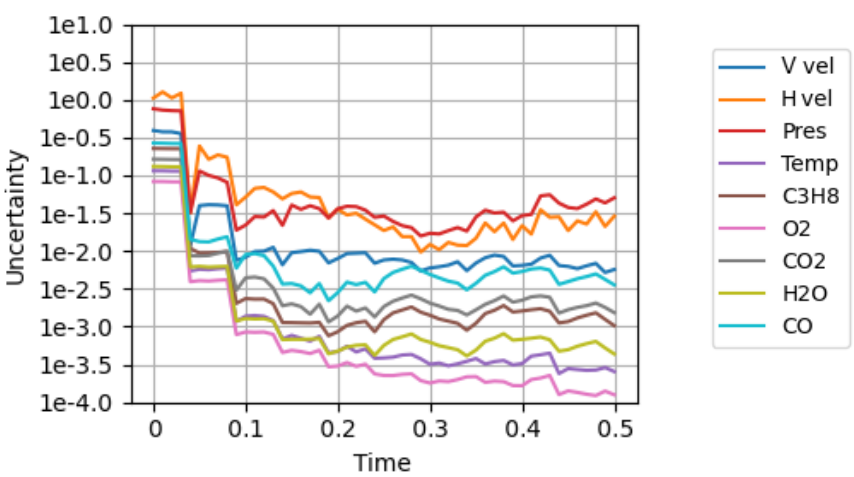

Uncertainty

(a) Example results with small-ensemble ETKF

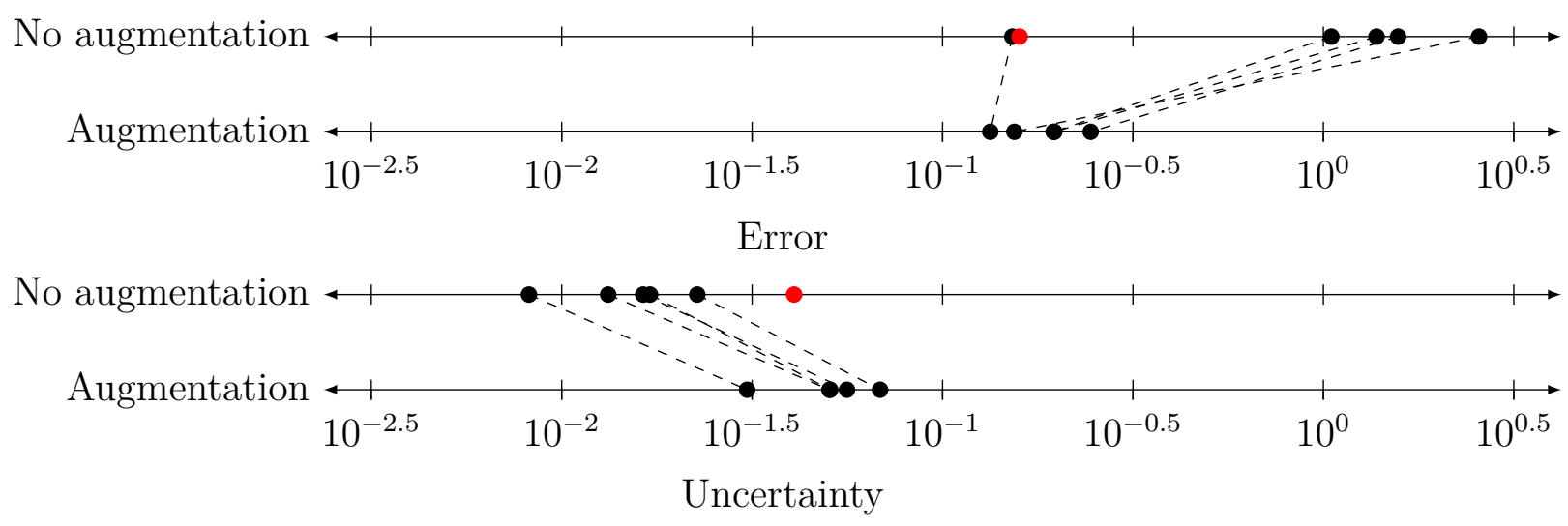

(b) Results spread. Black dots are $n_{\mathrm{en}}=12$ cases. Red dot is $n_{\mathrm{en}}=48$ case.

Figure 5.10: Statistically stationary flame - reproduction scenario augmentation results with small-ensemble ETKF

cost. This shows that, if a background set is well-matched to the augmented run under consideration, the augmented ensemble produced with distance-based classification is virtually indistinguishable from the equivalent large ensemble. Even with the more complicated test problem of statistically stationary reacting flow, augmentation is robust enough to perform just as well as it did with the model problems in Chapter 4.

For the extinction event, the performance is improved significantly from the unaugmented case, though the results overall are not as strong as for the statistically stationary flame. We see in Figure 5.11b that though the error spread is reduced from the unaugmented case, it still spans an order of magnitude. The error mean is lowered by nearly an order 


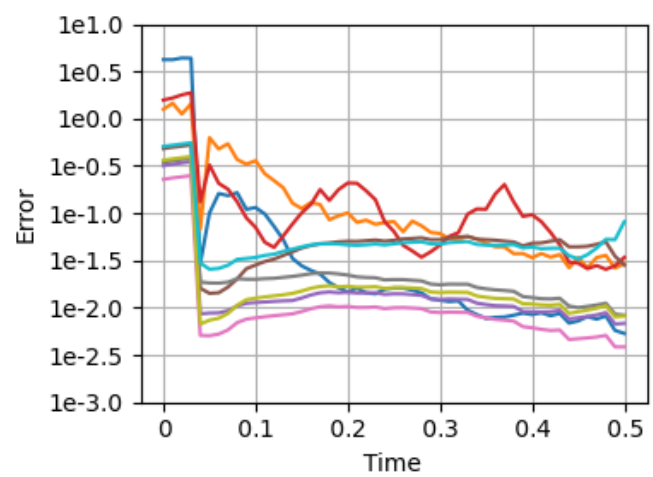

Error

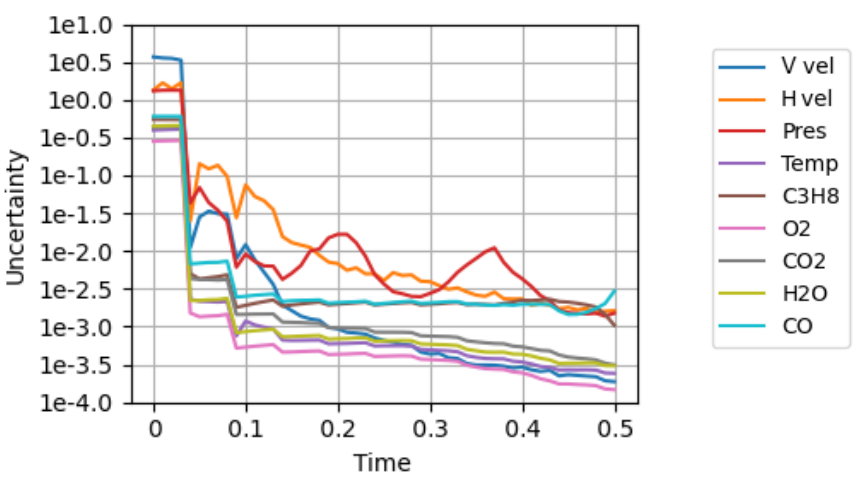

Uncertainty

(a) Example results with small-ensemble ETKF

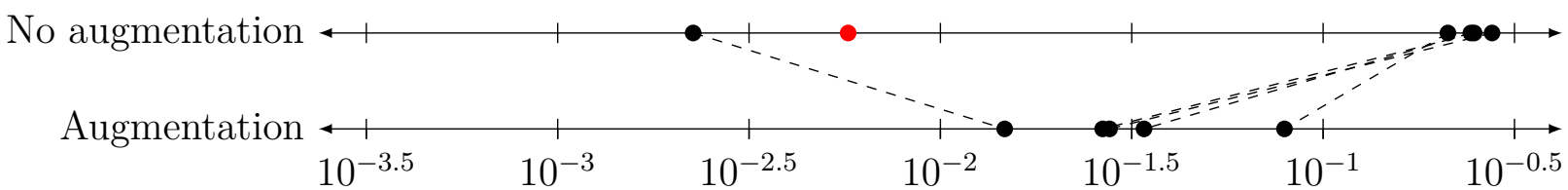

Error

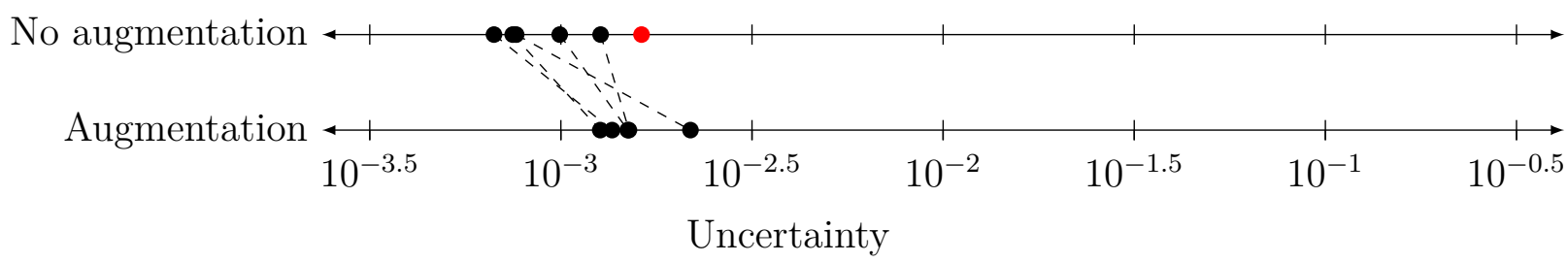

(b) Results spread. Black dots are $n_{\mathrm{en}}=12$ cases. Red dot is $n_{\mathrm{en}}=48$ case.

Figure 5.11: Extinction event - reproduction scenario augmentation results with smallensemble ETKF

of magnitude, bringing it to roughly $3 \%$ error. The uncertainty is more in line with the unaugmented filter of $n_{\mathrm{en}}=48$, however this is not a large increase from the unaugmented $n_{\mathrm{en}}=12$ uncertainty. This shows that the DA performance with reacting flow problems is not necessarily limited by an underestimated uncertainty, but by a limited ensemble basis, as with the model problems discussed in Chapter 4. Augmentation leads to significant performance improvements without a large increase to uncertainty. The ETKF does not however perform as well as in the case of the statistically stationary flame, likely due to the transient nature of the problem. We hypothesize that more generating runs may be required before the background set sufficiently captures most relevant statistical behaviour. 


\subsubsection{Different Trajectory}

For the different trajectory scenario, we only consider the statistically stationary flame case. As mentioned in Section 5.4, this is because we do not expect the background set generated from fully transient extinction event to generalize to a test case outside of the training data.

We consider the same background set that was generated for the reproduction scenario. This background set corresponds to the time interval $[0,0.5]$. We perform an augmented run that is offset forward in time by two observation periods, i.e., the time interval $[0.1,0.6]$. Recall from Figure 5.4 that this shift induces a significant change in the velocity field. An example run is shown in Figure 5.12a and the spread is shown in Figure 5.12b.

As discussed in Section 3.3, the background set depends on two aspects of the generating run: the ground truth trajectory, and the uncertainty in the forecast ensemble. By the second observation periods at $t=0.1$, we see in Figure 5.7 that the uncertainty has decreased almost to its minimum, as the ensemble has converged on a more accurate state estimate. An offset augmented run therefore has a background set that is well-matched in ground-truth trajectory but poorly-matched in uncertainty.

Regardless, we see a significant performance improvement in Figure 5.12b. Uncertainty has converged toward the large-ensemble level. Error is reduced significantly, however it remains slightly higher than the large-ensemble error. Augmentation leads to very consistent performance across the test cases, despite the mismatched uncertainty with the background set. It may be easy now to see how, if augmentation were to be applied to a real-world multiple-query problem involving reacting flow, a well-chosen training strategy would produce a background set that reduces computational cost for a majority of filter runs. 


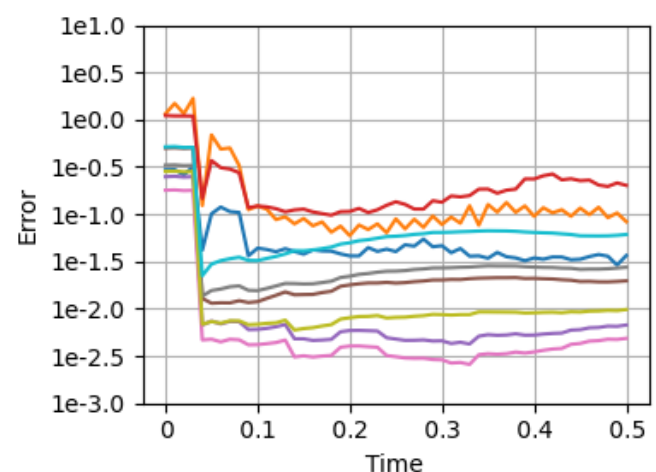

Error

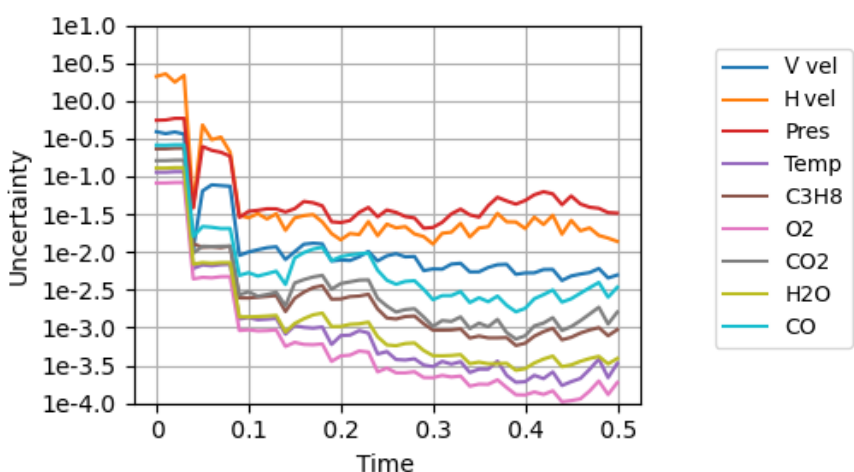

Uncertainty

(a) Example results with small-ensemble ETKF

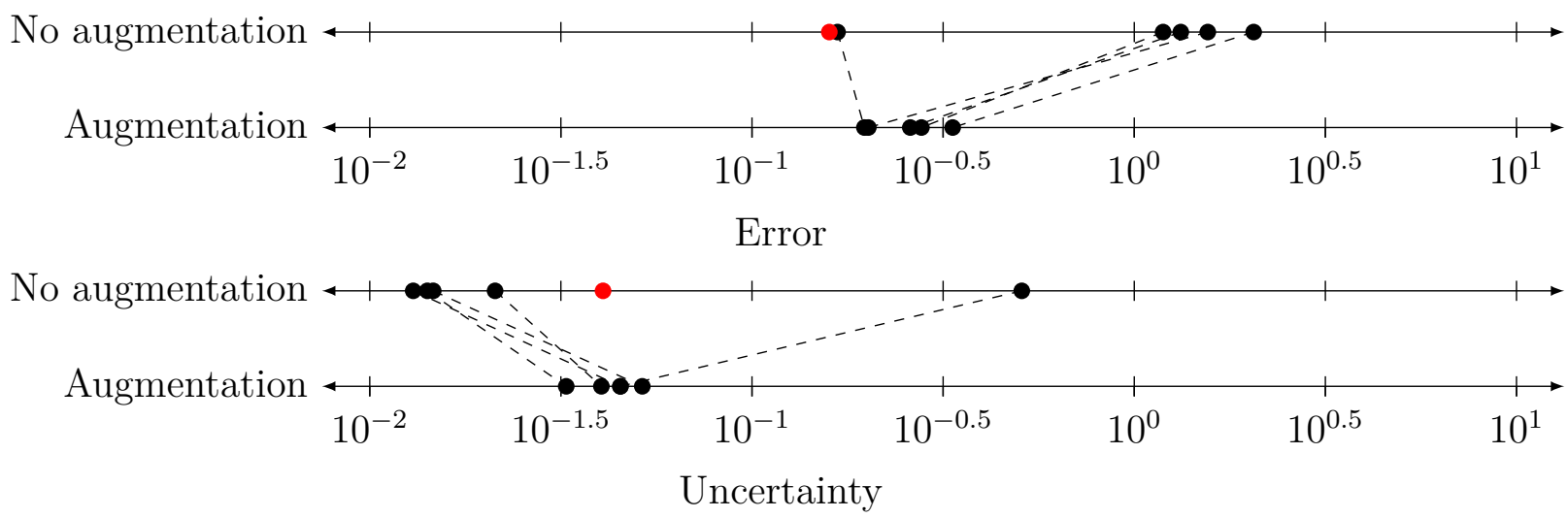

(b) Results spread. Black dots are $n_{\mathrm{en}}=12$ cases. Red dot is $n_{\mathrm{en}}=48$ case.

Figure 5.12: Statistically stationary flame - different trajectory augmentation results with small-ensemble ETKF 


\section{Chapter 6}

\section{Conclusion}

\subsection{Summary}

In this work we have demonstrated the technique of covariance augmentation, whereby we supplement the ensemble state used in a filter with artificial members drawn from background distributions. These backgrounds are generated from prior runs of the filter, reducing the computational cost of subsequent augmented runs by drawing upon a a library of background covariances that capture different statistical behaviours. We have shown this method to be effective with model problems and with two cases of reacting flow.

We have also demonstrated that the background set may be generally useful even in cases where the system trajectory is different or the governing equations are more chaotic than anticipated. The background set is not a perfect match to the statistical regimes of these scenarios, however the classification scheme is robust enough to choose the most appropriate background among imperfect options, and that background contains enough relevant information to improve performance significantly.

Assuming that the set of background distributions is well-matched to a particular augmented run, there are still two potential sources of error. The first is the classification scheme, which may not be capable of matching the under-resolved forecast ensemble to the most appropriate background distribution. The second is the level of background clustering, which risks producing a under-sized set of over-smoothed background distributions, diluting the nuances of particular statistical regimes. The reproduction scenario tests we performed show that both of these concerns are manageable. The classification scheme is able to robustly select the correct background among a well-matched set, as evidenced by the low mean error, the well-resolved uncertainty level, and the extremely consistent performance in all reproduction scenario tests. The application of background clustering did not compromise augmentation performance and may be modified to a level appropriate for the system 
under consideration.

We have shown in theoretical terms how augmentation may be applied to speed up computation of multiple-query problems. If a plan calls for $n_{f}$ runs of the filter, a carefully chosen subset of those runs may be used as generating runs, reducing the computational cost significantly for the remaining augmented runs. This relies on the empirical observation that the ensemble size required to classify an ensemble among statistical flow regimes is much smaller than the ensemble size required for an ensemble to resolve the covariance by itself, though we do not know in general how much smaller we can expect it to be.

\subsection{Limitations and Future Work}

The limitations of this thesis, and in particular our realization of covariance augmentation, are summarized here. We also discuss where these limitations lead to opportunities for future work.

\section{Background Set Memory Requirements}

In this work, we took a straightforward approach to constructing the background covariance matrices. The forecast ensembles from statistically-resolved runs are stored and clustered into background ensembles. A metric for distance between probability distributions is used to match the under-resolved natural forecast ensemble to the appropriate background. Artificial members are then drawn from the background, supplementing the natural ensemble. This approach is enough to prove the concept, but the straightforward storage and reference of forecast ensembles as backgrounds could be made far more memory-efficient. For example, techniques from reduced order modelling could be incorporated to represent all backgrounds using a reduced basis.

For backgrounds with significant clustering, storage space may also be minimized with lossy compression techniques, such as a truncated SVD representation. Once this is done though, the background set can no longer be grown with subsequent generating runs, therefore it should only be done when the background set is considered sufficiently comprehensive.

\section{Background Parameterization}


As discussed in Chapter 3, the statistical regime classification problem posed by background generation is distinct from simple flow regime classification. This is because each forecast ensemble is dependent on both the trajectory of the model and the uncertainty, which in turn is dependent on the initial conditions and observation model. How the shape of a background distribution changes with uncertainty is not well-understood. If it is possible to go from a purely data-driven approach to a partially parameterized one, such as parameterizing the background covariance using uncertainty in the forecast ensemble, this may reduce the size of the background set.

\section{Level of Background Clustering}

The number of background clusters $n_{B}$ was not given significant focus in this work. This was for two reasons. First, at least for the problems we considered, it is relatively unimportant in determining the performance of augmented runs, as shown in Section 4.5.1. Second, because our most complicated demonstration case was a relatively simple reacting flow problem, memory constraints were not a significant concern. For practical problems however, it will be worth examining the tradeoffs in different values of $n_{B}$ more closely. For simple problems like ours, our results suggest it is preferable to minimize clustering and rely on the classification scheme to find the appropriate, highly-specific background. The memory demands of such an approach prevent it from being scaled easily to larger problems. This is due to both the larger forecast ensemble matrices produced by the generating runs, and the greater number of background ensembles necessary to capture all flow regimes. To minimize the number of background ensembles, clustering ought to be used judiciously to allow augmentation to be applied to larger problems while not overly compromising the specificity of background covariances.

\section{Bayesian Classification}

Bayesian classification may perform well in circumstances where the background set is well-matched to the augmented run, but it is not robust to imperfect background sets, as was shown with high levels of clustering. We believe this method may eventually prove useful for very high-dimensional problems because, when the covariance rank is much larger than the number of samples, distance-based classification becomes more prone to falsely selecting

undersized background covariances. In its current form however, Bayesian classification tends to have the opposite bias, leading to diverging solutions. 


\section{Generating Runs with Artificial Resolving Techniques}

There are numerous artificial resolving techniques within the categories of inflation and localization that were not discussed in this work. These techniques are not only useful to compare with the performance of augmentation, but also to utilize in generating runs. The model problems presented here highlighted the deficiencies of these techniques, but where these techniques are well-suited to the problem under consideration, the modified forecast ensembles could be used to populate the background set at significantly lower computational cost than running an unmodified large-ensemble filter.

\section{Localized Augmentation}

Reacting flows introduce not only multiple length scales as discussed previously, but multiple relevant timescales which govern different areas of the flow and even different variables. For example, disparate fluid dynamic and chemical timescales could cause either velocity or species fractions to be the dominant sources of error, as we saw in our reacting flow example in Chapter 5. A localized augmentation scheme could be used in such cases to resolve more difficult areas of the flow or more difficult flow variables without affecting most of the ensemble, reducing computational cost further.

\section{Training Strategies}

Augmentation is a form of machine learning. We train our background set using very simple test cases in order to prove the concept, however in future more formal study ought to be devoted to the design of training strategies.

Though we tested our augmentation scheme using reacting flow in the reproduction and different trajectory scenarios, there are more cases of practical interest that warrant examination. Using a case of statistically stationary behaviour, such as the diffusion flame considered in Chapter 5, future research should examine augmentation training strategies in a model regime where it is potentially achievable for the background set to be comprehensive of flow behaviour. This would also allow us to determine a reasonable level of best-case performance for augmentation outside of the reproduction scenario.

\section{Reacting Flow Scenarios}

Due to the computational effort required, we were limited in the level of analysis we 
could perform in the cases of reacting flow. We were able to provide a proof-of-concept for augmentation in reacting flow problems, however future research should further characterize its performance in more practical scenarios, especially where model limitations become significant. For example, we did not consider highly turbulent flows, where the limitations of LES would be most apparent.

Testing with synthetic problems allows us to perform a controlled comparison between DA techniques; however, to evaluate their performance in a more absolute sense, future work should examine a case using real-world experimental data. This would capture the effects of model deficiencies and more realistic experimental setups, which would almost certainly lead to a more gradual convergence in error and uncertainty over time than in our reacting flow case. It would also require the construction of a more realistic observation noise model than the simple Gaussian one we assumed. 


\section{Appendix A}

\section{Ensemble DA Implementation}

The ensemble DA formulation in Chapter 2 and augmentation in Chapter 3 require at several points that we calculate a forecast covariance $\mathbf{C}_{k \mid k-1}$ or a background covariance $\mathbf{B}$. The memory demands of storing such a covariance matrix in a naive manner scale with $O\left(n_{u}^{2}\right)$. For example, the reacting flow simulation considered in Chapter 5 had a state size of $n_{u}=$ 238820. Assuming each element of the covariance matrix is a double-precision floating point number, i.e., requires 8 bytes, a $238820 \times 238820$ covariance matrix requires 456 GB of space. Even this is relatively small compared to the state sizes in practical DA systems [15], so we require reformulations of our techniques that do not explicitly form large covariance matrices.

\section{A.1 Ensemble DA}

The only step of the ensemble Kalman filter or ensemble transform Kalman filter that requires forming the forecast covariance is the calculation of the Kalman gain using a linear observation model in (2.13). This equation is only presented for the sake of illustration; the more general form presented in (2.12) should always be used, as it does not require forming any matrix larger than the Kalman gain matrix. If using a linear observation model $\mathbf{H}$, as we do in this work, then we can expand the observation ensemble $\tilde{\mathbf{V}}_{k \mid k-1}$ and covariance $\mathbf{D}_{k \mid k-1}$ to obtain

$$
\mathbf{K}_{k}=\frac{1}{n_{\mathrm{en}}-1} \tilde{\mathbf{U}}_{k \mid k-1}\left(\mathbf{H} \tilde{\mathbf{U}}_{k \mid k-1}\right)^{T}\left(\frac{1}{n_{\mathrm{en}}-1}\left(\mathbf{H} \tilde{\mathbf{U}}_{k \mid k-1}\right)\left(\mathbf{H} \tilde{\mathbf{U}}_{k \mid k-1}\right)^{T}+\mathbf{R}\right)^{-1}
$$

to substitute for (2.13) in practice. The Kalman identity (2.14) also appears to require forming the forecast covariance, however this is also presented merely for illustration. When the ETKF is used, the analysis step automatically produces an analysis ensemble $\mathbf{U}_{k \mid k}$ whose corresponding covariance $\mathbf{C}_{k \mid k}$ satisfies the Kalman identity. 


\section{A.2 Augmentation}

There are two steps of covariance augmentation where the background covariance $\mathbf{B}$ appears to be explicitly formed. The first is in the generation of artificial members from a steady background distribution according to (3.4). A common method [9] to draw random values from a multivariate normal distribution with mean $\overline{\mathbf{u}}_{k \mid k-1}$ and covariance $\mathbf{B}$ is:

1. Form $\hat{\mathbf{W}}=\frac{1}{\sqrt{n_{\mathrm{en} B}-1}} \tilde{\mathbf{W}}$. Note that $\mathbf{B}=\hat{\mathbf{W}} \hat{\mathbf{W}}^{T}$.

2. Generate $\mathbf{z}=\left[z_{1}, \ldots, z_{n_{u}}\right]^{T}$ where $\left\{z_{1}, \ldots, z_{n_{u}}\right\}$ are drawn from a standard normal distribution.

3. Form the artificial member $\mathbf{u}_{\mathrm{art}}=\overline{\mathbf{u}}_{k \mid k-1}+\hat{\mathbf{W}} \mathbf{z}$.

This method is repeated until we have the desired quantity of artificial members.

The second is in calculating the Wasserstein distance metric between forecast covariance $\mathbf{C}_{k \mid k-1}$ and background $\mathbf{B}$ as per (3.6). We start in a similar manner to the artificial member generation above, by forming $\hat{\mathbf{U}}_{k \mid k-1}=\frac{1}{\sqrt{n_{\mathrm{en}}-1}} \tilde{\mathbf{U}}_{k \mid k-1}$ and $\hat{\mathbf{W}}=\frac{1}{\sqrt{n_{\mathrm{en} B}-1}} \tilde{\mathbf{W}}$. We then take the singular value decomposition of $\hat{\mathbf{U}}_{k \mid k-1}$ and $\hat{\mathbf{W}}$, such that the corresponding covariance matrices can be expressed as

$$
\begin{gathered}
\mathbf{B}=\mathbf{Q}_{\mathbf{B}} \mathbf{S}_{\mathbf{B}}^{2} \mathbf{Q}_{\mathbf{B}}^{T} \\
\mathbf{C}_{k \mid k-1}=\mathbf{Q}_{\mathbf{C}} \mathbf{S}_{\mathbf{C}}^{2} \mathbf{Q}_{\mathbf{C}}^{T},
\end{gathered}
$$

where $\mathbf{Q}_{\mathbf{B}}$ and $\mathbf{Q}_{\mathbf{C}}$ are unitary matrices and $\mathbf{S}_{\mathbf{B}}$ and $\mathbf{S}_{\mathbf{C}}$ are diagonal matrices of singular values. When we insert these into (3.6), we obtain

$$
\begin{aligned}
d^{2} & =\operatorname{tr}\left(\mathbf{Q}_{\mathbf{C}} \mathbf{S}_{\mathbf{C}}^{2} \mathbf{Q}_{\mathbf{C}}^{T}+\mathbf{Q}_{\mathbf{B}} \mathbf{S}_{\mathbf{B}}^{2} \mathbf{Q}_{\mathbf{B}}^{T}-2\left(\left(\mathbf{Q}_{\mathbf{C}} \mathbf{S}_{\mathbf{C}}^{2} \mathbf{Q}_{\mathbf{C}}^{T}\right)^{1 / 2} \mathbf{Q}_{\mathbf{B}} \mathbf{S}_{\mathbf{B}}^{2} \mathbf{Q}_{\mathbf{B}}^{T}\left(\mathbf{Q}_{\mathbf{C}} \mathbf{S}_{\mathbf{C}}^{2} \mathbf{Q}_{\mathbf{C}}^{T}\right)^{1 / 2}\right)^{1 / 2}\right) \\
& =\sum_{j=1}^{n_{\mathrm{en}}-1} \varsigma_{j}^{2}\left(\hat{\mathbf{U}}_{k \mid k-1}\right)+\sum_{j=1}^{n_{\mathrm{en} B}-1} \varsigma_{j}^{2}(\hat{\mathbf{W}})-\operatorname{tr}\left(2\left(\mathbf{Q}_{\mathbf{C}} \mathbf{S}_{\mathbf{C}} \mathbf{Q}_{\mathbf{C}}^{T} \mathbf{Q}_{\mathbf{B}} \mathbf{S}_{\mathbf{B}}^{2} \mathbf{Q}_{\mathbf{B}}^{T} \mathbf{Q}_{\mathbf{C}} \mathbf{S}_{\mathbf{C}} \mathbf{Q}_{\mathbf{C}}^{T}\right)^{1 / 2}\right) \\
& =\sum_{j=1}^{n_{\mathrm{en}}-1} \varsigma_{j}^{2}\left(\hat{\mathbf{U}}_{k \mid k-1}\right)+\sum_{j=1}^{n_{\mathrm{en} B}-1} \varsigma_{j}^{2}(\hat{\mathbf{W}})-\operatorname{tr}\left(2\left(\mathbf{S}_{\mathbf{C}} \mathbf{Q}_{\mathbf{C}}^{T} \mathbf{Q}_{\mathbf{B}} \mathbf{S}_{\mathbf{B}}^{2} \mathbf{Q}_{\mathbf{B}}^{T} \mathbf{Q}_{\mathbf{C}} \mathbf{S}_{\mathbf{C}}\right)^{1 / 2}\right) \\
& =\sum_{j=1}^{n_{\mathrm{en}}-1} \varsigma_{j}^{2}\left(\hat{\mathbf{U}}_{k \mid k-1}\right)+\sum_{j=1}^{n_{\mathrm{en} B}-1} \varsigma_{j}^{2}(\hat{\mathbf{W}})-2 \sum_{j=1}^{n_{\mathrm{en}}-1} \varsigma_{j}\left(\mathbf{S}_{\mathbf{C}} \mathbf{Q}_{\mathbf{C}}^{T} \mathbf{Q}_{\mathbf{B}} \mathbf{S}_{\mathbf{B}}\right)
\end{aligned}
$$

where $\varsigma_{j}\left(\hat{\mathbf{U}}_{k \mid k-1}\right)$ is the $j$ th singular value of $\hat{\mathbf{U}}_{k \mid k-1}, \varsigma_{j}(\hat{\mathbf{W}})$ is the $j$ th singular value of $\hat{\mathbf{W}}$, and $\varsigma_{j}\left(\mathbf{S}_{\mathbf{C}} \mathbf{Q}_{\mathbf{C}}^{T} \mathbf{Q}_{\mathbf{B}} \mathbf{S}_{\mathbf{B}}\right)$ is the $j$ th singular value of the cross-multiplied term $\mathbf{S}_{\mathbf{C}} \mathbf{Q}_{\mathbf{C}}^{T} \mathbf{Q}_{\mathbf{B}} \mathbf{S}_{\mathbf{B}}$. 


\section{Appendix B}

\section{CFD Interfacing}

Raptor, like most CFD tools available, is designed as a standalone reacting flow solver, not for use in DA [25]. Using it as a process model for DA as per (2.1) requires that we execute the code for very short time windows $\Delta t=t_{k}-t_{k-1}$, that we provide an initial state $\mathbf{u}_{k-1}$, and that we obtain the propagated state $\mathbf{u}_{k}$. To accomplish this, we developed a wrapper that interfaces between Raptor and the DA machinery.

As with many CFD codes [10], the spatial domain is partitioned into a number of "blocks" for parallelization each of which is assigned to a separate process. For the reacting flow setup we consider in Chapter 5, the spatial domain consists of 80 blocks. Consequently, the flow state is stored as a list of all 80 blocks. This is the format Raptor consistently uses for input and output. Each block is a 4-D array where the axes are flow variable $q, x$ mesh index, $y$ mesh index, and $z$ mesh index.

DA on the other hand operates in terms of state vectors, requiring us to convert to and from the flow state used in CFD. To convert each flow variable component of a block to a state vector $\mathbf{u}$, we flatten it according to

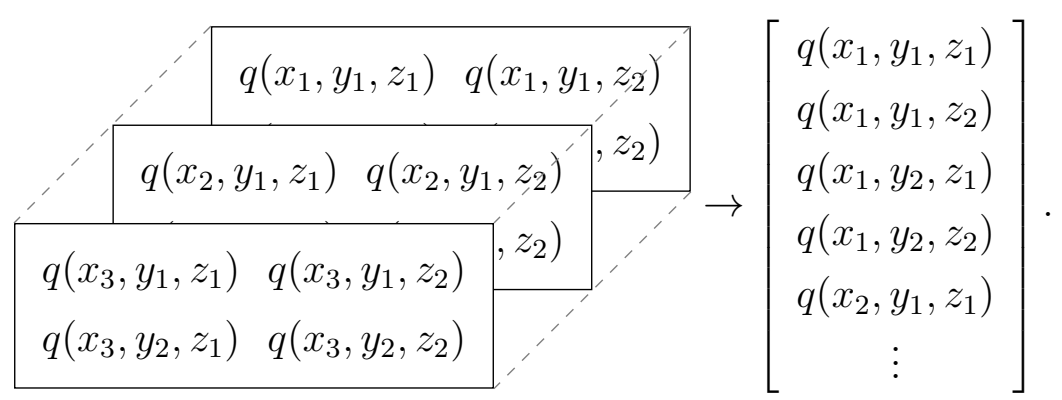

We concatenate the flow-variable-specific state vectors to produce a block-specific state vector, then concatenate all block-specific state vectors to produce an comprehensive state vector u. When we want to evaluate Raptor from a certain initial state, we reverse this process to convert a state vector back into a list of blocks that Raptor can take as input. 


\section{Bibliography}

[1] A. Abou-Taouk, S. Sadasivuni, D. Lörstad, and L. Eriksson. "Evaluation of Global Mechanisms for LES Analysis of SGT-100 DLE Combustion System". In: vol. Volume 1B: Combustion, Fuels and Emissions. Turbo Expo: Power for Land, Sea, and Air. June 2013. DOI: 10.1115/GT2013-95454.

[2] S. E. Ahmed, S. Pawar, and O. San. "PyDA: A Hands-On Introduction to Dynamical Data Assimilation with Python". In: Fluids 5.4 (2020). ISSN: 2311-5521. DOI: 10.3390/ fluids5040225.

[3] J. L. Anderson. "An adaptive covariance inflation error correction algorithm for ensemble filters". In: Tellus A 59.2 (2007), pp. 210-224. DOI: 10.1111/j.1600-0870. $2006.00216 . x$.

[4] J. L. Anderson. "Localization and Sampling Error Correction in Ensemble Kalman Filter Data Assimilation". In: Monthly Weather Review 140.7 (2012), pp. 2359-2371. DOI: $10.1175 /$ MWR-D-11-00013.1.

[5] M. Bruchhausen, F. Guillard, and F. Lemoine. "Instantaneous measurement of twodimensional temperature distributions by means of two-color planar laser induced fluorescence (PLIF)". In: Experiments in Fluids 38.1 (Jan. 2005), pp. 123-131. ISSN: 1432-1114. DOI: $10.1007 / \mathrm{s} 00348-004-0911-2$.

[6] G. Evensen. Data assimilation: the ensemble Kalman filter. Springer, 2009. DOI: 10. 1007/978-3-642-03711-5.

[7] M. Fisher. Assimilation techniques (3): 3dVar. European Centre for Medium-Range Weather Forecasts (ECMWF), 2002.

[8] M. Fisher. Assimilation Techniques (4): 4dVar. European Centre for Medium-Range Weather Forecasts (ECMWF), 2002.

[9] J. Gentle. Computational Statistics. Jan. 2009. IsBn: 978-0-387-98143-7. DOI: 10.1007/ 978-0-387-98144-4. 
[10] L.Y.M. Gicquel, N. Gourdain, J.-F. Boussuge, H. Deniau, G. Staffelbach, P. Wolf, and T. Poinsot. "High performance parallel computing of flows in complex geometries". In: Comptes Rendus Mecanique 339.2 (2011). High Performance Computing, pp. 104-124. ISSN: 1631-0721. DOI: https://doi.org/10.1016/j.crme.2010.11.006.

[11] L.Y.M. Gicquel, G. Staffelbach, and T. Poinsot. "Large Eddy Simulations of gaseous flames in gas turbine combustion chambers". In: Progress in Energy and Combustion Science 38.6 (2012), pp. 782-817. ISSN: 0360-1285. DOI: https://doi.org/10.1016/ j.pecs. 2012.04 .004 .

[12] S. J. Greybush, E. Kalnay, T. Miyoshi, K. Ide, and B. R. Hunt. "Balance and Ensemble Kalman Filter Localization Techniques". In: Monthly Weather Review 139.2 (Feb. 2011), pp. 511-522. DOI: 10.1175/2010MWR3328.1.

[13] S. W. Grib, N. Jiang, P. S. Hsu, P. M. Danehy, and S. Roy. "Rayleigh-scattering-based two-dimensional temperature measurement at $100-\mathrm{kHz}$ frequency in a reacting flow". In: Optics Express 27.20 (Sept. 2019), pp. 27902-27916. DOI: 10.1364/OE.27.027902.

[14] T.M. Hamill. "Ensemble-based data assimilation." In: Seminar on Predictability of weather and climate, 9-13 September 2002. ECMWF. Shinfield Park, Reading: ECMWF, 2003, pp. 83-112.

[15] P. Houtekamer and F. Zhang. "Review of the ensemble Kalman filter for atmospheric data assimilation". In: Monthly Weather Review 144.12 (Dec. 2016), pp. 4489-4532. DOI: $10.1175 / \mathrm{mwr}-\mathrm{d}-15-0440.1$.

[16] R. Johnson, H. Wu, and M. Ihme. "A general probabilistic approach for the quantitative assessment of LES combustion models". In: Combustion and Flame 183 (Sept. 2017), pp. 88-101. ISSN: 0010-2180. DOI: 10.1016/j.combustflame.2017.05.004.

[17] Y. Kuramoto. "Diffusion-Induced Chaos in Reaction Systems". In: Progress of Theoretical Physics Supplement 64 (Feb. 1978), pp. 346-367. ISSN: 0375-9687. DOI: 10. 1143/PTPS . 64.346.

[18] J. Labahn, H. Wu, B. Coriton, J. Frank, and M. Ihme. "Data assimilation using highspeed measurements and LES to examine local extinction events in turbulent flames". In: Proceedings of the Combustion Institute 37.2 (2019), pp. 2259-2266. DOI: 10.1016/ j.proci.2018.06.043.

[19] J. W. Labahn, H. Wu, S. R. Harris, B. Coriton, J. H. Frank, and M. Ihme. "Ensemble Kalman Filter for Assimilating Experimental Data into Large-Eddy Simulations of Turbulent Flows". In: Flow, Turbulence and Combustion (2019). ISSN: 1573-1987. DOI: $10.1007 / \mathrm{s} 10494-019-00093-1$. 
[20] K. Law, A. Stuart, and K. Zygalakis. Data Assimilation. Springer International Publishing, 2015. DOI: 10.1007/978-3-319-20325-6.

[21] L. Lei, J. Anderson, and G. Romine. "Empirical Localization Functions for Ensemble Kalman Filter Data Assimilation in Regions with and without Precipitation". In: Monthly Weather Review 143 (May 2015), p. 150522112937007. DOI: 10.1175/MWR-D14-00415.1.

[22] E. N. Lorenz. "Designing chaotic models". In: Journal of the atmospheric sciences 62.5 (2005), pp. 1574-1587. ISSN: 0022-4928. DOI: 10.1175/JAS3430.1.

[23] E. N. Lorenz. "Predictability: a problem partly solved". In: Seminar on Predictability, 4-8 September 1995. Vol. 1. ECMWF. Shinfield Park, Reading: ECMWF, 1995, pp. 118.

[24] K. Nakamura, N. Hirose, B. Choi, and T. Higuchi. "Particle Filtering in Data Assimilation and Its Application to Estimation of Boundary Condition of Tsunami Simulation Model". In: Jan. 2009, pp. 353-366. ISBN: 978-3-540-71055-4. DOI: 10.1007/978-3540-71056-1_19.

[25] J. C. Oefelein. "Advances in Modeling Supercritical Fluid Behavior and Combustion in High-Pressure Propulsion Systems". In: AIAA Science and Technology Forum and Exposition. 2019. DOI: 10.2514/6.2019-0634.

[26] D. F. Parrish and J. C. Derber. "The National Meteorological Center's Spectral StatisticalInterpolation Analysis System". In: Monthly Weather Review 120.8 (1992), pp. 17471763. DOI: $10.1175 / 1520-0493(1992) 120<1747$ : TNMCSS $>2$. 0. CO $; 2$.

[27] T. Poinsot. "Prediction and control of combustion instabilities in real engines". In: Proceedings of the Combustion Institute 36.1 (2017), pp. 1-28. ISSN: 1540-7489. DOI: https://doi.org/10.1016/j.proci.2016.05.007.

[28] S. B. Pope. "Small scales, many species and the manifold challenges of turbulent combustion". In: Proceedings of the Combustion Institute 34.1 (Jan. 2013), pp. 1-31. DOI: 10.1016/j.proci.2012.09.009.

[29] F. Rabier and Z. Liu. "Variational data assimiltion: theory and overview". In: Seminar on Recent developments in data assimilation for atmosphere and ocean, 8-12 September 2003. European Centre for Medium-Range Weather Forecasts (ECMWF). Shinfield Park, Reading: ECMWF, 2003, pp. 29-44.

[30] T. Rippl, A. Munk, and A. Sturm. "Limit laws of the empirical Wasserstein distance: Gaussian distributions". In: Journal of Multivariate Analysis 151 (July 2015). DOI: 10.1016/j.jmva.2016.06.005. 
[31] Z. Shen, Y. Tang, X. Li, and Y. Gao. "On the Localization in Strongly Coupled Ensemble Data Assimilation Using a Two-Scale Lorenz Model". In: Earth and Space Science 8.3 (2021), e2020EA001465. DOI: https://doi.org/10.1029/2020EA001465.

[32] G. Sivashinsky. "Nonlinear analysis of hydrodynamic instability in laminar flames-I. Derivation of basic equations". In: Acta Astronautica 4.11 (1977), pp. 1177-1206. ISSN: 0094-5765. DOI: https://doi.org/10.1016/0094-5765(77)90096-0.

[33] A. Talamelli, F. Persiani, J. Fransson, P. Alfredsson, A. Johansson, H. Nagib, J. Rueedi, K. Sreenivasan, and P. Monkewitz. "CICLoPE - A response to the need for high Reynolds number experiments". In: Fluid Dynamics Research 41 (Apr. 2009). DOI: $10.1088 / 0169-5983 / 41 / 2 / 021407$.

[34] P.G. Tucker. "Trends in turbomachinery turbulence treatments". In: Progress in Aerospace Sciences 63 (2013), pp. 1-32. ISSN: 0376-0421. DOI: https://doi.org/10.1016/j. paerosci.2013.06.001.

[35] B. Uzunoglu, S. J. Fletcher, M. Zupanski, and I. M. Navon. "Adaptive ensemble reduction and inflation". In: Quarterly Journal of the Royal Meteorological Society 133.626 (2007), pp. 1281-1294. DOI: https://doi.org/10.1002/qj.96.

[36] B. Wang, J. Liu, L. Liu, S. Xu, and W. Huang. "An approach to localization for ensemble-based data assimilation". In: PLOS ONE 13.1 (Jan. 2018), pp. 1-23. DOI: 10.1371/journal . pone. 0191088 .

[37] Y. Ying, F. Zhang, and J. L. Anderson. "On the Selection of Localization Radius in Ensemble Filtering for Multiscale Quasigeostrophic Dynamics". In: Monthly Weather Review 146.2 (2018), pp. 543-560. DOI: 10.1175/MWR-D-17-0336.1.

[38] H. Yu, T. Jaravel, M. Ihme, M. P. Juniper, and L. Magri. "Data Assimilation and Optimal Calibration in Nonlinear Models of Flame Dynamics". In: Journal of Engineering for Gas Turbines and Power 141.12 (Nov. 2019). 121010. ISSN: 0742-4795. DOI: $10.1115 / 1.4044378$.

[39] H. Yu, M. P. Juniper, and L. Magri. "A data-driven kinematic model of a ducted premixed flame". In: Proceedings of the Combustion Institute (2020). ISSN: 1540-7489. DOI: https://doi.org/10.1016/j.proci.2020.06.137. 